\title{
A Distributed Approach to Underwater Acoustic Communications
}

by

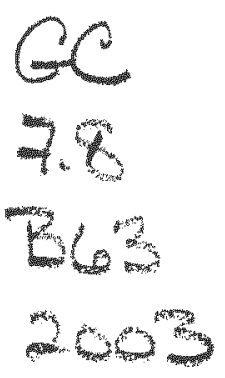

Christopher George Bohner

Submitted to the Department of Ocean Engineering in partial fulfillment of the requirements for the degree of

Master of Science in Ocean Engineering at the

MASSACHUSETTS INSTITUTE OF TECHNOLOGY and the

\section{WOODS HOLE OCEANOGRAPHIC INSTITUTION}

September 2003

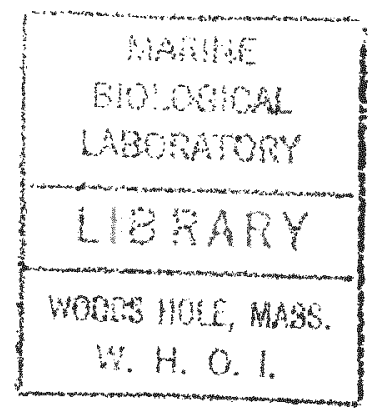

(C) 2003 Christopher George Bohner. All rights reserved.

The author hereby grants to MIT and WHOI permission to reproduce and distribute publicly paper and electronic copies of this thesis document in whole or in part.

Author .

Joint Program in Applied Ocean Science and Engineering Massachusetts Institute of Technology And Woods Hole Oceanographic Institution

September 2003

Certified by.

Professor Arthur B. Baggeroer Ford Professor of Engineering Secretary of the Navy/Chief of Naval Operations Chair for Ocean Sciences Massachusetts Institute of Technology Th@ssis Supervisor

Accepted by $\ldots \ldots \ldots$.

Mark Grosenbaugh

Chairman, Joint Committee for Applied Ocean Science and Engineering Massachusetts Institute of Technology/Woods Hole Oceanographic Institution 


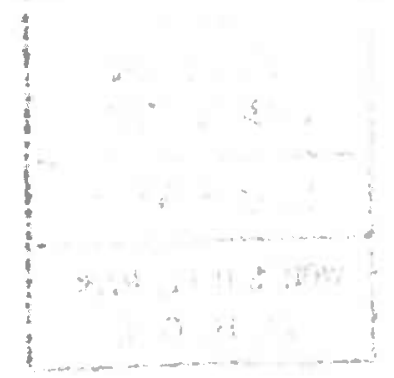




\title{
A Distributed Approach to Underwater Acoustic Communications
}

\author{
by \\ Christopher George Bohner \\ Submitted on July 15,2003 , in partial fulfillment \\ of the requirements for the degree of \\ Master of Science in Ocean Engineering \\ at the \\ MASSACHUSETTS INSTITUTE OF TECHNOLOGY \\ and the \\ WOODS HOLE OCEANOGRAPHIC INSTITUTION \\ July 2003
}

\begin{abstract}
A novel distributed underwater acoustic networking (UAN) protocol suitable for adhoc deployments of both stationary and mobile nodes dispersed across a relatively wide coverage area is presented. Nodes are dynamically clustered in a distributed manner based on the estimated position of one-hop neighbor nodes within a shallow water environment. The spatial dynamic cellular clustering scheme allows scalable communication resource allocation and channel reuse similar in design to land-based cellular architectures, except devoid of the need for a centralized controlling infrastructure. Simulation results demonstrate that relatively high degrees of interference immunity, network connectivity, and network stability can be achieved despite the severe limitations of the underwater acoustic channel.
\end{abstract}

Thesis Supervisor: Arthur B. Baggeroer

Title: Ford Professor of Engineering

Secretary of the Navy/Chief of Naval Operations Chair for Ocean Sciences 


\section{Acknowledgements}

My most gracious thanks are extended to my thesis advisor, Arthur Baggeroer, for his professional involvement in my academic career at both MIT and WHOI. His wise guidance and kind support were invaluable to my thesis work and instrumental to my academic success.

A special thanks to Milica Stojanovic for her personal influences and professional advice with regards to undersea communications. Her encouragement and support were important in the laying the foundation of my work.

My opportunity to participate in graduate studies at MIT and WHOI was made possible through the Secretary of the Navy's Program in Oceanography and Oceanographic Engineering and RADM Wilson, USN, Oceanographer of the Navy. A special thanks to Capt Ferdinand Diemer, USN, for his professional mentoring and personal advice throughout my naval career. His personal involvement allowed me to see and do things with my life I never thought possible.

While working hard at school, playing hard was made all the merrier by the multitude of friends I have made while at MIT and WHOI: Ted Schroeder, Rob Crofoot, Steven Roe, Doug Lemon, and Dan. Thanks for all the good times.

Finally, they say that behind every good man is an even greater woman. I am truly blessed to share my life with a woman whose personal attributes far exceed the depths of this assertion. The heavy burdens of my academic studies and professional endeavors were equally taxing to both my wife Shelly and my two children, Emily and Ryan. Their infinite patience and unwavering support were essential to my success as a student and serves as a testament to our love as a family. Thank you Shelly, I love you. 


\section{Table of Contents}

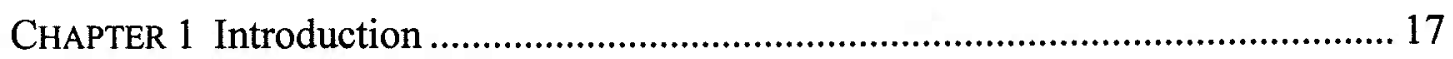

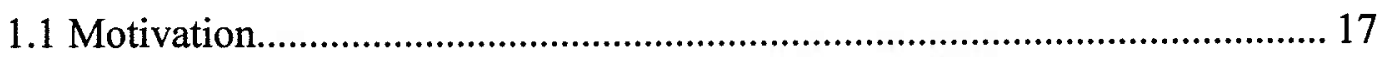

1.1.1 Centralized Communication Networks.................................................... 19

1.1.2 Distributed Communication Networks ................................................... 21

1.2 Complexities of Underwater Acoustic Network Design ................................. 23

1.3 Spatially Dynamic Cellular Clustering .......................................................... 25

1.4 Related Work ........................................................................................ 27

1.4.1 Adaptive Clustering for Ad-hoc Networks............................................ 28

1.4.2 Current Underwater Acoustic Network Designs .................................... 29

1.4.3 Spread-Spectrum Acoustic Modems ...................................................... 31

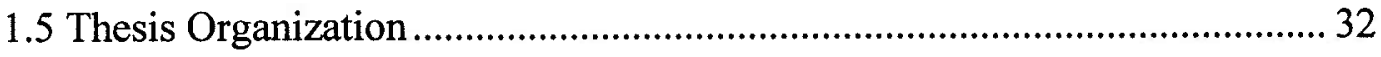

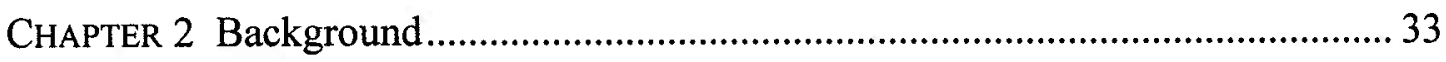

2.1 Underwater Acoustic Communications ........................................................ 33

2.1.1 Attenuation Losses............................................................................... 34

2.1.2 Geometric Spreading Losses .............................................................. 36

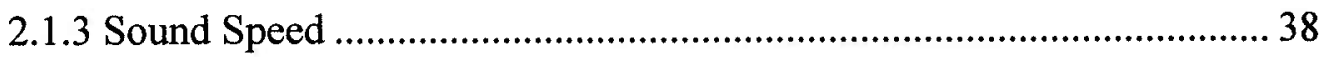

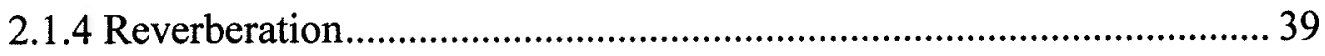

2.2 Spread Spectrum Communications................................................................ 42

2.3 The Cellular Concept ......................................................................................... 49

2.3.1 Multiple Access Techniques in Cellular Systems ................................ 50

2.3.2 Frequency Reuse............................................................................... 52

CHAPTER 3 Spatially Dynamic Cellular Clustering ....................................................5 56

3.1 Network Organization ............................................................................... 56

3.1.1 Determination of Network Structure …............................................... 57

3.1.2 Cell Coverage Area Considerations........................................................ 59

3.1.3 Node Clustering and Cell Orientation .................................................... 63

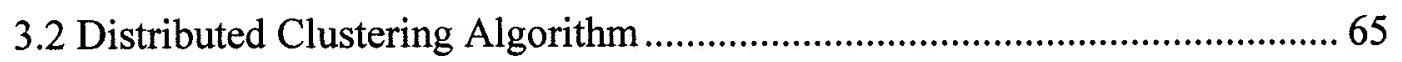




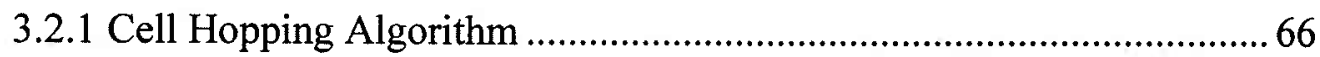

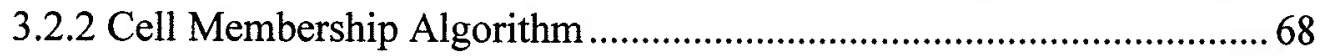

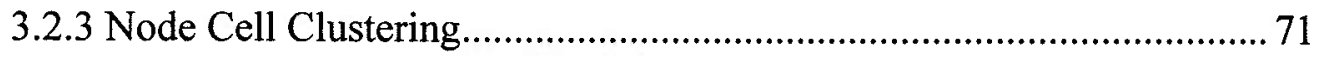

3.3 Medium Access Control Protocol ..................................................................... 76

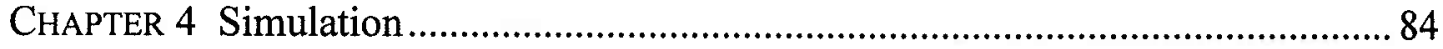

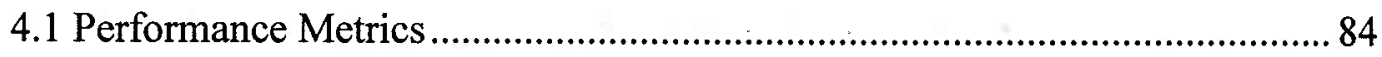

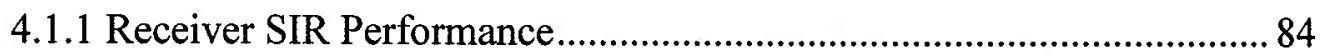

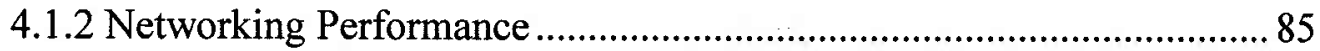

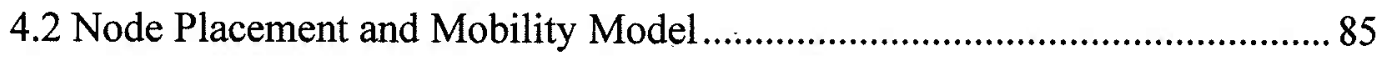

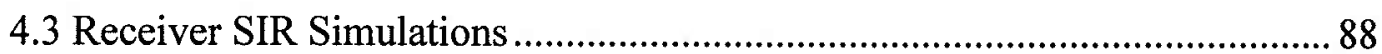

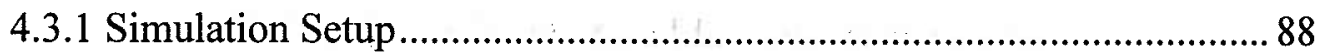

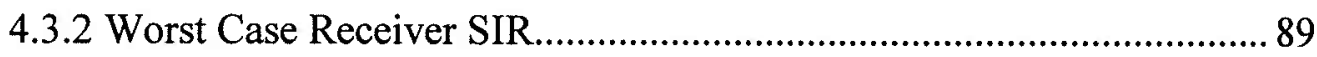

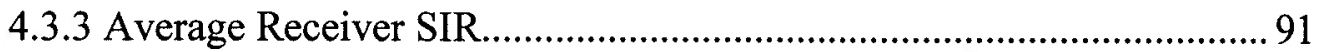

4.4 Networking Performance Simulations ........................................................... 94

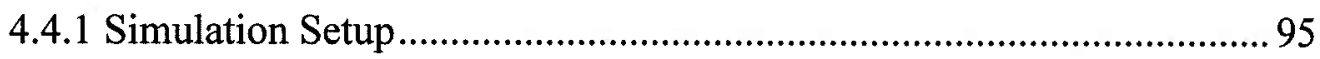

4.4.2 Cell Hopping Parameters and Network Connectivity............................. 96

4.4.3 Node Position Errors and Network Stability ..................................... 100

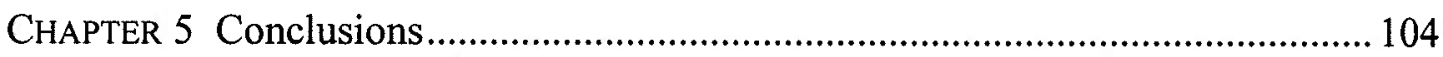

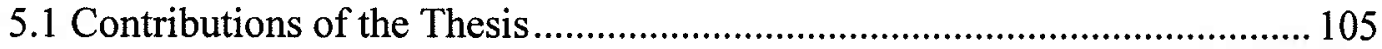

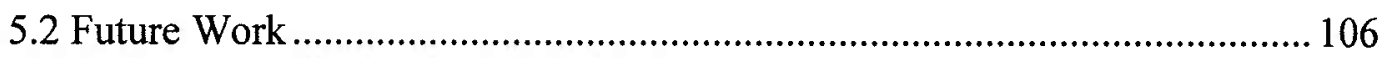

APPENDIX A Spatially Dynamic Cellular Clustering Pseudocode............................ 108

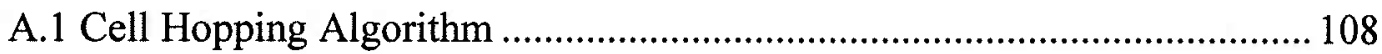

A.2 Cell Membership Algorithm ................................................................ 110

A.3 Node Cell Clustering Algorithm............................................................... 113

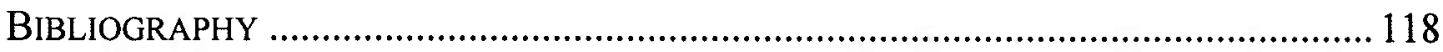




\section{List Of Figures}

Figure 1-1 Military application of underwater acoustic communications technology. The information from land, air, space, and sea assets can be readily correlated by operators in the Tactical Infromation Network center to provide component commanders with an accurate detail of the undersea battlespace picture.

Figure 1-2 Placement of relay nodes in a centralized network. The master node provides an information gateway between nodes in the UAN an operators on the surface or land. The anchored relay nodes coordinate communications between nodes that are beyond the communications coverage area afforded by the master node.

Figure 1-3 Comparison between a centralized and distributed network. The blue arros indicate the links that are actively engaged in communications and the red dotted lines indicate the available links that are not active at this time. In a centralized network only the master node and the slave node can communicate at one time, whereas in a distributed network several pairs of nodes many communicate at any given time.

Figure 1-4 Comparision between the relative coverage areas of a centralized and distributed network. Any node located outside the maximum communications range of either the master or relay nodes is unable to communicate in a centralized network. In a distributed network, several paths exist between nodes provided the range between nodes does not exceed the maximum communications range of the acoustic modem. 
Figure 1-5 Example of a complete hopping cycle using the spatially dynamic cellular clustering scheme. A hopping pattern with 3 clustering points was used in this example. (a) The distributed clustering algorithm clusters nodes into co-located cells using the position information of one-hop neighbor nodes. Once clusters, the nodes within a cell can communicate using the resources allocated to that specific geographic cell. (b) The cellular pattern is repositioned to allow new node clusters to be formed by previously adjacent cells. (c) The process is repeated for each hopping point in the cellular hopping pattern. At the completion of the hopping cycle the process of cell shifting and re-clustering has generated multiple communication paths between all nodes within the network.

Figure 2-1 Seawater absorption versus frequency.

Figure 2-2 Homogeneous fluid layer bounded by arbitrary horizontally stratifieid media with plane wave reflection coefficients $R_{S}$ and $R_{B}$. The proximity of a vertical boundary to the source and the receiver in a shallow water channel gives rise to multiple propagation paths. 39

Figure 2-3 Example of the impulse response and frequency response for an UACC. 38

Figure 2-4 Functional block diagram of a direct sequence spread spectrum (DSSS) transmitter (top) and receiver (bottom).

Figure 2-5 Example of 3 orthogonal PN codes generated using Walsh-Hadamard sequences with a spreading factor of 8

Figure 2-6 Functional bloch diagram of a frequency hopping spread spectrum (FHSS) transmitter

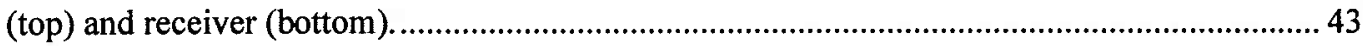

Figure 2-7 Functional block diagram of a RAKE based DSSS receiver.......................................... 46

Figure 2-8 Functional block diagram of a hypothesis feedback equalizer. ........................................ 47

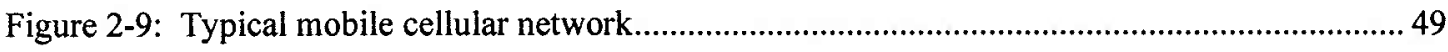

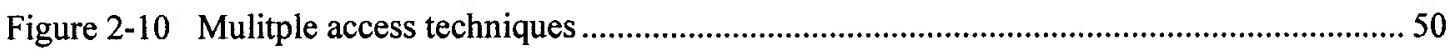

Figure 2-11 Cluster geometry. (a) clusters of sizes of 3, 7, and 12, (b) clusters of reuse size $N=7$ combined to cover a larger geographic area, and (c) the cell coordinate system........................5 52

Figure 2-12 Interfering transmissions between adjacent base stations..............................................5 53 
Figure 3-1 Comparison between a traditional cellular system (a) and a distributed cellular system (b).

The transmitting nodes are represented in red while the receiving nodes are in green. .57

Figure 3-2 Allocation of $\mathrm{PN}$ codes in a $\mathrm{K}=7$ cell reuse pattern. The number assigned to each cell represents the specific PN code that nodes can use to communicate in the co-located geogrpahic area. PN codes are reused as the cell clusters are adjoined to cover a larger geographic area. The cell reuse distance is the minimum distance between cells using the same PN code.

Figure 3-3 Transmitter (red), receiver (green), co-channel interferers (blue), and adjacent channel interferers (black) in a distributed cellular network

Figure 3-4 Demonstration of a full hop cycle and the resultant network routing graph for Node 1.

The solid red arrows indicate the next hop point. The red arcs in the Node Routing Graph indicate available 2 hop paths to distant nodes.

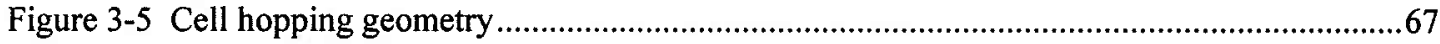

Figure 3-6 Determination of cell membership in a $\mathrm{K}=7$ cell reuse pattern........................................69

Figure 3-7 Node cell membership matrix for 6 node in a $\mathrm{K}=7$ cell reuse pattern...............................70

Figure 3-8 Minimizing the effects of the near-far problem with pre-clustering. (a) The distributed clustering algorithm compares the range proximity between each pair of nodes in the network to determine if a potential near-far problem exists. (b) Those nodes that are within too close of a proximity to one another are pre-clustered together as a supernode (red ring). (c) The clustering algorithm will uses these supernodes to then cluster nodes accoringly. .72

Figure 3-9 Example of a node clustering conflict. The position of th nodes within the orange circle are know to node $\mathrm{A}$

Figure 3-10 TDMA with reservations timing diagram. The maximum propagation delay guard time used in UANs consumes a significant portion of the total slot time .79

Figure 3-11 Diagram of how successive transmission priority scheme can reduce the time wasted from waiting for the channel to clear.

Figure 3-12 Transmission timing diagram for a distributed cellular UAN of 4 nodes using a hybrid MSAP MAC protocol. The red dashed lines indicate the reset point of the minislot timing at each node for a significant transmission event. 
Figure 4-1 Node distributions used in the simulation of the spatially dynamic cellular clustering algorithm. The evenly distributed placement of nodes is useful for simulating a specific node density. The node placement in the random star distribution closely mimics the dispersion of several mobile nodes deployed from a specific point. The random index distribution is a general pupose distribution that is used to define the starting node placements for a random walk simulation. The nearly straight-line appearance of the random sequence distribution is used to simulate the placement of air dropped nodes.

Figure 4-2 Example of a random walk using the parameters $\Theta$ and $\Gamma$ to define the next northing and easting position of a node. 88

Figure 4-3 Worst-case positioning of transmitter (red), receiver (green), co-channel interferer (blue), and adjacent channel interferer (black) in a $K=7$ cell reuse pattern. The figure on the left shows the relative position of the co-channel interferers while the one on the right shows the adjacent channel interfererd. The red ring represents the proximity limit between the receiver and nearby adjacent channel nodes.

Figure 4-4 Worst case SIR versus processing gain for $\mathrm{K}=7,12$, and 19 cell reuse patterns 89

Figure 4-5 A comparison between the average time above SIR for co-channel and adjacent channel interferers. Adjacent channel users are the predominant source of interference at the receiver 91 Figure 4-6 Average time above SIR for $\mathrm{K}=7,12$, and 19 cell reuse patterns. The close spcaing of the SIR curves stems from the fact that the dominant component of the interference at the receiver is generated by adjacent channel users and is independent of the cell reuse pattern .... 92

Figure 4-7 Receiver SIR example for an UAN cosisting of 8 nodes

Figure 4-8 Diagram of hopping points (hop_points) used in simulation. The hopping distance (hop_distance) using the diagram was set to $75 \%$ of the cell radius (cell_radius). The blue cell is centered on the network reference coordinate with each red circle representing the relative shift, or "hop", in the layout of the cell pattern.

Figure 4-9 Diagram of hopping distance (hop_distance) for a 3 hopping point pattern. The blue cell is centered on the network reference coordinate with each red circle representing the relative shift, or "hop", in the layout of the cell pattern. 
Figure 4-10 Network connectivity versus node density for hopping points of $2,3,4$, and 6 . The hopping distance is set to $75 \%$ of the cell radius..

Figure 4-11 Network connectivity versus node density for hopping distances equal to $25 \%, 50 \%$, $75 \%$, and $100 \%$ of the cell radius and a hopping point pattern of 3 .

Figure 4-12 Uncertainty in the position of a node. The red ring represents the area of uncertainty (AOU) generated by node 1 for each of its nearest neigbor nodes. The size of the AOU is based on the maximum speed of the node, the age of the last fix, and the accuracy of the navigation equipment. 100

Figure 4-13 Position error versus average number of link changes for the 4 different node distributions using 36 nodes. The results show that if the maximum error in a node's position does not exceed $21 \%$ of the cell radius then a stable network structure can be maintained by the distributed clustering architecture. 102 


\section{List of Acronyms}

$\begin{array}{ll}\text { ASW } & \text { Anti-Submarine Warfare } \\ \text { AUV } & \text { Autonomous Underwater Vehicle } \\ \text { AOU } & \text { Area of Uncertainty } \\ \text { BPSK } & \text { Binary Phase Shift Keying } \\ \text { CDMA } & \text { Code Division Multiple Access } \\ \text { CML } & \text { Concurrent Mapping and Localization } \\ \text { CTS } & \text { Clear to Send } \\ \text { DADS } & \text { Deployable Autonomous Distributed System } \\ \text { DSP } & \text { Digital Signal Processing } \\ \text { DSSS } & \text { Direct Sequence Spread Spectrum } \\ \text { EM } & \text { Electromagnetic } \\ \text { FBE } & \text { Fleet Battlegroup Experiment } \\ \text { FDMA } & \text { Frequency Division Multiple Access } \\ \text { FEC } & \text { Forward Error Correction } \\ \text { FHSS } & \text { Frequency Hopping Spread Spectrum } \\ \text { FRONT } & \text { Front Resolving Observational Network with Telemetry } \\ \text { FSK } & \text { Frequency Shift Keying } \\ \text { GPS } & \text { Global Positioning System } \\ \text { ISDN } & \text { Integrated Services Digital Network } \\ \text { LAN } & \text { Local Area Network } \\ \text { LBL } & \text { Long Baseline } \\ \text { MAC } & \text { Media Access Control } \\ \text { MAI } & \text { Multiple Access Interference } \\ \text { MFSK } & \text { Multiple Frequency Shift Keying } \\ \text { MSAP } & \text { Minislotted Alternating Priorities } \\ \text { MSC } & \text { Mobile Switching Center } \\ \text { PG } & \text { Processing Gain } \\ & \end{array}$




$\begin{array}{ll}\text { PN } & \text { Pseudonoise } \\ \text { PRD90 } & 90 \% \text { Probability of Detection } \\ \text { PSTN } & \text { Public Switched Digital Network } \\ \text { RTS } & \text { Request to Send } \\ \text { SIR } & \text { Signal to Interference Ratio } \\ \text { SNR } & \text { Signal to Noise Ratio } \\ \text { SLAM } & \text { Simultaneous Localization and Mapping } \\ \text { SSP } & \text { Sound Speed Profile } \\ \text { TDMA } & \text { Time Division Multiple Access } \\ \text { UACC } & \text { Underwater Acoustic Communications Channel } \\ \text { UAN } & \text { Underwater Acoustic Network } \\ \text { UTM } & \text { Universal Transverse Mercator } \\ \text { VLF } & \text { Very Low Frequency }\end{array}$




\section{Chapter 1}

\section{Introduction}

\subsection{Motivation}

The autonomous underwater vehicle (AUV) has evolved to become the preeminent research platform for exploring our undersea world. Archaeological exploration of ancient shipwrecks, detailed hydrographic mapping of deep-sea structures, and mine/counter-mine warfare within denied littoral waters are just a few of the missions that today's AUVs are engineered to perform. Although many AUVs are equipped to execute missions with little or no operator interaction, there still exists a need to efficiently communicate their findings in real-time for data processing and analysis by their human counterpart. At the same time, recent advances in spread spectrum underwater acoustic modem technology have greatly enhanced our ability to implement robust, moderate-capacity digital communication links between nodes operating within a harsh shallow water environment. The advantages of spread spectrum acoustic modems over their conventional fixedfrequency counterparts include excellent interference immunity, multi-user capability, and a lower probability of signal intercept $[2,3]$. The combination of these two emerging technologies has led many military and civilian organizations to 


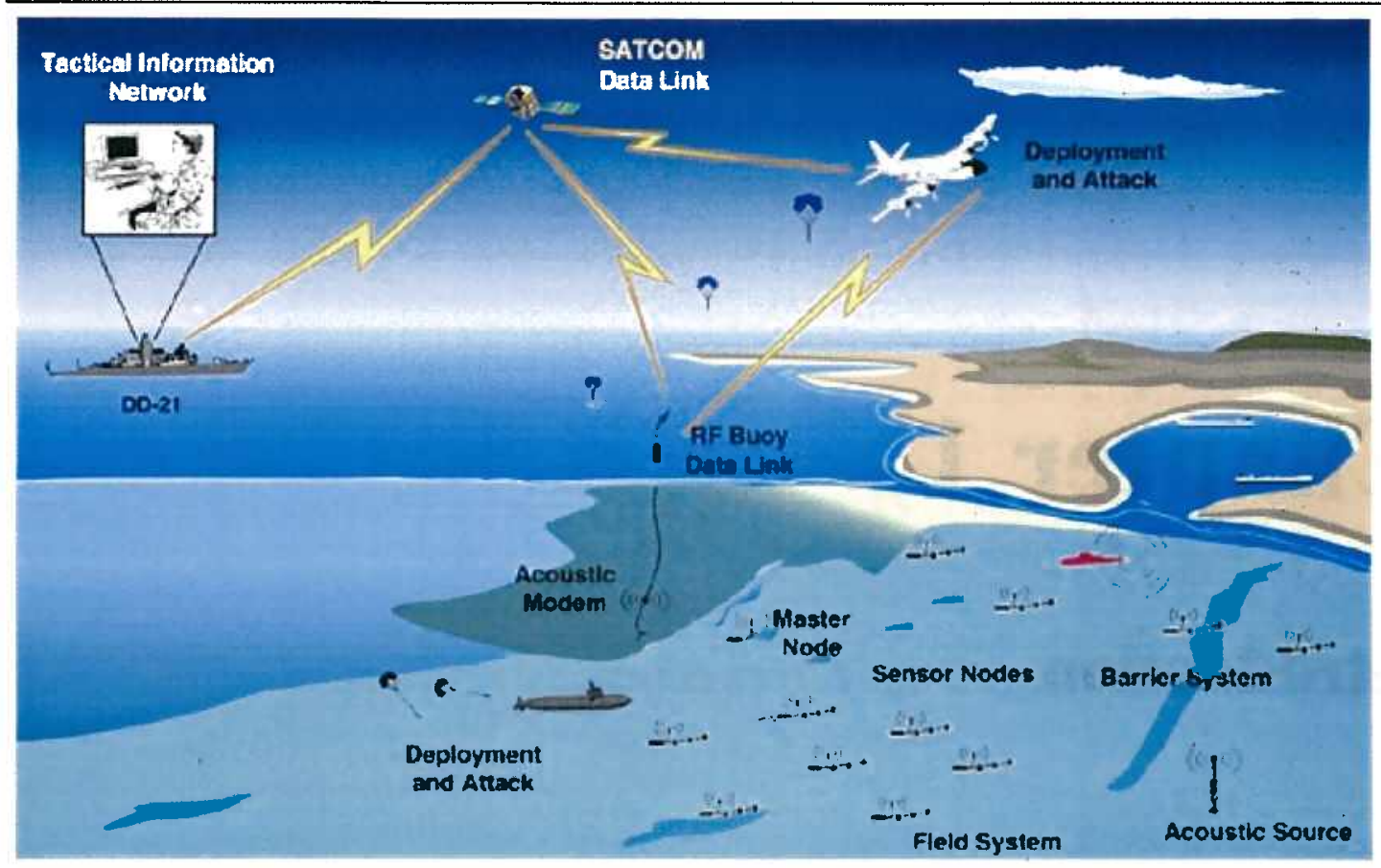

Figure 1-1 Military application of underwater acoustic communications technology. The information from land, air, space, and sea assets can be readily correlated by operators in the Tactical Infromation Network center to provide component commanders with an accurate detail of the undersea battlespace picture.

explore the development of rapidly deployable networks of undersea sensors that can provide sustained in-situ observations from within the harsh ocean environment. An example of how tactical intelligence from land, air, space, and sea assets can be exchanged during the conduct of coordinated anti-submarine warfare (ASW) operations can be seen in Figure 1-1. In the example, the operations of the enemy submarine (red) are closely monitored by the deployed network of undersea sensor nodes and barrier systems. These nodes communicate their finding back to a master node using acoustic modem links. The master node then relays this information onto air and surface assets via satellite using a RF buoy data link coupled to an acoustic modem. The combat commanders can then process the intelligence gathered and transmit the correlated information to ASW assets both above and below the surface for use in prosecuting the enemy submarine. 
Motivation

\subsubsection{Centralized Communication Networks}

A key component of the above example that deserves further examination is the method in which the various undersea sensor systems are networked together using acoustic modems. The underwater acoustic network (UAN) formed by these nodes is a crucial link in the sharing of valuable undersea intelligence. In the example, the UAN employs a centralized network topology whereby the centrallylocated master node coordinates all communication transactions among the network nodes. In order for a sensor node to transmit it must first receive permission from the master node using either a request-to-send (RTS) clear-to-send (CTS) protocol or by being directly polled for data by the master node. The master node is typically located such that it can provide adequate acoustic communications coverage area to

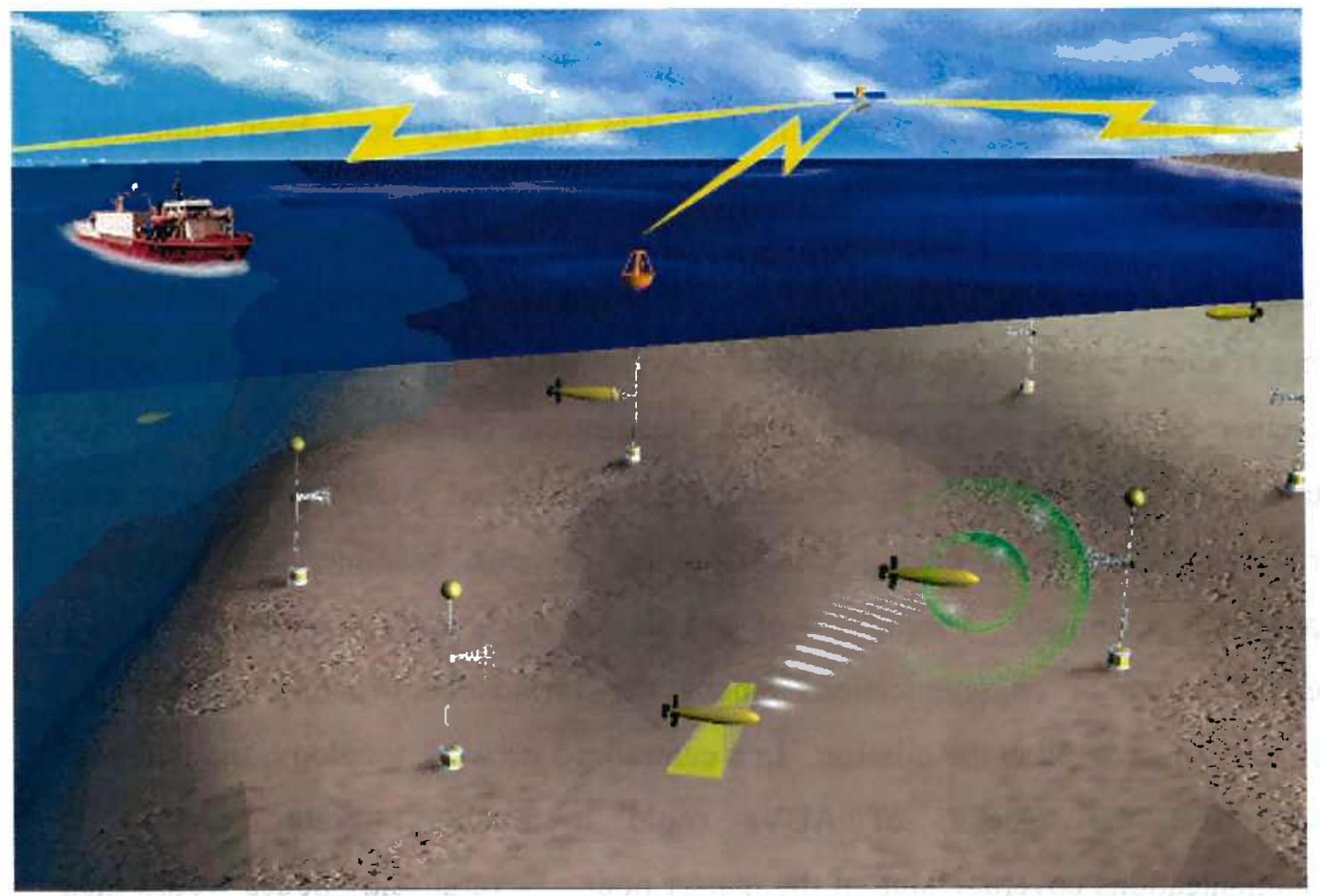

Figure 1-2 Placement of relay nodes in a centralized network. The master node provides an information gateway between nodes in the UAN an operators on the surface or land. The anchored relay nodes coordinate communications between nodes that are beyond the communications coverage area afforded by the master node. 
all deployed nodes in the network. In situations where the coverage area required by the network exceeds the maximum effective acoustic communications range of the master node a series of fixed relay nodes must be deployed, similar to the setup found in Figure 1-2. Although simple in design, a centralized network topology has three major disadvantages:

1. The master node (and relay nodes) can only provide a limited communications coverage area to the deployed network

2. The failure of the master node will result in a complete communications failure of the deployed network (network failure), or in the case of relay nodes, the failure of a relay node will result in a complete communications failure of those nodes it provides coverage (network degradation)

3. Nodes may only transmit their data when they have been authorized (polled) by the master node

Consider the case where multiple AUVs are cooperatively searching for submerged objects along a vast stretch of coastline. Several of the AUVs are tasked to search together in "packs" to provided object localization, detection and classification support for one another. In addition, other specialized AUVs are tasked with providing navigational cueing using moving acoustic baselines and precision navigation equipment. These types of coordinated operations, by virtue, demand a certain degree of communications among the various contributing members to effectively accomplish the mission. In a centralized network topology, the potential exists that the "packs" of AUVs could eventually migrate beyond the communications coverage area of the master node. Several relay nodes would then need to be pre-deployed in conjunction with the AUVs to provide an adequate 
communications coverage area, increasing communications equipment complexity, cost, and deployment and retrieval times.

\subsubsection{Distributed Communication Networks}

In contrast to centralized network topologies, decentralized or distributed adhoc networks are self-organizing, rapidly deployable multi-hop wireless networks that adaptively modify the structure of the network in response to node mobility, node additions, and node removals [4]. Unlike centralized network topologies, communications between nodes in a distributed ad-hoc network require no fixed infrastructure and do not rely upon specialized relay nodes for traffic routing. Instead, each node functions not only as a host, but also as a router that maintains communication routes to and forwards data packets from other nodes in the network that may not be within direct transmission range of one another. A comparison between a centralized network topology and a distributed network topology can be seen in Figure 1-3. In a centralized network topology, only the master node and the slave node can communicate at one time, or the master node may permit the slave

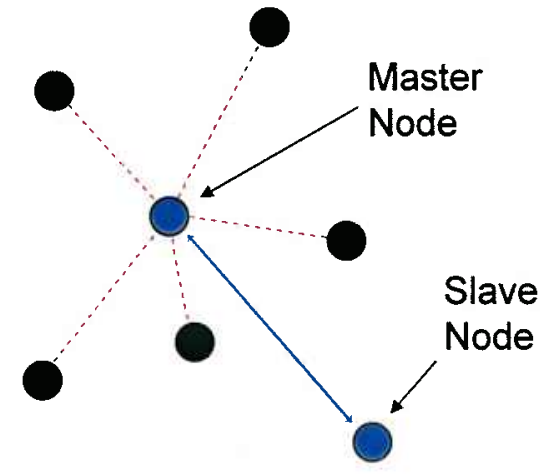

Centralized

Network

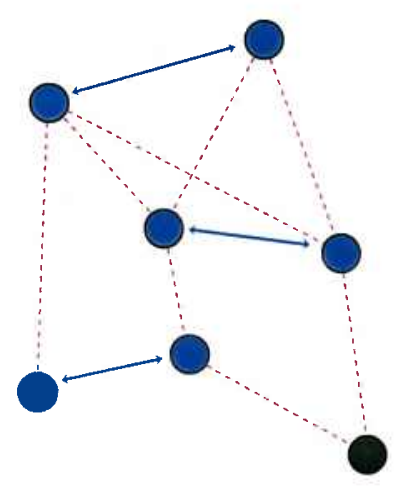

Distributed

Network

Figure 1-3 Comparison between a centralized and distributed network. The blue arros indicate the links that are actively engaged in communications and the red dotted lines indicate the available links that are not active at this time. In a centralized network only the master node and the slave node can communicate at one time, whereas in a distributed network several pairs of nodes many communicate at any given time. 


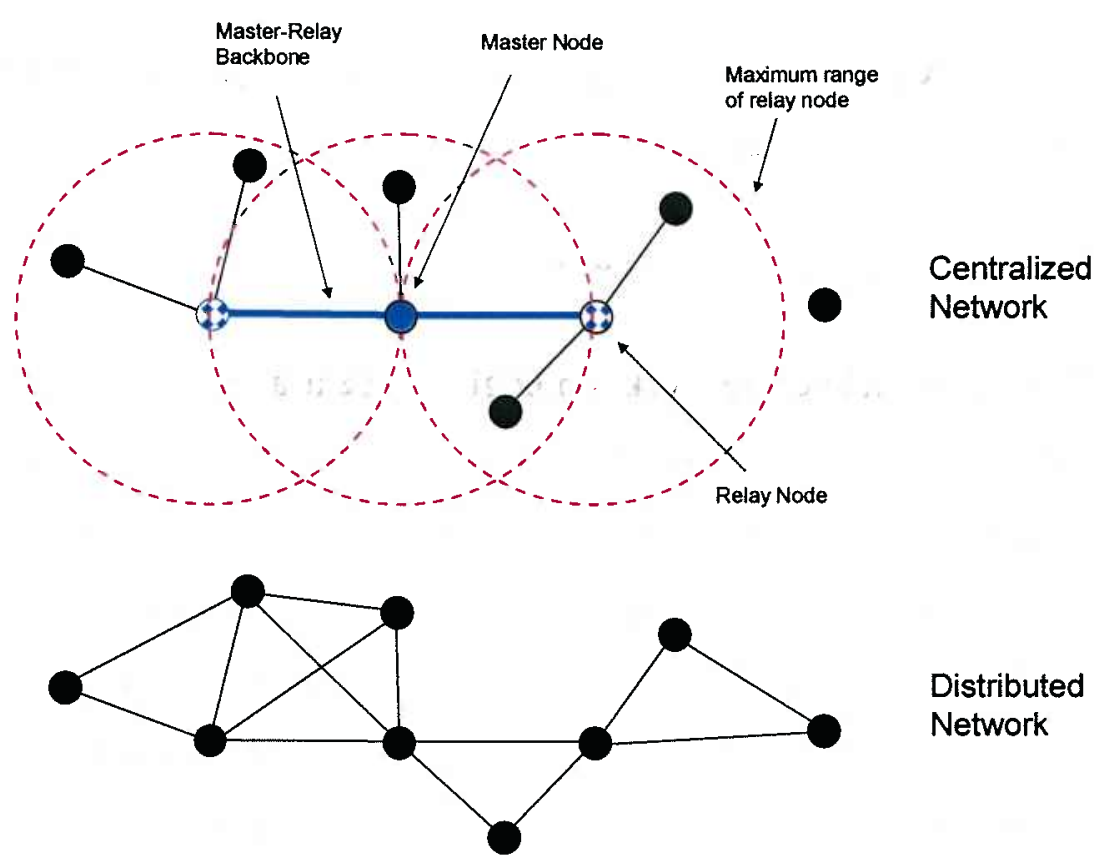

node to communicate with another slave node during that period of time. In the

Figure 1-4 Comparision between the relative coverage areas of a centralized and distributed network. Any node located outside the maximum communications range of either the master or relay nodes is unable to communicate in a centralized network. In a distributed network, several paths exist between nodes provided the range between nodes does not exceed the maximum communications range of the acoustic modem.

distributed network topology several pairs of nodes can be actively engaged in communications at any given time. In general, there are more available paths for communications between nodes in a distributed network than in a centralized network.

Ad-hoc networks also provide a relatively larger communications coverage area since the network's span is determined by the number of member nodes and not by the distance to the nearest master or relay node. Figure 1-4 illustrates a comparison between the relative coverage areas of a centralized and distributed network topology. In a centralized network, those nodes located outside the communications coverage areas serviced by both the master and relay nodes are unable to communicate within the network. All nodes within a distributed network 
Complexities of Underwater Acoustic Network Design

can communicate provided the maximum range between any pair of nodes does not exceed the maximum communications range of the acoustic modem. In addition, the use of shorter multi-hop links between adjacent nodes in the distributed network can reduce the amount of energy consumed in the transmission of packets. The combination of these advantages highlights the attractiveness of the distributed network topology for use in the development of an UAN.

\subsection{Complexities of Underwater Acoustic Network Design}

The underwater acoustic communications channel (UACC) poses several daunting challenges to UAN designers not often encountered in other communication channels. Unlike electromagnetic waves (EM) in air, the available bandwidth and range of acoustic signals within the UACC is highly dependent upon the transmission losses between the source and the receiver, and is generally limited to frequencies below $30 \mathrm{kHz}$ [2]. Within this already limited bandwidth the acoustic signals are subject to reverberation that corrupts the acoustic signal at the receiver, subsequently leading to bit errors in the received transmission. The combination of a relatively low bandwidth and a higher probability for signal errors limits the practical data rate for digital communications in an UAN to around 2-20 kbps with an effective range of only a few kilometers.

In general, the deployment of instrumentation and sensor systems in the ocean and on the seafloor for an extended period of time is primarily limited by the capacity of the on-board battery. It is important that the design and operation of sensor systems strive to be as energy efficient as possible to minimize the power drain on the battery. The UAN protocol must also do its part to be battery efficient by minimizing the number of acoustic modem transmission required for network management. 
In addition to remaining parsimonious of communication, processing, and power resources the networking protocol must also determine network structure for coordinating the transfer of information between nodes. A priori knowledge of network topology is essential for network management and communication resource allocation in a distributed network [5]. The process of topology discovery and routing in ad-hoc networks has been greatly enhanced with the advent of the Global Positioning System (GPS) and other progressive undersea navigation techniques, such as long baseline navigation (LBL) and simultaneous localization and mapping (SLAM). The ability of undersea sensor systems to triangulate and fix their position using these techniques is a significant advantage over wireless radio network designs that lack navigation cueing.

The relatively recent emphasis on synoptic, spatially sampled oceanographic surveillance has provided the impetus to the transfer of networked communication theory to the UACC [2]. Unfortunately, many of the high-efficiency networking protocols employed in modern wireless radio systems cannot be directly applied to the channel. The efficiency of these protocols stems from the fact that the signal propagation delay presented by EM waves in air is negligible ( $5 \mu$ s over $1.5 \mathrm{~km}$ ) when compared to the transmission time of the data packet. Since the time it takes a packet to reach its destination is very small, the networking protocol can allot a dedicated portion of the communications channel for coordinating channel access without incurring a significant reduction in the efficiency of the network. In contrast, the signal propagation delay inherent to acoustic waves in water is significant when compared to the packet transmission time and cannot be ignored. The relatively slow speed of acoustic waves in water produces propagation delays that are five orders of magnitude slower than their EM counterparts in air. Over a distance of $1.5 \mathrm{~km}$ the propagation delay is as much as second! In addition, the relatively low data rates in the UACC produces a packet transmission time that can also be measured in seconds. The networking protocol cannot afford to dedicate a 
Complexities of Underwater Acoustic Network Design

portion of the already limited communication resources to network management without a significant loss in network efficiency. The tradeoff between network management and network efficiency is the fundamental obstacle in the design of a networking protocol suitable for use in the UACC [6].

\subsection{Spatially Dynamic Cellular Clustering}

Spatially Dynamic Cellular Clustering is a novel distributed UAN protocol suitable for ad-hoc deployments of both stationary and mobile nodes dispersed across a relatively wide coverage area in a shallow water environment. The overall network architecture is similar in design to a modern mobile cellular network, except devoid of such fixed infrastructure as the centrally-located transmitter towers and interconnecting control stations.

Each communication node in the network employs the same spread spectrum acoustic modem capable of switching between multiple communications channels using orthogonal signaling techniques. Communication channel reuse throughout the network is accomplished through the geographic separation of cells. All cells within the network are geometrically identical and positioned relative to a common reference coordinate. Nodes communicating within a given cell exercise contentionfree access to the localized communication channel by using a hybrid demand assignment MAC protocol based on Kleinrock's mini-slotted alternating priorities (MSAP) [7] and time division multiple access (TDMA) protocols. 


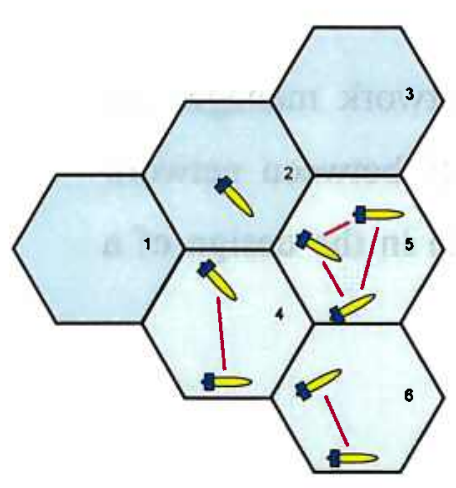

(a)

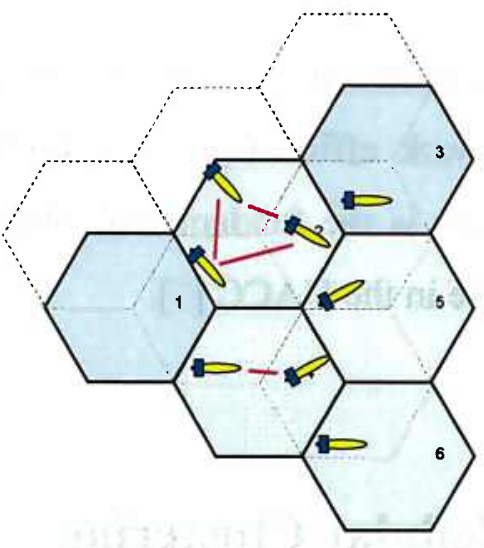

(b)

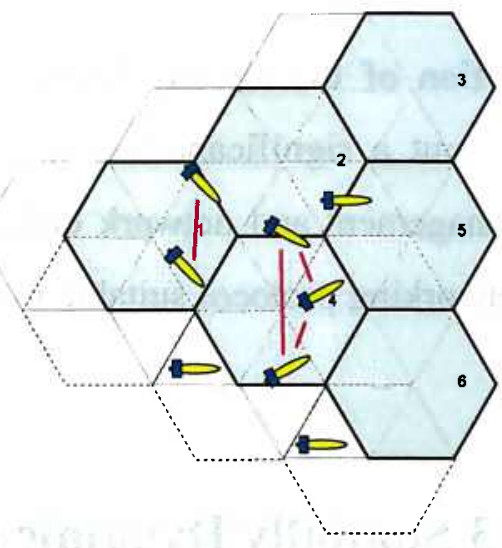

(c)

Figure 1-5 Example of a complete hopping cycle using the spatially dynamic cellular clustering scheme. A hopping pattern with 3 clustering points was used in this example. (a) The distributed clustering algorithm clusters nodes into co-located cells using the position information of one-hop neighbor nodes. Once clusters, the nodes within a cell can communicate using the resources allocated to that specific geographic cell. (b) The cellular pattern is repositioned to allow new node clusters to be formed by previously adjacent cells. (c) The process is repeated for each hopping point in the cellular hopping pattern. At the completion of the hopping cycle the process of cell shifting and re-clustering has generated multiple communication paths between all nodes within the network.

Nodes are dynamically clustered into cells based on their estimated global positions using a distributed clustering algorithm. In order to maintain an up-to-date account of node-to-node link states each node will periodically transmit its global coordinate position to neighboring nodes as either separate packets or imbedded within other types of packets. Each node executing the distributed clustering algorithm requires only a priori knowledge of one-hop neighbor nodes and not the position of every node within the entire network in order to cluster nodes and coordinate network connectivity. Inter-node connectivity among those nodes that are clustered within differing cells is accomplished by periodically relocating, or "hopping", the cell boundaries and then re-clustering nodes. An example of a complete hopping cycle using the spatially dynamic cellular clustering algorithm can be seen in Figure 1-5. Within a bounded period of time, the overall effect of cell boundary hops and node clustering will result in full communications connectivity among all network nodes. 


\section{Complexities of Underwater Acoustic Network Design}

Network parameters, such as cell reuse pattern, cell radius, cell hopping pattern, and minimum and maximum link distance are characterized for general performance, optimized for the given UACC model, and then constrained through simulation to meet the following criterion:

1. Maximize signal-to-interference ratio (SIR) at a user

2. Maximize network connectivity

3. Maximize network stability

The contribution of this thesis is the development and validation of an distributed ad-hoc networking protocol suitable for use in an UAN based upon proven concepts extrapolated from classical cellular communications and network theory and conformed to the modeled UACC. The distributed networking protocol will address the problem of communication resource allocation, communication channel reuse, and the implementation of a MAC protocol suitable for use in a shallow water environment.

Although experimental data is preferred, validation of the network design is performed using simulations only due to the scale, complexity and cost required in physically implementing and testing the network design under in-water conditions. Where applicable, actual experimental data is provided to justify design selections and substantiate conclusions.

\subsection{Related Work}

Although the concepts of cellular design have been widely published, the distributed approach to mobile underwater acoustic networking presented in this thesis is unique. Nevertheless, this thesis does draw from a number of related papers that have been published recently. The work related to this thesis is classified into 
Introduction

three major areas: adaptive clustering for ad-hoc networks, underwater acoustic networking, and spread spectrum signaling within the UACC.

\subsubsection{Adaptive Clustering for Ad-hoc Networks}

Adaptive clustering for ad-hoc networks deals with issues associated with bandwidth allocation, channel reuse, and packet routing. The proposed clustering scheme by Lin and Gerla [8] describes how various code division multiple access (CDMA) techniques, coupled with distributed node clustering, can be used to control access to communication resources within a mobile ad-hoc network. The clustering algorithm uses node ID numbers to deterministically cluster nodes that are reachable via two-hop paths into non-overlapping groups. The paper indirectly addresses the deleterious effects of interference from other user transmissions on network performance by comparing network connectivity versus the transmission range. The interference from other user transmissions is often referred to as multiple access interference (MAI) in the wireless networking community. Their results have shown that as the average transmission range between nodes increases, the connectivity among the nodes increases due to the reduced user interference. While this result is not surprising to cellular designs, it does show that there exists an exploitable link between adaptive distributed clustering and cellular networking.

The clustering scheme presented in [9] details a framework for dynamically organizing mobile nodes in a wireless ad-hoc network into clusters in which the probability of path availability can be bounded. The paper also demonstrates that under moderate levels of mobility the clustering algorithm converges to a stable network topology. More importantly, the mobility-based model used in the simulation of their network protocol provides valuable incite into the temporal and spatial variations in routing paths due to node mobility and density. 
Complexities of Underwater Acoustic Network Design

\subsubsection{Current Underwater Acoustic Network Designs}

Several of the UANs currently in use employ a centralized network topology whereby a centrally-located node coordinates all communication transactions among deployed network nodes. The Deployable Autonomous Distributed System (DADS) is designed to provide undersea surveillance in littoral waters [10]. It consists of an underwater array of fixed sensor platforms interconnected by acoustic modems. Acoustic data is propagated through the network over multi-hop communications paths. The individual hops are configured as half duplex Code Division Multiple Access (CDMA) links between discrete modem pairs. Messages are relayed between paired platforms to minimize the transmit power requirements and reduce the impact of temporal, spatial, and frequency spreading of the signal as it propagates through the UACC. The network connects the remote sensor platforms to a command center through a gateway that relays data received from the acoustic network to the distant command facility across satellite links.

The Front-Resolving Observational Network with Telemetry (FRONT) is a non-military application of DADS that aims to accelerate technology development for numerous critical Navy applications such as littoral ASW and autonomous operations [11-13]. FRONT involves an operating environment, node spacing, and data rates similar to those of DADS and other undersea warfare applications. During the June 2001 Fleet Battle Experiment India (FBE-I), many of the DADS refinements implemented for FRONT were demonstrated in conjunction with two prototype DADS sensors networked with a shore-based ASW command center and a submerged US Navy fast-attack submarine. While qualitative results of the experiment were not readily available, the anecdotal evidence revealed the communications portion of the experiment was the ability to effectively link both undersea and land-based assets into an UAN. 
In general, a centralized network topology provides nodes with fair, contention-free access to the communication channel by allowing only one node to transmit at any given time. While DADS and FRONT are both effective communication network architectures for disseminating data within a centralized network of nodes, its quality of service is limited by high-latency telesonar links that use a request-to-send (RTS), clear-to-send (CTS) MAC protocol. Consequently, network performance in terms of data latency and throughput efficiency is diminished using the prescribed MAC protocol as nodes must wait in turn to transmit.

A series of papers by Sozer et al [3, 14] outline the development and simulation of a centralized network protocol for an ad-hoc network of static nodes in a shallow water environment using OPNET Modeler/Radio. Their work detailed the considerations required in the discovery and initialization phases of an UAN protocol regarding topology discovery and node routing algorithms. These papers also address the key relationship between the operation of the network protocol and battery life, and provides qualitative simulation results comparing network activity with battery consumption.

A fixed assignment distributed network protocol described by Stojanovic et al [15] addresses the exploitable relationship between network management and vehicle navigation. The protocol uses Time Division Multiple Access (TDMA) to allow multiple AUVs to locate one another by measuring inter-vehicle signal propagation delays and by exchanging self-generated localization maps. The propagation delay information gathered among the AUVs can then be used in concurrent mapping and localization (CML) operations as well as path discovery for routing data packets within the network. The paper also investigated a soft limit on 
the number of AUVs that can be effectively employed by this protocol per square kilometer and still be able to meet the desired communication timing criteria ${ }^{1}$. The limit calculation accounted for the maximum speed of the AUVs, the desired localization map update rate, and the maximum expected distance between AUVs. While this particular protocol is well suited for a small group of AUVs tasked to conduct a moving group search, the use of TDMA as the multiple access method complicates the scalability of the UAN as there are no provisions for large-scale channel reuse. In addition, the temporal and spatial variations of the UACC can significantly affect the accuracy of inter-vehicle signal propagation delays. In this case, the use of on-board navigation functions is more ideally suited to triangulate the position of vehicles in the network.

\subsubsection{Spread-Spectrum Acoustic Modems}

Until recently, acoustic telemetry has relied primarily on incoherent modulation techniques such as frequency shift keying (FSK) to provide robust digital communications in an undersea environment. While FSK is effective in low data rate applications, the need for higher data rates has shifted acoustic mode design towards more spectrally efficient techniques found in coherent modulation. An excellent overview on the evolution of acoustic telemetry from incoherent to coherent modulation methods can be found in [2].

Smaller, more efficient digital signal processors (DSP) has facilitated the migration of spread-spectrum signaling from the airwaves into the UACC. There are many relevant papers addressing the design, implementation, and testing of spread-

\footnotetext{
${ }^{1}$ key timing criteria include: localization map update rate, TDMA slot length, and maximum channel guard time
} 
spectrum acoustic modems [1, 16-20]. In general, many of the same design principles used in a common cellular phones can be found in these modems, making their integration into UAN designs less rigorous.

\subsection{Thesis Organization}

The thesis is divided into five chapters, including this introduction. Chapter 2 provides the relevant background material used in the development of this thesis. It discusses the fundamental characteristics of the UACC as a communications medium, spread-spectrum communication techniques, and the cellular concept. Chapter 3 details the technical approach taken in this thesis in the design of an adhoc UAN using spatially dynamic cellular clustering. Chapter 4 discusses the network and UACC simulation models, collected data, and network performance analysis metrics. Finally, chapter 5 summarizes the simulation results, presents conclusions drawn from this work, and discusses future extensions. 


\section{Chapter 2}

\section{Background}

This chapter provides the requisite background knowledge considered necessary in the development of the spatially dynamic cellular clustering scheme presented in this thesis. The physical limitations of the UACC as communications medium are discussed, and then related to its fundamental application in acoustic telemetry design. Spread-spectrum communication theory is presented in terms of parameters that directly affect multiple user communications in a harsh communications channel similar to the UACC. Finally, an overview of classical mobile cellular theory is presented with an emphasis on communication channel reuse.

\subsection{Underwater Acoustic Communications}

The physical characteristics of water as a communications medium complicate the design of an UAN. While mediums such air or space allow electromagnetic (EM) waves to propagate relatively unimpeded over vast distances throughout nearly the entire radio spectrum, seawater severely attenuates these waves through absorption processes to effective ranges of not more than a few meters [21]. Although radio signals below the very low frequency (VLF) band can 
be received at great distances underwater, the high-power antennae required are very large and the effective data rates are extraordinarily low making EM waves an impractical carrier for networked digital communications within the UACC. For these reasons, acoustic waves are predominantly used for digital communications in the UACC.

The UACC is typically characterized as a random time-varying medium that is bandwidth-limited and reverberant to acoustic waves [2]. The available bandwidth and range of sound within the UACC is highly dependent upon transmission losses from both attenuation and geometric spreading, and is generally limited to frequencies below $30 \mathrm{kHz}$. Within this limited bandwidth, the acoustic signals are subject to time-varying multipath propagation and Doppler spreading that corrupts the acoustic signal by inducing inter-symbol interference (ISI) and signal fading at the receiver. In addition, the relatively slow speed of sound in water produces propagation delays that are five orders of magnitude slower than that of their EM counterparts in air. Accordingly, the combination of these physical characteristics complicates digital communication network design in the UACC.

\subsubsection{Attenuation Losses}

The attenuation of acoustic energy is a combination of both absorption from the various molecular loss mechanisms associated with seawater and scattering interactions from marine biologics, entrained air bubbles, and vertical channel boundaries. The frequency-selective absorption of acoustic energy in seawater is the dominant component of transmission loss at the ranges and frequencies of typical acoustic modems and stems from three principle loss mechanisms: shear viscosity, structural relaxation of water, and ionic relaxation of salts [21]. The frequencydependent nature of these loss mechanisms can be characterized using the semiempirical expression for seawater absorption derived by Thorp [22] 
Underwater Acoustic Communications

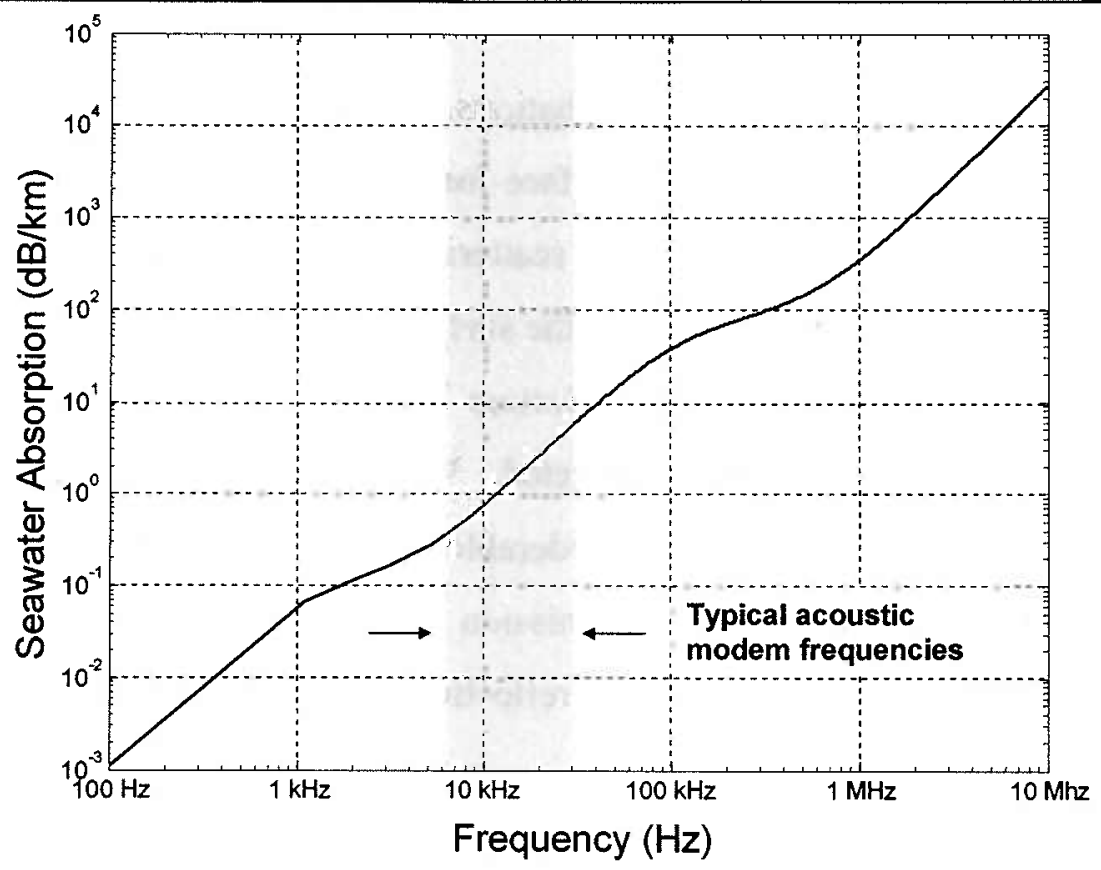

Figure 2-1 Seawater absorption versus frequency.

$$
\alpha=1.094\left(\frac{0.1 f^{2}}{1+f^{2}}+\frac{40 f^{2}}{4100+f^{2}}+2.75 \times 10^{-4} f^{2}+0.003\right)(\mathrm{dB} / \mathrm{km})
$$

where $\alpha$ represents the coefficient of absorption loss and $f$ is the center frequency of the acoustic signal in kilohertz. A plot of seawater absorption versus frequency can be seen in Figure 2-1. The transmission loss $(H)$ from absorption between a source and a receiver separated by a distance $r$ can then be calculated by

$$
H=\alpha r
$$

As attenuation through absorption is the more limiting factor in transmission loss, a practical range limit in acoustic telemetry designs can be found using the empirical criterion [21]

$$
\alpha r=10 d B
$$

The interaction of acoustic waves with their channel boundaries can introduce significant scattering losses. The scattering losses incurred due to interactions with the ocean surface are exceedingly variable due to their dependence 
on the magnitude and period of wave perturbations. In calm seas, acoustic waves are perfectly reflected by the free-release surface formed at the high-impedance airsea boundary. However in rough seas, the scattering losses increase dramatically due to the variability in the specular angle at the surface, particular at the small-scale levels associated with higher frequencies. Bottom loss is a measure of the energy lost into bottom as the acoustic wave is reflected. While bottom loss in deep-water applications is not crucial, it can have a considerable effect on transmission losses in shallow water applications [23]. The transmission loss due to bottom interactions (bottom loss) is a function of the plane wave reflection coefficient $\left(R_{B}\right)$ for the given bottom type and is defined as

$$
H=20 \log \left|R_{B}\right|
$$

In this thesis, the effects of surface scattering and bottom loss will be neglected by assuming these losses are deterministic when compared to the dominant effect of spatial variability from absorption and spreading losses associated with mobile nodes within a uniform UACC. In addition, the range limit of the acoustic modems employed within the network design is based upon the $90 \%$ probability of detection limit for the given UACC conditions, which effective masks the losses from scattering.

\subsubsection{Geometric Spreading Losses}

The degree of transmission loss due to geometric spreading depends upon the ratio of the range between the source and receiver (r) and the ocean depth (D). In close range, deep water applications $(\mathrm{r} / \mathrm{D}<1)$ the geometric spreading losses are due primarily from spherical spreading with the source and the receiver connected by a direct-path eigenray. The transmission loss from spherical spreading can be calculated by

$$
H=20 \log r
$$


In shallow-water littoral applications $(\mathrm{r} / \mathrm{D}>1)$ spherical spreading gives way to cylindrical spreading as acoustic wave propagation is constrained to within the vertical boundaries of the channel. The transmission loss between the source and the receiver is now defined as

$$
H=10 \log r+10 \log \left(D \cos \theta_{i}\right)
$$

where $\theta_{\mathrm{i}}$ is the minimum incident angle between the empirically dominant eigenray and the vertical boundary. The first term represents the losses over the entire range due to cylindrical spreading while the second term represents the additional loss incurred due to spherical spreading prior to the wave front interacting with the vertical boundary.

As an acoustic signal propagates from the source to its intended receiver, its energy is degraded by the combination of both frequency and range dependent transmission losses in seawater. In order for the receiver to properly detect and decode the transmission the signal strength of the received signal must be sufficiently stronger than the noise presented by other interfering sources in the UACC. The ratio between the signal power received and the noise power from other sources is known as the signal to noise ratio (SNR). Lower SNR levels result in a higher probability that the receiver will be unable to successfully decode the transmission without errors. If either the range between the source and receiver or the frequency of the acoustic signal increases, then the amount of transmission loss increases and the available SNR at the receiver will decrease. The Shannon-Hartley theorem states that the capacity $(C)$ of a communications channel, in bits per second, is a function of the average received SNR and the bandwidth $(W)$, and can be stated as

$$
C=W \log _{2}(1+S N R)
$$


It is theoretically possible to transmit information over the UACC at any bit rate $(R)$, where $R \leq C$, and incur a small bit error probability by using a sufficiently complicated coding scheme and a higher transmission power [24]. For these reasons the practical bandwidth of the UACC is generally limited to frequencies below 30 $\mathrm{kHz}$.

\subsubsection{Sound Speed}

Spatial and temporal variations in the temperature, pressure, and salinity of seawater result in widely dynamic sound speed profiles (SSP) that are uniquely characterized in trait, time, and location. In deep water, variations in SSP are dominated by temperature in the upper 1000 meters and hydrostatic pressure in the deep ocean. In the upper tens of meters, the SSP is heavily influenced by solar heating and wind, and can vary on a daily basis [23]. Directly below this layer is the thermocline, followed by an isothermal region that extends to the ocean floor. Acoustic waves generated within the region below the thermal layer can become trapped, propagating vast distances without ever reflecting from a vertical boundary. Comparatively, acoustic waves generated in the region above the thermal layer alternately reflect from the surface towards the bottom only to be bent upward.

In shallow water less than $100 \mathrm{~m}$ a seasonal thermal layer is generated in the upper 5 meters followed by a nearly isovelocity profile that extends to the seafloor. Depending on the geographic location and sufficient mixing from winds and currents the SSP may be completely isothermal. The UAN protocol developed in this thesis has been tailored to operate within the shallow water acoustic channel $(<100 \mathrm{~m})$ where the communication nodes are located within a nearly isovelocity SSP. 


\subsubsection{Reverberation}

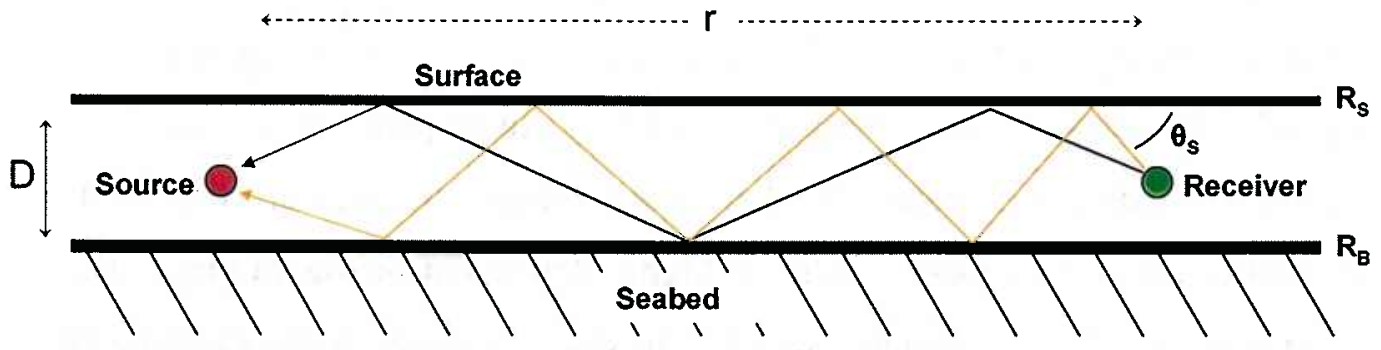

Figure 2-2 Homogeneous fluid layer bounded by arbitrary horizontally stratifieid media with plane wave reflection coefficients $R_{S}$ and $R_{B}$. The proximity of a vertical boundary to the source and the receiver in a shallow water channel gives rise to multiple propagation paths.

Reverberation is a result of acoustic signals being spread in both time and frequency by the existence of multiple propagation paths and relative motion between the source and the receiver. The presence of a free-release surface, a hard bottom, and a depth-dependent sound speed profile (SSP) gives rise to multipath propagation, especially in shallow water channels $(\mathrm{r} / \mathrm{D}>1)$. Figure $2-2$ provides an illustration of the multiple propagation paths present between a source and a receiver in a shallow water channel. Differences in reflected path lengths cause signal transmissions to arrive at the receiver with a finite temporal scattering called the delay spread $\left(T_{d}\right)$ of the channel. The channel delay spread is typically specified as the interval during which $S(\tau)$ remains essentially nonzero $[24,25]$. Intuitively, delay spread measures the approximate duration of channel "echoes" resulting from multipath arrivals. Figure 2-3 shows the magnitude impulse response of a simulated UACC, along with its corresponding magnitude frequency response. The delay spread of an UACC is
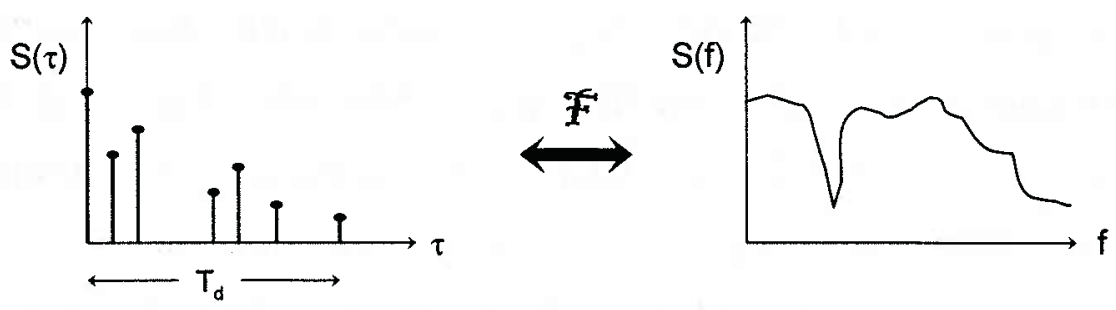

Figure 2-3 Example of the impulse response and frequency response for an UACC. 
typically on the order of $100 \mathrm{~ms}$ or more. A time dispersive UACC generates two potentially deleterious effects at the receiver: frequency-selective amplitude and phase variations (fading) and inter-symbol interference (ISI). Frequency-selective fading is caused by delayed signal components adding out of phase at the receiver. This condition is evident in Figure 2-3 where deep spectral notches are observed. Since the spectral location of these notches is highly dependent on signal phase, the overall channel response is particularly sensitive to changes in receiver location and orientation. Meanwhile, ISI occurs whenever delayed arrivals from one symbol interval "spill over" into subsequent symbol intervals. ISI impedes the ability of the receiver to distinguish the desired signal from the echoes of the previously transmitted symbols.

The inverse of the channel delay spread is called the coherence bandwidth $\left(\mathrm{B}_{\mathrm{coh}}\right)$ of the channel. When the coherence bandwidth is large relative to the signal bandwidth the multipath arrivals are primarily confined to a single symbol interval [25]. In the frequency domain, this corresponds to a channel impulse response that remains approximately constant across the signal bandwidth of interest. This situation is referred to as frequency non-selective, or flat multipath fading. In this case, ISI is negligible and channel equalization can be accomplished using a single complex-valued gain multiplication. In narrowband applications, the primary concern of the receiver is not ISI but the impact of frequency-dependent channel amplitude variations. Since the entire narrowband signal can randomly drop into a deep channel notch, high performance receivers for narrowband signals require fast automatic gain control (AGC) circuitry, large dynamic range, and diversity reception. In wideband applications where the signal bandwidth is larger than the coherence bandwidth of the channel (e.g. spread spectrum) the receiver's primary concern becomes ISI and not the narrowband notches. In this case, the channel is characterized as being a frequency-selective fading channel since the channel magnitude response varies across the signal bandwidth. Signal distortion from 
frequency-dependent fading normally results in severe ISI at the receiver requiring complex wideband equalizers for acceptable receiver performance [26]. When the level of ISI is particularly severe the only solution may be to decrease the maximum symbol rate of a transmission. A general conclusion is that multipath effects increase the required complexity and power consumption of acoustic modems.

The interval over which the impulse response of the UACC remains correlated is called the channel coherence time $\left(T_{\text {coh }}\right)$. The channel coherence time provides a measure of the variation rate in the UACC impulse response. Channels are characterized as being either fast fading or slow fading depending on the length of the coherence time relative to a single symbol interval. The channel coherence times are strongly dependent on the relative motion between the transmitter, receiver, and other objects or surfaces in the channel. In narrowband applications, the fading rate directly affects the responsiveness and dynamic range of the receiver. In wideband applications, the fading rate primarily impacts the required convergence rate for adaptive receiver algorithms [26].

The product of $\mathrm{B}_{\mathrm{COH}} \mathrm{T}_{\mathrm{COH}}$, called the Doppler spreading factor, is used to determine whether the channel is either underspread $\left(\mathrm{B}_{\mathrm{COH}} \mathrm{T}_{\mathrm{COH}}<1\right)$ or overspread $\left(\mathrm{B}_{\mathrm{COH}} \mathrm{T}_{\mathrm{COH}}>1\right)$. In general, the impulse response of a significantly underspread channel $\left(\mathrm{B}_{\mathrm{COH}} \mathrm{T}_{\mathrm{COH}}<<1\right)$ can be readily obtained and exploited to optimize the timevarying performance of the receiver [16]. However, the ability to characterize the rapid fluctuations in the impulse response of an overspread channel is an intractable problem for phase-coherent modulation. 


\subsection{Spread Spectrum Communications}

The following discussion seeks to offers insight into the advantages and application of spread spectrum communications in the UACC. A more in-depth presentation of spread spectrum theory can be found in [24, 27], with [16] providing a more detailed approach to the UACC. Spread spectrum communication techniques are highly attractive for use in an UAN due to their ability to accommodate multiple users at the same time, use of low probability of interception signals, and their relative immunity from the deleterious effects of multipath and Doppler spreading.

The modulation techniques used in spread spectrum communications can be classified into two categories: direct-sequence spread spectrum and frequencyhopping spread spectrum. In a direct-sequence spread spectrum (DSSS) system, the spectral bandwidth of a data sequence of rate $R$ is spread by convolving it with a pseudo-noise (PN) code sequence whose symbol rate is much higher than that of the
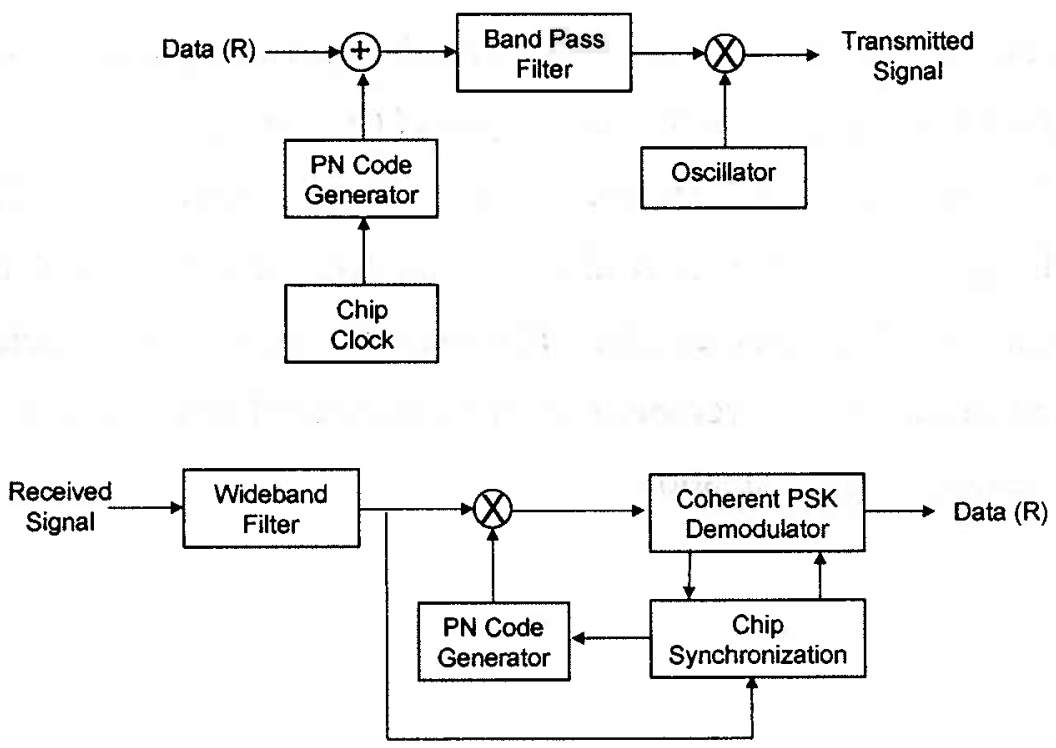

Figure 2-4 Functional block diagram of a direct sequence spread spectrum (DSSS) transmitter (top) and receiver (bottom). 


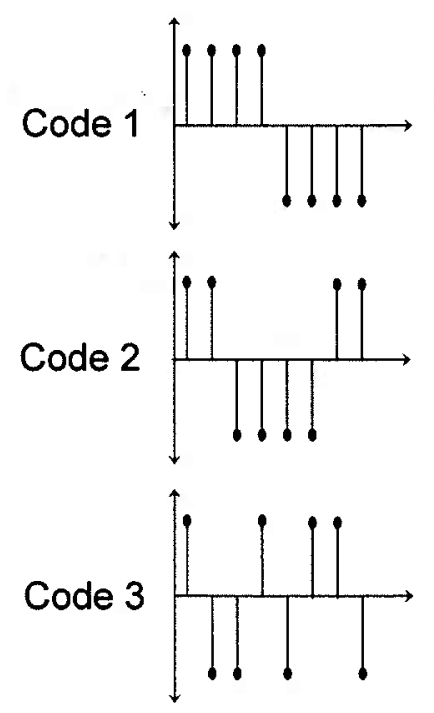

Figure 2-5 Example of 3 orthogonal PN codes generated using WalshHadamard sequences with a spreading factor of 8

data sequence. The resultant wideband pseudorandom signal of bandwidth $\left(W_{S S}\right)$ is then modulated using binary phase shift keying (BPSK) to produce a signal that has noise-like properties when compared to the data sequence. A function block diagram of a DSSS system can be seen in Figure 2-4. Although the resultant signal appears as random noise, each intended receiver can despread the data signal in a deterministic manner through cross correlation when provided with the proper PN code. The length of the PN code sequence is called the spreading factor with individual code elements referred to as chips. Three code sequences selected from the Walsh-Hadamard set are illustrated in Figure 2-5 for a spreading factor of 8. In most spread spectrum systems the code sequence elements are restricted to take on the value \pm 1 to simplify hardware implementation. Following modulation the information bandwidth of the signal is characterized by a factor approximately equal to the spreading factor. This explains the use of the term spread spectrum to describe these systems.

A frequency hopping spread spectrum (FHSS) system spreads the data signal directly in frequency by using multiple frequency shift keying (MFSK) modulation 

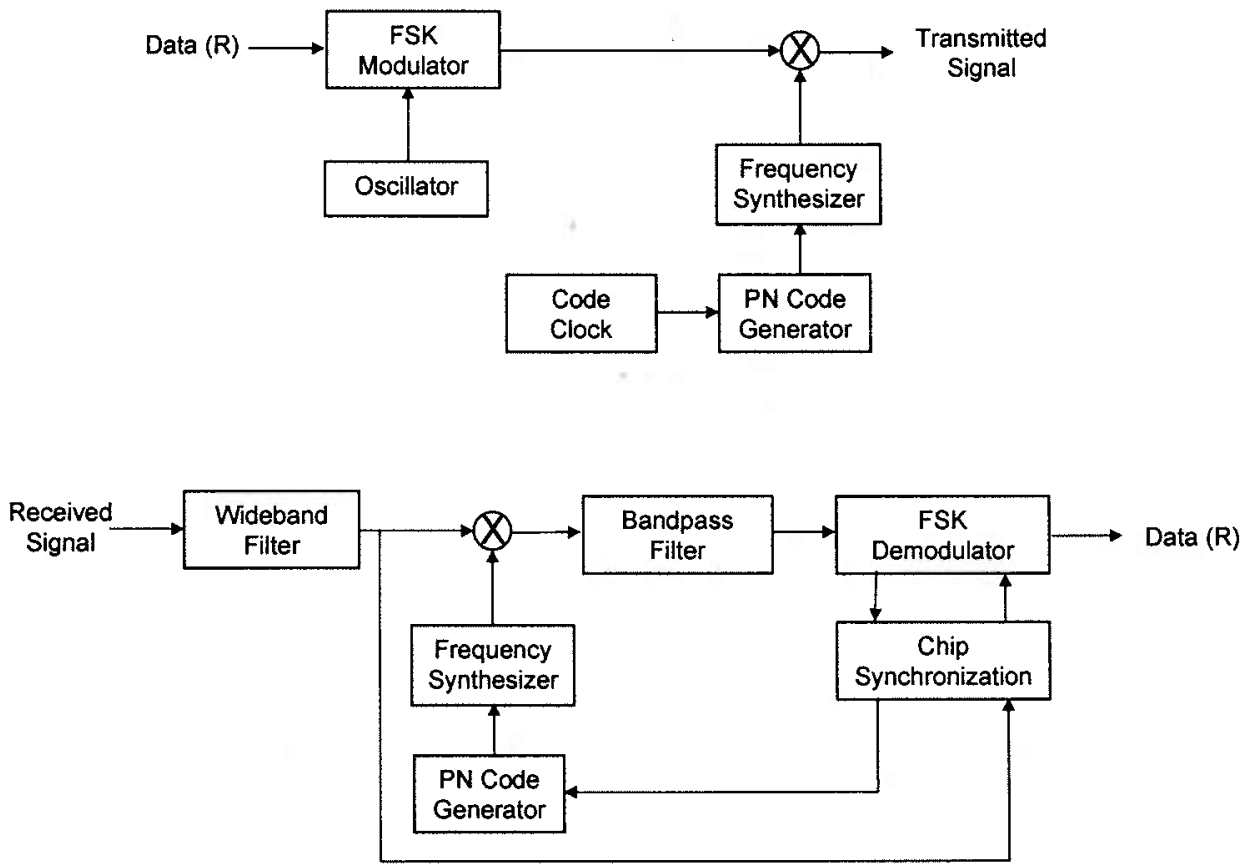

Figure 2-6 Functional bloch diagram of a frequency hopping spread spectrum (FHSS) transmitter (top) and receiver (bottom)

in conjunction with orthogonal frequency hopping patterns. A functional block diagram of a FHSS transmitter and receiver can be seen in Figure 2-6. The transmitter and receiver "hop" from one frequency channel to the next in a predetermined pattern, with each hop lasting for a fixed time duration called the dwell time. FHSS systems are generally characterized as either slow hopping or fast hopping depending on the length of the dwell time. Slow hops typically occur on the order of hundreds or thousands of symbol times while fast hops can be as brief as a few symbol times. In practice, the hopping interval is chosen to be considerably shorter than the coherence time of the channel, reducing the likelihood of channel variations during a single hop. FHSS systems were originally designed for use in secure military communication applications. Since signal detection requires knowledge of the hopping pattern at both transmitter and receiver, information access can be easily restricted. In the presence of fixed-frequency interference or 
jamming an intelligent FHSS system could be engineered to "learn" the channel and interference conditions and then adjust the hopping patterns to avoid these "bad" channels. Despite these attractions, FHSS systems impose considerable complexity on the overall system design. These receivers require an accurate, frequency-agile oscillator capable of pseudo-randomly tuning across the entire system bandwidth. Considerable bandwidth is wasted on every hop because of the overhead required for resynchronization. Finally, coordinated hopping is essential in high performance systems to prevent co-channel interference, but requires tight timing synchronization between users.

While cross correlation of the spread spectrum signal with the correct PN code reproduced the transmitted data signal, the cross correlation of a signal from another user or narrowband interferer appears as a much smaller wideband noise signal at the receiver. Since each user is assigned a unique PN code that is nearly orthogonal to the codes of other users, a receiver can effectively separate each user from within the wide spectral bandwidth by their respective PN codes. Thus, multiple users can simultaneously share the same bandwidth without producing significant multiple access interference (MAI) between one another. Another name more commonly used to describe this multiple access technique is code division multiple access (CDMA). The above result deserves emphasis as it implies that the total number of users in a spread spectrum system is a soft limit based on the maximum tolerable MAI of the receiver, or processing gain. The processing gain $(P G)$ is synonymous with the spreading factor and is given by

$$
P G=\frac{W_{S S}}{R}
$$

The theoretical upper limit on the number of users in a spread spectrum system can be readily derived by comparing the total interference within the system to the signal 
of a given user, or signal-to-interference ratio (SIR). Define the signal power (S) from each user as

$$
S=R E_{b}
$$

where $E_{b}$ is the average energy contained in each transmitted bit. Similarly, assuming the only interference is from other users, let the interference power $(I)$ be defined as

$$
I=W_{\mathrm{SS}} N_{o}
$$

Thus, the generalized expression for the SIR is [24]

$$
S I R=\frac{S}{I}=\frac{R E_{b}}{W_{S S} N_{o}}=\frac{\left(\frac{E_{b}}{N_{o}}\right)}{\left(\frac{W_{S S}}{R}\right)}
$$

If each user controls its transmitter power such that the power of each user at the intended receiver is equal, then the total system interference is

$$
I_{S}=S(M-1)
$$

Substituting $I_{S}$ for I in (2.11), the SIR now becomes

$$
S I R=\frac{S}{S(M-1)}=\frac{1}{(M-1)}
$$

Substituting SIR from (2.13) into (2.13), the capacity of the spread spectrum system is found to be

$$
M \approx(M-1)=\left(\frac{W_{S S}}{R}\right) \frac{1}{\left(\frac{E_{b}}{N_{o}}\right)}=\frac{P G}{\left(\frac{E_{b}}{N_{o}}\right)}
$$




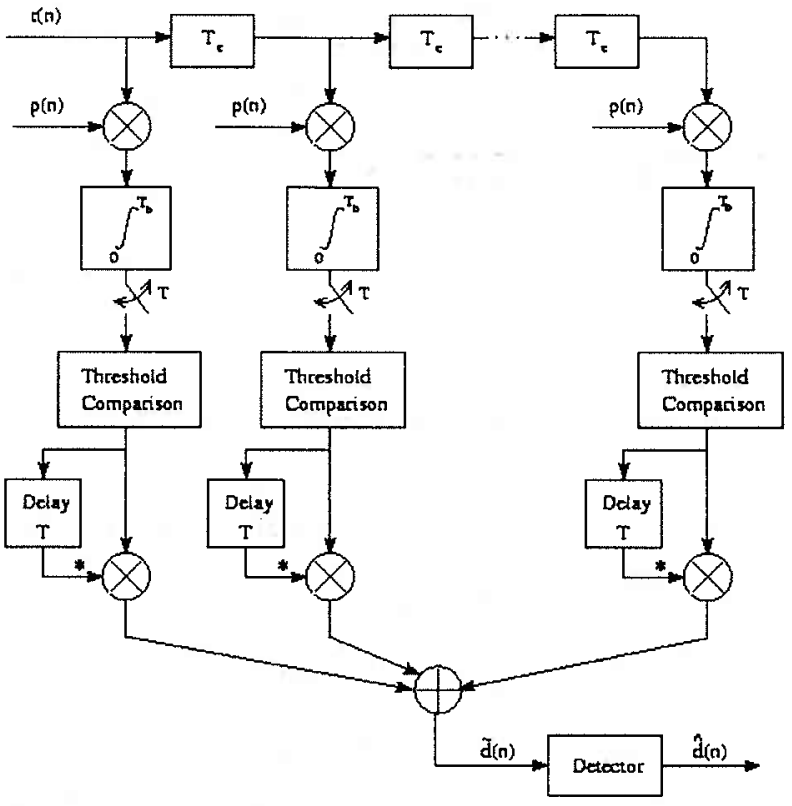

Figure 2-7 Functional block diagram of a RAKE based DSSS receiver [1]

which shows that the theoretic capacity of the system is directly proportional to the to the processing gain, and the SIR in (2.13) for each user is inversely proportional to the total number of simultaneous transmitters. The typical range of acceptable values for SIR in spread spectrum acoustic modems is between $-5 \mathrm{~dB}$ and $5 \mathrm{~dB}[1$, 14].

Multipath propagation and Doppler spreading in the UACC results in a frequency-selective channel due to multiple versions of a transmitted signal arriving at a receiver delayed in time, attenuated in magnitude, and shifted in frequency and phase. Since the energy of a spread spectrum signal is uniformly distributed over the entire bandwidth, only a small portion of the transmitted signal will be lost due to frequency-selective fading. In this case, forward error correction (FEC), interleaving, and other coding techniques are used to recover from the relatively small number of bit errors encountered in the recovered data signal. 


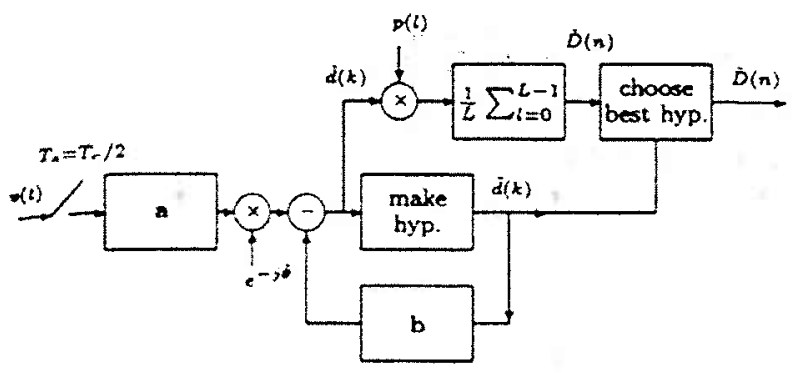

Figure 2-8 Functional block diagram of a hypothesis feedback equalizer [1]

Depending on the fade rate of the communications channel, spread spectrum systems can improve its overall performance by processing the various multipath components using adaptive equalization techniques such as RAKE or hypothesis feedback filtering [1]. The RAKE filter shown in Figure 2-7 consists of a delay line that is tapped over equally spaced intervals by a bank of correlators based on the impulse response of the communications channel. Each correlator produces an output that is shifted in time and weighted in magnitude depending on the particular multipath component it is designated to resolve. The outputs of these correlators are then summed to produce a final estimate of the original signal. A hypothesis feedback adaptive filter estimates the original signal by hypothesizing the value of the data symbol based on the lower mean squared error at the end of each bit interval [1]. Simultaneously, the receiver parameters corresponding to the hypothesized value are retained for decision feedback and synchronization in the next bit interval, thereby making it possible to track and decode signals in a rapidly time-varying channel. A functional block diagram of a hypothesis feedback equalizer can be seen in Figure 2-8. 


\subsection{The Cellular Concept}

The cellular concept for wireless networks grew from the need to accommodate a large number of mobile telephone subscribers sharing limited communication resources. The principle features of the cellular concept are (1) the reuse of the communications spectra in more than one geographic coverage area or cell through the careful control of transmitter power and (2) provision for capacity expansion through the division of cells into smaller cells [28].

A typical mobile cellular network uses a centralized network topology as shown in Figure 2-9. As illustrated, a geographic area can be spanned by using overlapping circular cells. It is common in cellular network planning to use interlocking hexagonal cells vice overlapping circles as they are conceptually easier to visualize and more convenient to manipulate. Centrally located within each cell is a fixed tower or base station that coordinates the communication needs of each mobile user within its cell coverage area. The base stations are interconnected
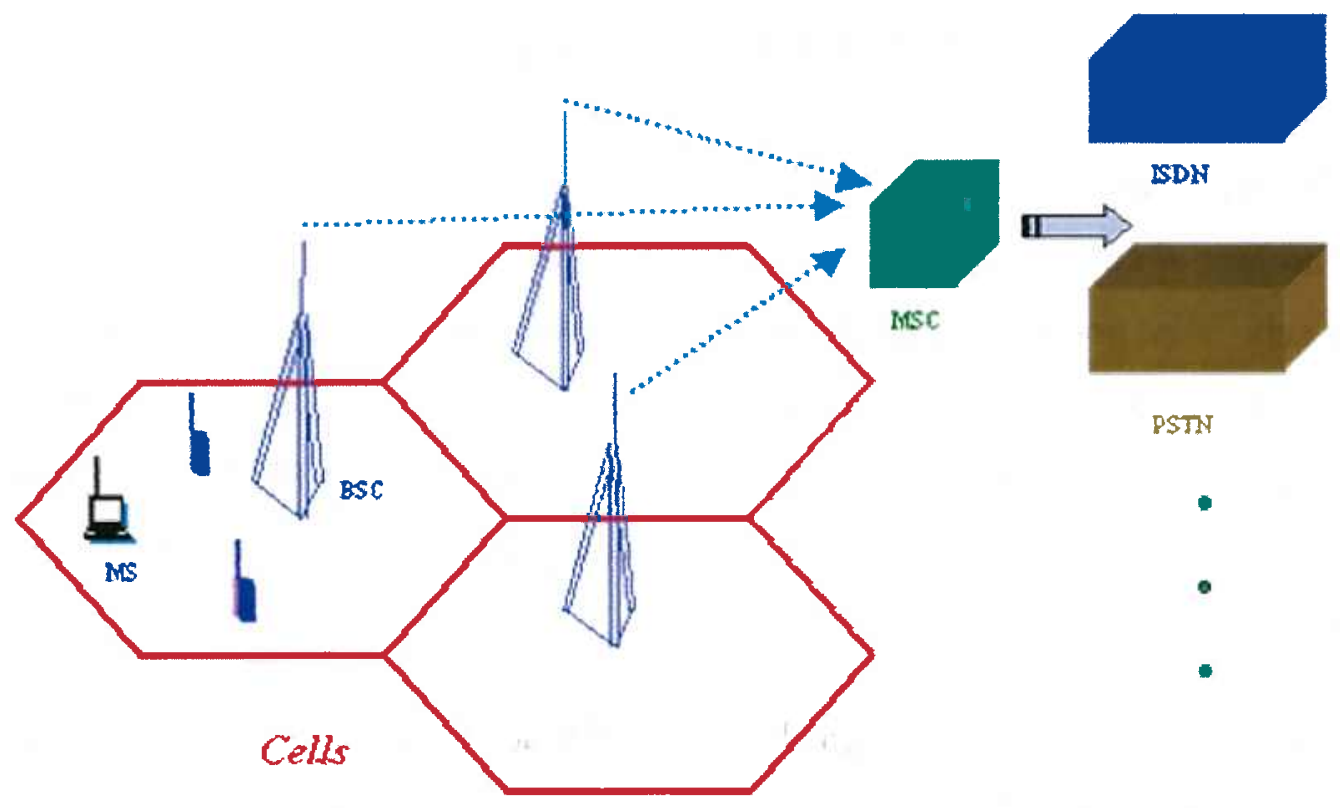

Figure 2-9: Typical mobile cellular network 
through a wired information backbone to a mobile switching center (MSC) that coordinates intracellular communications. In order for a mobile user located in one cell to communicate with another user in a differing cell the call must be routed though their respective base stations and then through the MSC. Calls destined to a landline phone or mobile networks beyond the control of the regional MSC are routed though the local wired public switched telephone network (PSTN) or integrated services digital network (ISDN).

\subsubsection{Multiple Access Techniques in Cellular Systems}

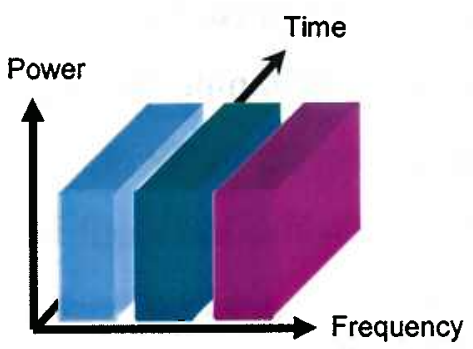

FDMA

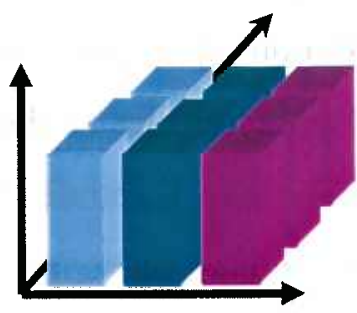

TDMA

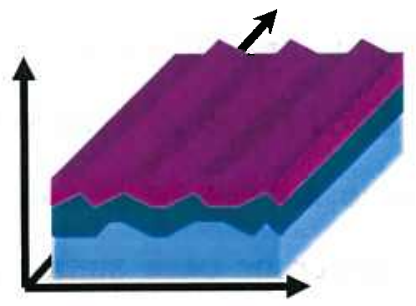

CDMA

Figure 2-10 Mulitple access techniques

Multiple access techniques are used in cellular systems to allow several mobile users to simultaneously share the finite amount of available radio spectrum in a given cell. First generation cellular systems utilized Frequency Division Multiple Access (FDMA) to share the communications channel, while second and newer third generation cellular systems employ Time Division Multiple Access (TDMA) or code division multiple access (CDMA). Figure 2-10 is a graphical representation of each technique in terms of power, frequency, and time.

In FDMA, each user is assigned a unique frequency band with two channels, a forward and a reverse. Base stations use forward channels to carry traffic along to the mobile users, while the mobile users use reverse channels to transport opposite 
traffic. Such a transmission procedure is called Frequency Division Duplexing (FDD). Base stations and mobile stations must use frequency-selective filtering and guard bands in order to distinguish between the intended signal and the interference from adjacent channels. FDMA is suitable for "streaming" traffic because a dedicated portion of the frequency spectrum is always allocated to the user, but becomes inefficient in "bursty" traffic. Designers of the next generation of cellular systems acknowledged that FDMA is too spectrally inefficient to accommodate the ever increasing number of mobile users, since unused channels simply remained idle. Therefore, all second generation cellular systems used either TDMA or CDMA techniques.

TDMA divides the spectrum in time slots so that each user can use as many slots as needed in a cyclical manner to either transmit or receive traffic. Control of bit rates can be achieved by allowing a station to accommodate several slots or by allowing slots to have variable duration sizes. The advantage of this scheme is that users operate over the same frequency, but in different time slots according to availability, so that no part of the spectrum ever remains idle. Each station transmits its traffic during its assigned time slot over a given frequency band. In order to reduce the likelihood of other user interference a guard time is inserted between each user's time slots. Furthermore, duplexers are not required since different time slots are used for transmitting and receiving.

CDMA is used predominant in second generation, and exclusively in third generation cellular systems. As previously discussed, the key feature of CDMA is that all users transmit over the same spectral bandwidth using unique spreading codes being assigned to each user (spread spectrum). The importance of this technique lies in the ability to reuse the entire spectral bandwidth within all cells. In fact, CDMA is the only multiple access scheme that does not require any frequency planning (frequency channel reuse) [28]. 


\subsubsection{Frequency Reuse}

As previously discussed, the cellular concept permits the reuse of the communications spectra in more than one geographic coverage area through the careful control of transmitter power and cell subdivision. In terms of user capacity, we use multiple access techniques to share the limited spectral bandwidth in one geographic area, and then reuse that same bandwidth in a more distant geographic area where sufficient propagation losses between the two areas prevents mutual interference. Thus, frequency planning in cellular networks is primarily a function of characterizing propagation losses and signal-to-interference ratios between stations using the same frequency spectra in different geographic areas.

In frequency planning, each geographic area is represented as a cluster of cells. The number of cells within a cluster, or cluster size, is dependent upon the division and distribution of the frequency spectra. Figure 2-11a illustrates how various cluster sizes based on frequency reuse patterns of $\mathrm{N}=3,7$ and 12 are

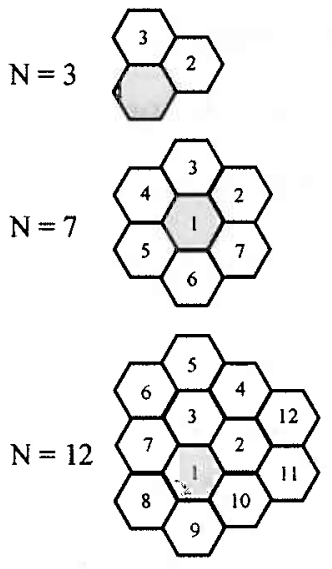

(a)

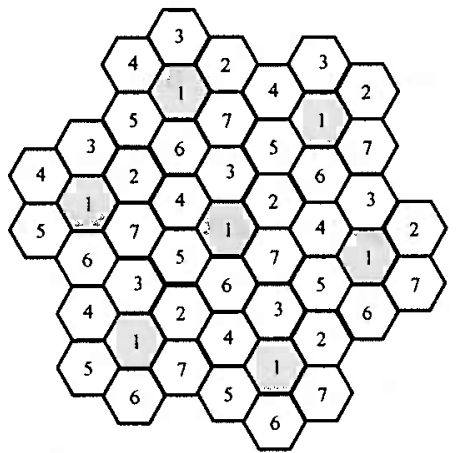

(b)

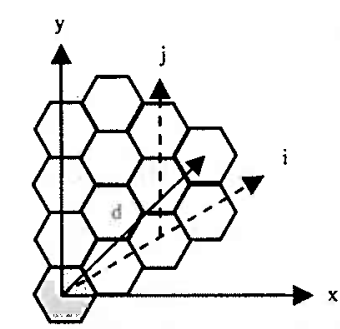

$d(i, j)=\sqrt{i^{2}+i j+j^{2}}$

Figure 2-11 Cluster geometry. (a) clusters of sizes of 3, 7, and 12, (b) clusters of reuse size $\mathrm{N}=7$ combined to cover a larger geographic area, and (c) the cell coordinate system. 


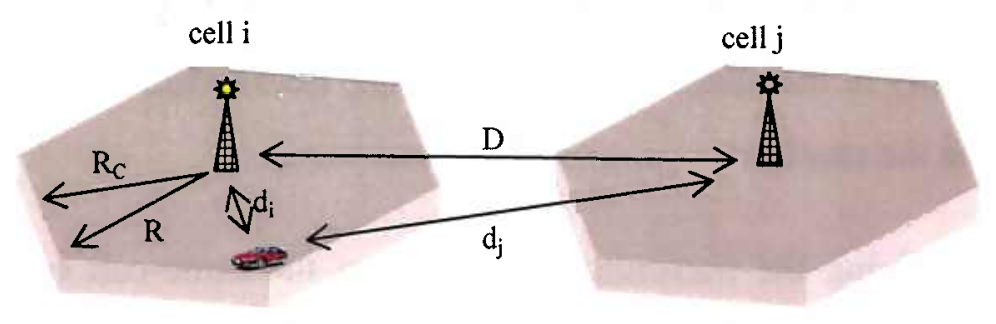

Figure 2-12 Interfering transmissions between adjacent base stations

constructed. The cell number represents the fractional portion of the frequency spectra used within the cell. The geometry of each cluster takes on a compact form and is based upon a cell arrangement such that there is (1) complete coverage of the area within the cluster, and (2) complete coverage of the area once multiple clusters are combined. An example of how multiple $\mathrm{N}=7$ cluster arrangements can be combined to cover a large geographic area is show in Figure 2-11b.

Radio transmissions propagating from the base station tower to the mobile user are attenuated through geometric spreading and frequency-selective fading by the environment. Other random phenomena used to model small-scale variations in the received signal power, such as lognormal shadowing and Rayleigh fading, are minor and will be neglected as they provide little additional insight into the development of this discussion.

In order for the mobile user to detect and decode the transmission, the base station transmitter power must be sufficient to overcome the propagation losses experienced between the tower and the mobile user. Using Figure 2-12, the power received at the mobile user $\left(P_{M}\right)$ from cell $i$ is

$$
P_{M}=P_{T} d_{i}^{-\gamma}
$$

where $P_{T}$ is the base station transmitter power, $d_{i}$ is the distance between the mobile user and the base station tower in cell $i$, and $\gamma$ is the path loss exponent. Typical 
values for the path loss exponent range between 2 and 4 depending on the aggregate composition and density of the materials (e.g., buildings, trees) within the direct path of the transmitter and the receiver.

In addition to receiving the transmitted signal from the same-cell base station tower, the mobile user may also receive interfering signals within the same frequency spectra from adjacent towers located within distant cells, called cochannel interference. The shaded cells in Figure 2-11 represent the co-channel interfering cells in an $\mathrm{N}=7$ cluster reuse pattern. Assuming each base station tower transmits at the same power level, the total co-channel interference power $\left(I_{C O}\right)$ at the mobile user in cell $i$ is

$$
I_{C O, i}=\sum_{j=1}^{6} P_{T} d_{j}^{-\gamma}
$$

As previously discussed, the mobile user is capable of detecting and decoding a transmission provided the SIR is above that required by the receiver. Thus, the SIR for this arrangement is

$$
S I R_{i}=\frac{P_{M}}{I_{C O, i}}=\frac{P_{T} d_{i}^{-\gamma}}{\sum_{j=1}^{6} P_{T} d_{j}^{-\gamma}}=\left[\sum_{j=1}^{6}\left(\frac{d_{i}}{d_{j}}\right)^{\gamma}\right]^{-1}
$$

which shows that the SIR for any mobile user within the cellular network is strictly a function of distance ${ }^{2}$.

\footnotetext{
${ }^{2}$ The result is based on the assumption that each user does not exceed the maximum design transmitter power of the network
} 
In the cell coordinate system, the distance between two cells is given by the equation in Figure 2-11c. Since hexagonal geometry of radius $\mathrm{R}$ is used in frequency planning, the distance between two cells with identical frequency spectra is

$$
d(i, j)=2 R \sqrt{i^{2}+i j+j^{2}}=R_{C} \sqrt{3} \sqrt{i^{2}+i j+j^{2}}=R_{C} \sqrt{3 K}=D
$$

where $K$ is the cell reuse ratio, and $D$ is the cell reuse distance. Interestingly, the cell reuse ratio is identical to the number of cells within a cluster ${ }^{3}$. Substituting (2.18) as an approximation for $d_{j}$ in (2.17) reveals a simplified form for SIR in terms of the reuse ratio.

$$
\operatorname{SIR}=\left[\sum_{j=1}^{6}\left(\frac{R_{C}}{R_{C} \sqrt{3 K}}\right)^{\gamma}\right]^{-1}=\frac{(3 K)^{\frac{\gamma}{2}}}{6}=\frac{\left(\frac{D}{R_{C}}\right)^{\gamma}}{6}
$$

Using a path loss exponent in air of 4.0 in a $K=7$ cell reuse pattern, the SIR for a mobile user within the cellular network would be approximately $18 \mathrm{~dB}$.

Although CDMA based systems do not require frequency planning, they may require code reuse plans that reuse the pseudo-noise (PN) codes in a similar fashion as the approach presented above. In general, PN code reuse plans can be readily reconfigured to accommodate more users by communications software at the base station and mobile user, whereas to reconfigure the frequency limits of the radio equipment in a FDMA or TDMA system would be impractical.

\footnotetext{
${ }^{3}$ This relationship holds true provided the cell cluster arrangement is constructed using an appropriate minimum distance algorithm such as Dikstra's algorithm
} 


\section{Chapter 3}

\section{Spatially Dynamic Cellular Clustering}

\subsection{Network Organization}

The application scenario that motivates this work is the formation of an UAN between nodes that are dispersed in an ad-hoc fashion across a relatively large geographic area in a shallow water environment. The node set consists of both stationary platforms (sensor arrays, information gateway) and mobile platforms (AUVs) that employ the same spread spectrum acoustic modems for network communications. Since the UACC accommodates only limited communication resources, the major challenge in UAN design is to then partition these resources such that each node is afforded fair access to the channel. In cellular phone networks, these resources are monitored and managed by the centrally located control infrastructure in each cell. Nodes within a given cell are made aware of one another's communications capabilities using the same infrastructure. The same cellular concept can be abstracted to ad-hoc networks by creating similar cellular clusters of nodes, but instead allowing communications resources to be controlled in a distributive manner, devoid of the need for a central controlling station. Figure 3-1 provides a pictorial comparison between the tradition cellular network and the one used in a distributed cellular network. In the traditional cellular system, the mobile 


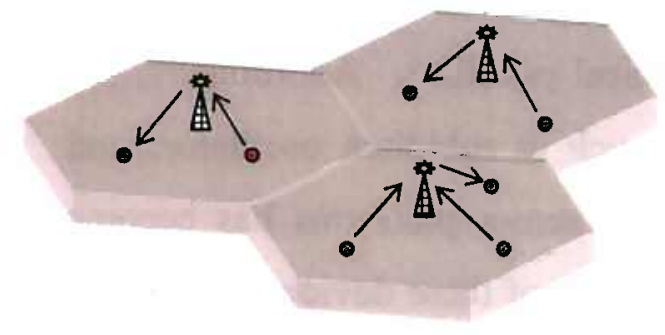

(a)

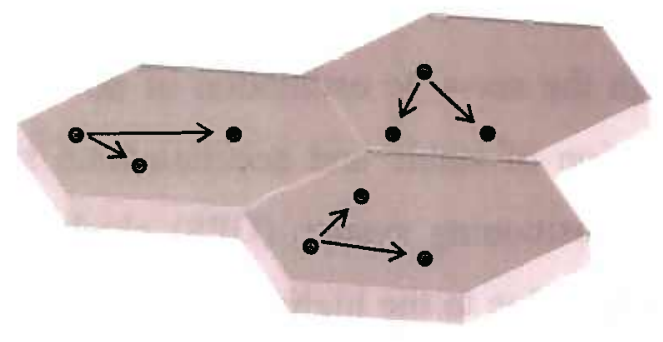

(b)

Figure 3-1 Comparison between a traditional cellular system (a) and a distributed cellular system (b). The transmitting nodes are represented in red while the receiving nodes are in green.

users and the tower in a cell can both transmit and receive at the same time (duplex), whereas in the distributed case, using half-duplex acoustic modems, only one node can transmit at a time in a given cell.

Assuming the geographic operating area of the nodes is subdivided and patterned using geometrically identical cells, the global position of a node can be easily mapped to the co-located area covered by a cell. Using this co-location information, the overhead cost in coordinating resources can then be minimized by allocating specific communication resources for use in each cell. If nodes are provided with the positional information of its nearest neighbors, then they can also be mapped into cells, and an ad-hoc network using distributed clustering can be readily formed.

\subsubsection{Determination of Network Structure}

Unlike cellular phones, the nodes within an UAN will have knowledge of their geographic position using on-board navigation functions making clustering (and communication resource allocation) a deterministic problem. Advances in undersea

navigation, such as long baseline (LBL) ranging and simultaneous localization and mapping (SLAM), coupled with smaller, more efficient form-factors for computational processing can allow for rapid determination of network structure 
through the accurate estimation of node geographical positions. Additionally, the integration of stable and accurate time sources, such as rubidium oscillators and global positioning system (GPS) clocks, into many sensor platforms has become ubiquitous due to the higher availability and lower cost of these devices. Thus, the network design presented here assumes that all nodes are capable of:

1. Fixing their own coordinate position (latitude/longitude/depth) to within a few meters

2. Maintaining clock synchronization with some degree of accuracy to within a few milliseconds

Once each node has fixed its position within the UACC, it can transmit this information to other nodes within reception range. Since knowledge of network structure is crucial to providing shared access to the channel, each node will maintain a table of positions of its nearest neighbors known as the NODEPOSIT table. An example of the relevant data stored in the NODEPOSIT table can be seen in Table 3-1. Nodes will periodically transmit their updated positions and listen for the same from others in an effort to maintain the most up-to-date record of its nearest neighbors. Based on this information, each node can deterministically group nearest neighbor nodes into cells using a distributed clustering algorithm.

Table 3-1 NODEPOSIT Table

\begin{tabular}{|c|c|c|c|c|}
\hline Node Number & $\begin{array}{c}\text { Time } \\
\text { (UTC) }\end{array}$ & Latitude & Longitude & $\begin{array}{c}\text { Depth } \\
\text { (meters) }\end{array}$ \\
\hline 1 & 1023745751.762 & N41.527622 & W70.673423 & 13.2 \\
\hline 3 & 1023745724.521 & N41.523713 & W70.672113 & 43.2 \\
\hline 6 & 1023745776.324 & N41.522212 & W70.671972 & 23.1 \\
\hline
\end{tabular}

While latitude and longitude provide the precise global coordinate of a node, these qualities can become cumbersome to manipulate over the relatively small scale distances of an UAN. A more desirable approach is to convert the position of a node 
Network Organization

from the global coordinate system (latitude, longitude) to the Universal Transverse Mercator (UTM) coordinate system (northing, easting). Using UTM, the northing and easting values can be normalized to a fixed reference point in the UAN without a loss in precision. As such, all coordinate calculations presented in his thesis are in terms of the UTM northing and easting relative to a fixed reference coordinate in the UAN.

\subsubsection{Cell Coverage Area Considerations}

As previously discussed in section 2.3, CDMA cellular systems do not require frequency planning due to the inherent interference immunity found in spread spectrum signaling techniques. The lack of a centralized control infrastructure in the above distributed extension of cellular networking shifts the burden of resource allocation to each and every node in the network. If every node uses the same PN code to communicate, then the network's efficiency degrades to that of a centralized network topology. Alternatively, if one or more nodes wish to communicate, then they must first coordinate which PN code to use. Depending on the method of coordination, this process can result in a significant amount of communication resources being wasted performing network management. Assuming each node is able to fix its geographic position in time, it should then be able to triangulate its position relative to any fixed point in the UACC. Using this notion, and the fact that geometrically identical cells are used in the formation of the distributed cellular network, each node can then triangulate the position of every cell within in the network. Thus, a distributed clustering algorithm can then deterministically group nodes by their positions into cells where the PN code for nodes in that particular geographic region is the same.

The geometric size of the cells used in the cellular network is based on the $90 \%$ probability of detection range limit (PDR90) of the acoustic modem. The 
PDR90 is defined as the range at which the modem has a $90 \%$ probability of detecting and receiving an acoustic transmission without bit errors for a given transmitter power under the given acoustic environmental conditions (e.g., ambient noise, SSP, bottom type, etc.) Consequently, the actual cell size (PDR90) can vary day-to-day with changes in ocean environment or surface weather conditions. In situations where a low probability of signal interception is required, the transmit power of the acoustic modem can be attenuated, thereby reducing the size of the cell.

The cluster size (cell reuse pattern) of the distributed cellular network is based on the number of available PN codes and the minimum SIR required by the modem. Each of these factors directly affects the minimum cell reuse distance. An example how $\mathrm{PN}$ codes are allocated to cells in a $\mathrm{K}=7$ cell reuse pattern can be seen in Figure 3-2. The more PN codes that are available for use the larger the cell reuse pattern, the longer the code reuse distance, and the smaller the interference from those users that are transmitting with the same PN code. Conversely, the lower the minimum SIR required the shorter the cell reuse distance, the smaller the cell reuse

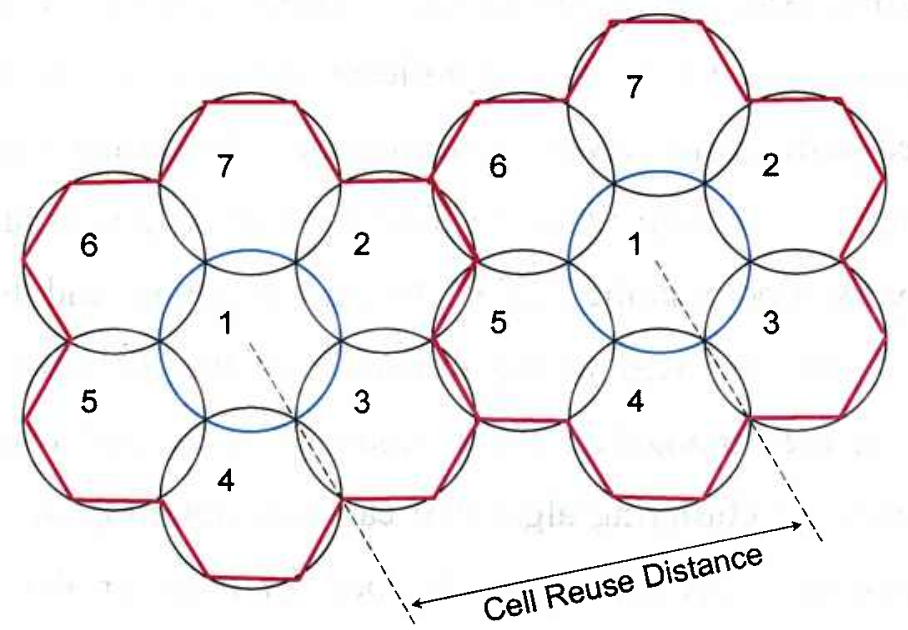

Figure 3-2 Allocation of $P N$ codes in a $K=7$ cell reuse pattern. The number assigned to each cell represents the specific PN code that nodes can use to communicate in the colocated geogrpahic area. PN codes are reused as the cell clusters are adjoined to cover a larger geographic area. The cell reuse distance is the minimum distance between cells using the same PN code. 
pattern and the fewer PN codes required.

The first step in determining cluster size is to formulate an appropriate transmission loss model between the source and its receiver in a shallow water environment. Using fundamental equations from acoustic wave theory, the range and frequency dependent transmission loss $(H)$ between a source and its receiver is approximated by

$$
H=10 \log r+10 \log (\min \{h, r\}) \cos \theta_{i}+\alpha r
$$

where $r$ is the range between the source and the receiver, $h$ is the total water depth, and $\theta_{i}$ is the minimum incident angle between the dominant eigenray and a vertical channel boundary [21]. The first terms represents cylindrical spreading losses out to the receiver with the second term accounting for spherical spreading losses up to a range equivalent to the total water depth. All calculated quantities of sound pressure are given in units of $\mathrm{dB}$ referenced to $1 \mathrm{uP}$ at 1 meter unless otherwise stated. Assuming a constant transmitter power $\left(P_{T}\right)$, the logarithmic signal strength at the receiver $(S)$ is given by

$$
S=10 \log \left(P_{T}\right)+171-H
$$

where the additional factor of 171 is used to convert the logarithmic expression into decibel units referenced to $1 \mu \mathrm{Pa}$ at 1 meter. The next step is to quantify the level of interference at the receiver from other nodes within the network. The magnitude of interference received depends on the PN code and the relative proximity between the receiver and the interferers. The co-channel interferer nodes that simultaneously transmit using the same PN code as the source-receiver pair in question must be sufficiently separated to prevent mutual interference. As discussed in section 2.2, adjacent channel interferer nodes that transmit using a different PN code than that of the source-receiver pair produce little more that wideband noise at the receiver. The relative magnitude of this noise depends on the proximity of the adjacent node and 
processing gain of the spread spectrum acoustic modem. If the adjacent node is too close, the receiver can be completely saturated and unable to decode the intended signal. In traditional cellular theory, this phenomenon is known as the near-far problem, and is typically minimized using coordinated power control techniques between the base station tower and the mobile user. Due to the nature of the UACC and the performance characteristics of the transducers used in acoustic modems this problem is very difficult to overcome by just power control. Thus, sufficient separation between nodes using different PN codes is still required to provide the minimum SIR to the modem's receiver.

In order to calculate the total interference from co-channel and adjacent channel interferers at the receiver a cell reuse pattern must be assumed. Using the cell geometry, the worst-case distances between the receiver and the interferers can then be estimated and applied to the transmission loss model for receiver SIR calculations. Traditional cellular theory textbooks provide convenient lookup tables that show the cell reuse distances as a function of cluster size. These values are based on a mobile cellular system that has fixed towers located in the center of the cell. The design equation used in their calculation must now be altered to accommodate the movement of both the user and the tower (user). The worst-case arrangement of the transmitter, receiver and interferers (co-channel and adjacent) for the distributed cellular network in a $\mathrm{K}=7$ cell reuse pattern can be see in Figure 3-3. Each cell cluster is replicated using Dikstra's minimum distance algorithm to produce a super-cluster of 7 cell clusters. Using the above cell configuration the SIR at the receiver can be calculated using the method shown in section 2.3. Let $d_{c o, i}$ denote the distance between the receiver and the $i^{\text {th }}$ co-channel interferer. Then the interference at the receiver due to all co-channel interferers $\left(I_{C O}\right)$ is calculated as

$$
I_{C O}=10 \log \left[\sum_{i} P_{T}\left(d_{c o, i} \min \left\{h, d_{c o, i}\right\} \cos \theta_{i} 10^{\frac{\alpha d_{c o, i}}{10}}\right)^{-1}\right]
$$



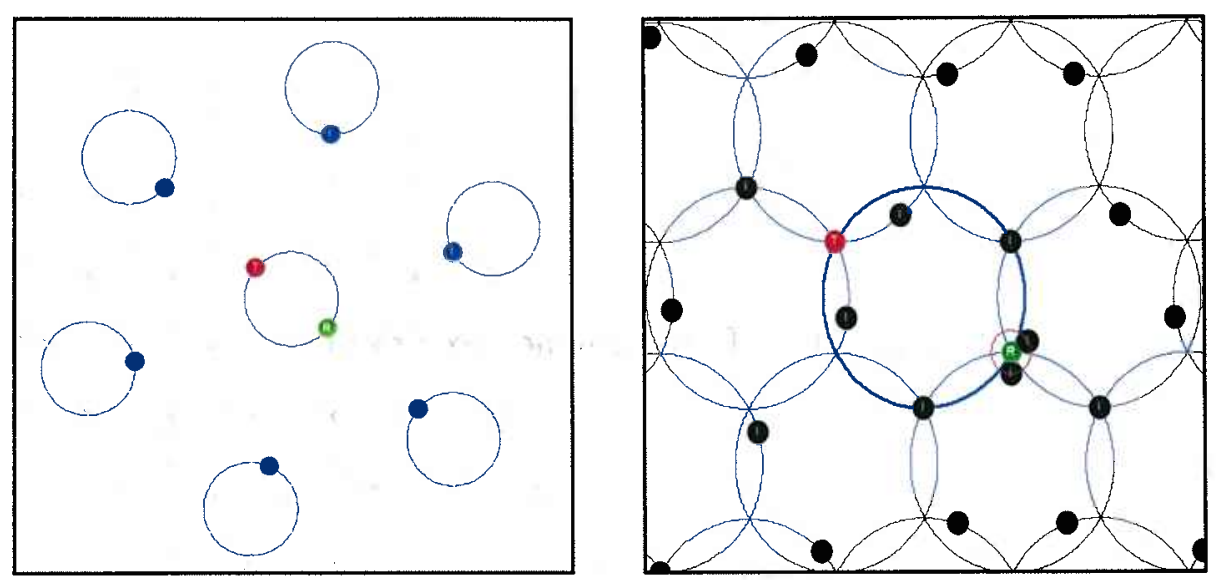

Figure 3-3 Transmitter (red), receiver (green), co-channel interferers (blue), and adjacent channel interferers (black) in a distributed cellular network

A similar expression for the interference at the receiver due to all adjacent channel interferers $\left(I_{A D J}\right)$ can be found using equation (3.3). As previously stated, the total interference from adjacent channel nodes is reduced by a factor equivalent to the processing gain. Finally, the SIR at the receiver is given by

$$
S I R=S-I_{C O}-I_{A D J}+P G
$$

A detailed examination into the effects of various cell reuse patterns and modem processing gains on receiver SIR will be presented in chapter 4 .

\subsubsection{Node Clustering and Cell Orientation}

While mapping nodes into geographically fixed cells can provide localized control of communication resources, it prohibits nodes located in one cell from communicating with nodes located outside its cell coverage area. As an example, if a pair of mobile nodes communicating within a given cell maneuver such that they find themselves in different cells, then the communications link will no longer exist. More importantly, how does a node route a message across the network to a more remote node, such as an information gateway? Therefore provisions for allowing 
adjacent nodes to communicate across cell boundaries must be included in the distributed cellular clustering scheme.

Using the assumption that nodes can fix their positions in time and the parameters that define the geometry of the cellular network are known, then nodes can recreate the entire cell pattern relative to any fixed point in the UACC, map themselves to these cells, and determine the specific communication resources available. Extending this concept further, if the reference point that fixes the cell pattern geographically is shifted slightly to an alternate position then new node clusters can be formed between previously adjacent nodes. If this process is periodically repeated using a predefined pattern, then the overall effect results in the ability of adjacent nodes to communicate with each other over an area greater than
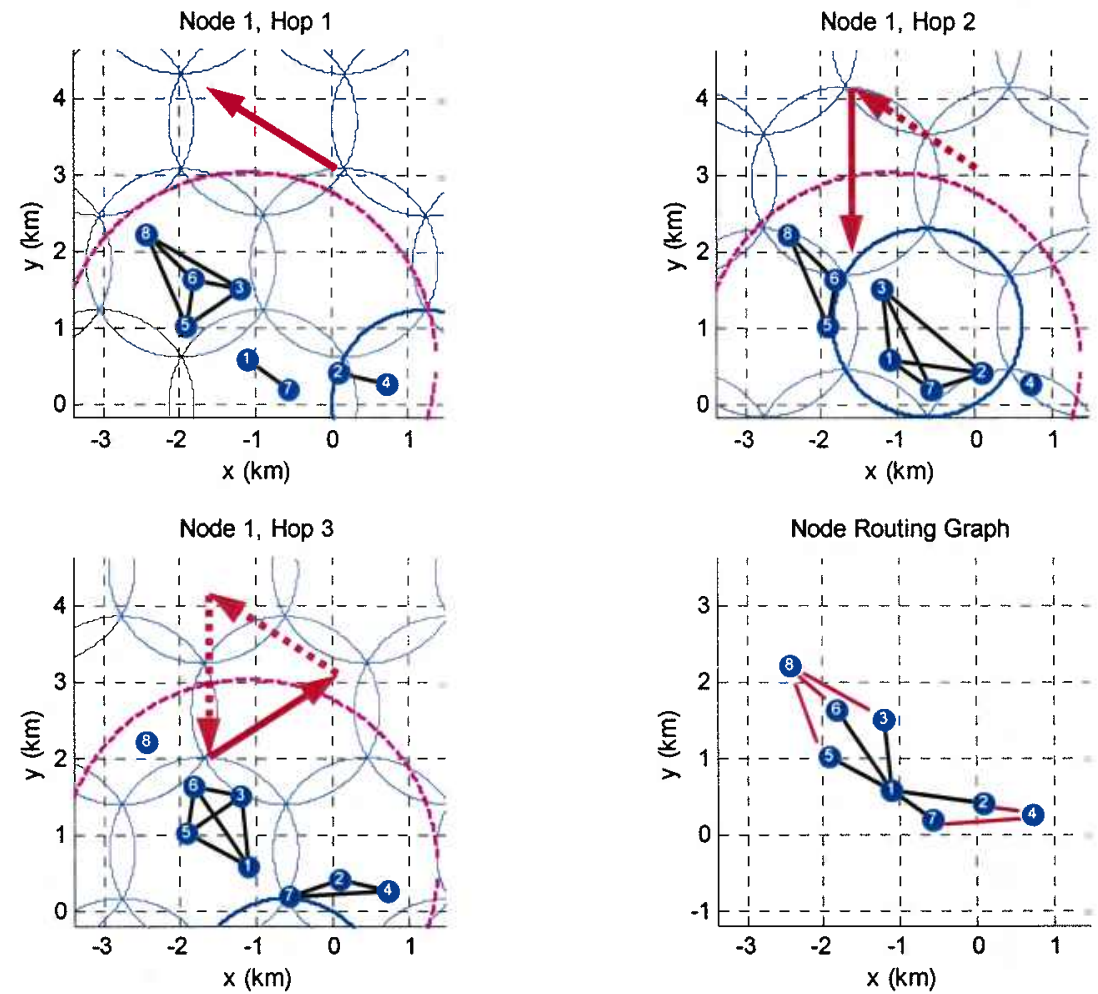

Figure 3-4 Demonstration of a full hop cycle and the resultant network routing graph for Node 1. The solid red arrows indicate the next hop point. The red ares in the Node Routing Graph indicate available 2 hop paths to distant nodes. 
the cell size. This process of shifting, or hopping, the cell pattern in position and time is the underlying concept in the spatially dynamic cellular clustering scheme presented in this thesis. Figure 3-4 illustrates how spatially dynamic cellular clustering provides network connectivity between Node 1 and its nearest neighbors. The black arcs between the nodes indicate the adjoining nodes have been clustered together within the co-located cell. The hopping algorithm follows a predefined pattern (shown in red) that pauses at each point to allow the distributed clustering algorithm to group nodes based on their relative positions to the cell pattern and each other. Once the nodes have been clustered and their cell membership determined, the nodes can then communicate using the PN code designated for their assigned cell. It is at this point nodes share access to the communication channel in each individual cell by implementing the prescribed MAC protocol. The length of time spent at each hop point to allow communications between nodes to occur is a fixed quantity defined in the configuration parameters of the protocol. Once the predefined time expires, the protocol hops to the next point in the cycle to allow reclustering. Upon completion of the entire hop cycle, Node 1 has been able to communicate directly with every node except nodes 4 and 8 , as shown in Node Routing Graph. However, node 1 can still pass traffic to nodes 4 and 8 using the following multihop paths during the hop cycle:

$$
\begin{array}{cc}
\frac{1 \rightarrow 4}{1 \rightarrow 2 \rightarrow 4} & \frac{1 \rightarrow 8}{1 \rightarrow 3 \rightarrow 8} \\
1 \rightarrow 7 \rightarrow 4 & 1 \rightarrow 5 \rightarrow 8 \\
& 1 \rightarrow 6 \rightarrow 8
\end{array}
$$

\subsection{Distributed Clustering Algorithm}

The spatially dynamic cellular clustering algorithm allocates communication resources and shapes the network structure by clustering nodes together based on their geographic positions. An important aspect of the clustering algorithm is its 
ability to converge to the same network structure independent of which node executes the algorithm using only the positional information of nearest neighbor nodes. First, the global position of each cell is converted to a cellular coordinate position relative to the network reference coordinate. Nodes are then mapped to the network cells that overlap with their positions. Those nodes that are within close proximity to one another are preferentially clustered to minimize the effects of the near-far problem and maximize the SIR at the receiver. Next, nodes that have been mapped into multiple cells are dynamically clustered to reduce the membership of each node to only one cell. Nodes clustered into a cell are then permitted to communicate by sharing the geographically allocated PN code for a predetermined period of time. Finally, the positions of the cell boundaries are reoriented and the clustering process described above is repeated.

The distributed cellular clustering algorithm can be decomposed into three separate algorithms that are iterated in sequential order: cell hopping, cell membership, and node cell clustering. The following subsections will provide further detail into the inner workings of these algorithms. In chapter 4 , the effect of these algorithms and their associated parameters on network connectivity, SIR, and scalability will be examined quantitatively though simulation.

\subsubsection{Cell Hopping Algorithm}

The cell hopping algorithm (Cell_Hop) is responsible for controlling the periodic repositioning of cell boundaries in position and time by adding a position offset value to a fixed reference coordinate in the UAN, then publishing the resultant northing and easting positions to the subordinate algorithms. Nodes can then recreate the cell pattern relative to the new network reference coordinate and recluster nodes accordingly. 


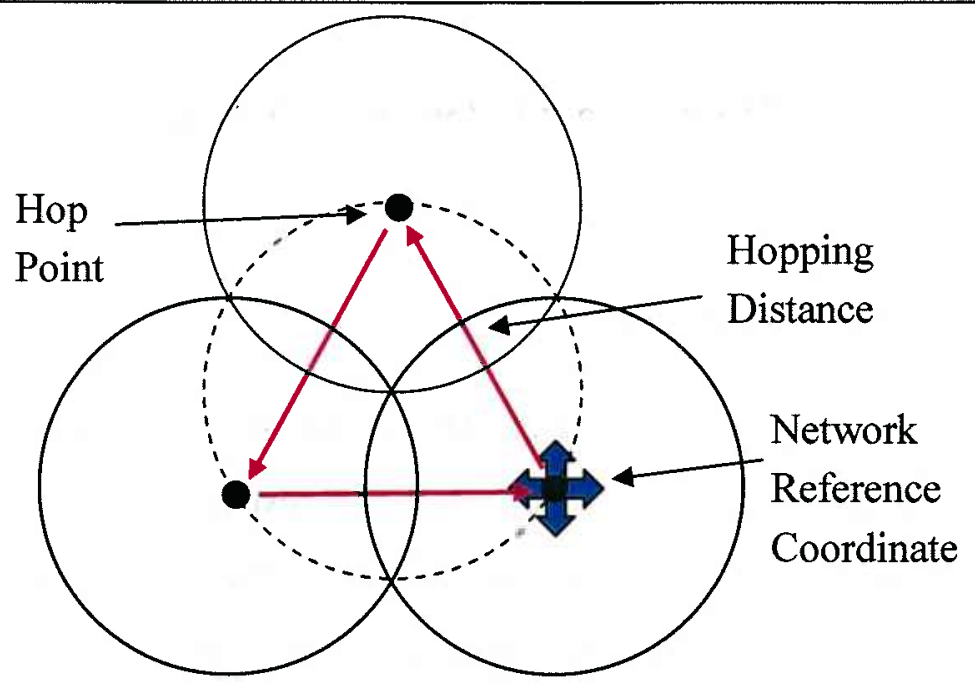

Figure 3-5 Cell hopping geometry

The algorithm requires the following network configuration parameters for proper execution:

1. Network reference coordinate (net_coor), expressed in northing and easting

2. Cell radius (cell_radius), expressed in meters

3. Number of hopping points (hop_points)

4. Hopping distance (hop_dist), expressed in meters

5. Hopping period (hop_period), expressed in seconds per hop

Figure 3-5 is an illustrative example of the time dependent cell hopping geometry for a 3 hop distributed cellular system. In the illustration, the origin of the cell centered on the network reference coordinate follows the hop points in a counter-clockwise rotation about vertices of the red equilateral triangle. Using the time-dependent position of this cell as a reference point, nodes can then triangulate the relative cellular coordinate position of every other cell in the network. The Cell_Hop parameters listed above must be predefined in a network configuration file that can be referenced by the distributed clustering algorithm prior to network deployment. It is important that each node is furnished with the same configuration parameters to 
prevent network conflicts. The psuedocode for the Cell_Hop algorithm can be found in appendix A.1.

\subsubsection{Cell Membership Algorithm}

The cell membership algorithm (Cell_Membership) takes as an input the coordinate position of the host node and all of its nearest neighbor nodes in the NODEPOSIT table, compares their positions to the current network reference coordinate, and then maps them to the appropriate cell(s). Depending on a node's proximity to a cell boundary, it may be mapped into two overlapping cells.

The first step in mapping a node to a cell is to calculate its cellular coordinate position relative to the network reference coordinate. Using Figure 3-6, this coordinate is calculated with the following steps:

1. Using a line with a slope of $\arctan (\pi / 3)$ extending from the node's UTM position calculate the easting component of the $i$ axis intercept point $\left(\mathrm{L}_{\mathrm{i}}\right)$ (vertex of red arrows). This length is proportional to the $i$ component of the cellular coordinate.

2. Calculate the straight-line distance between the $i$ axis intercept point at the node's UTM position $\left(\mathrm{L}_{\mathrm{j}}\right)$. This length is proportional to the $j$ component of the cellular coordinate.

3. Normalize $L_{i}$ and $L_{j}$ by the distance between the centers of two adjacent cells, then round down to their integer value to get the actual cellular coordinate $(i, j)$ of the node. 


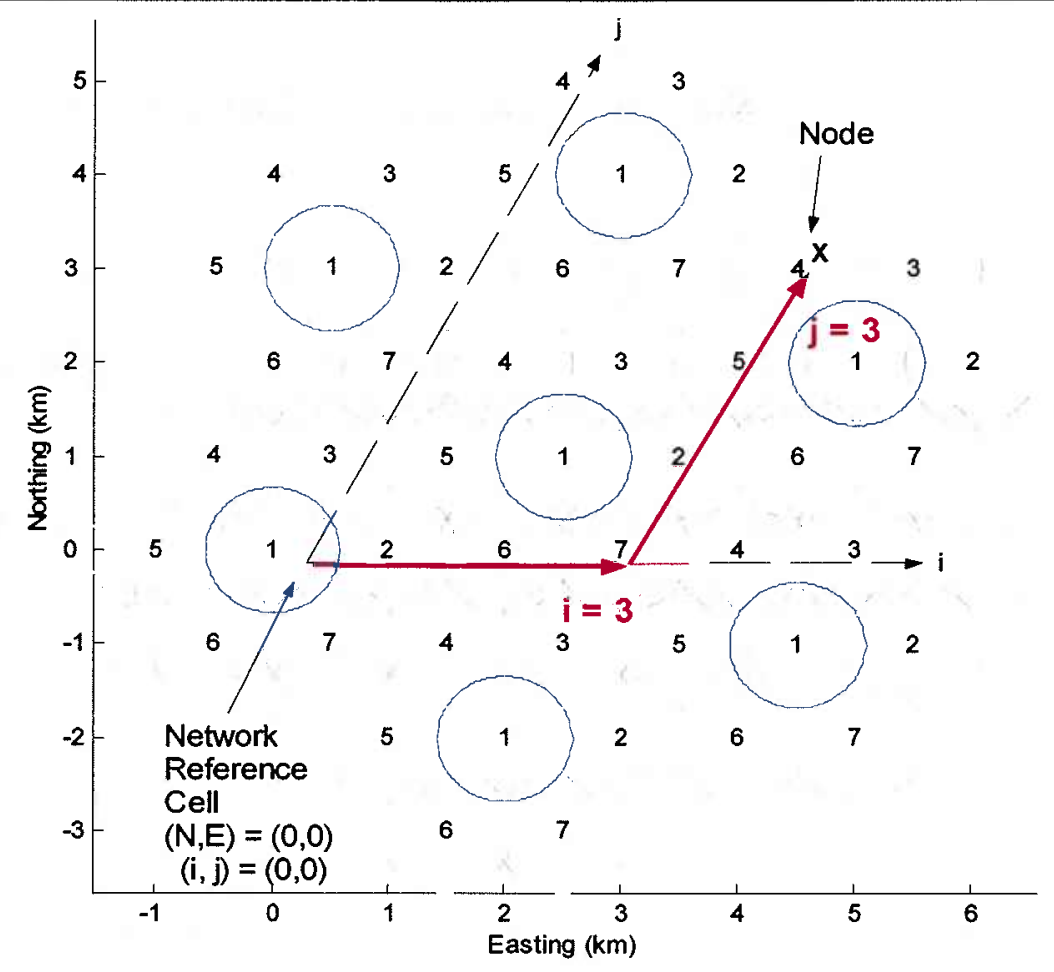

Figure 3-6 Determination of cell membership in a $K=7$ cell reuse pattern

Since the distributed cellular network consists of repeating clusters of geometrically identical cells, the actual cell number, in terms of the $i$ and $j$ cellular coordinate positions shown in Figure 3-6, are also repeating patterns of cell numbers. The cell centered on the network reference coordinate is always labeled as the first cell (cell 1 ) in the cell reuse pattern. The sequential pattern of cells extending in the $i$ and $j$ directions from this cell are known as the ihop_cell and jhop_cell vectors, and have the following values for $\mathrm{a}=7$ cell reuse pattern shown in Figure 3-6:

$$
\begin{aligned}
& \text { ihop_cell }=\left[\begin{array}{lllllll}
1 & 2 & 6 & 7 & 4 & 3 & 5
\end{array}\right] \\
& \text { jhop_cell }=\left[\begin{array}{lllllll}
1 & 3 & 7 & 2 & 5 & 4 & 6
\end{array}\right]
\end{aligned}
$$

By inspection of Figure 3-6, the node's cell number for position $(3,3)$ is 4 . More generally speaking, count 3 cells in the positive $\mathrm{i}$-axis direction from the Network Reference Cell to arrive at cell 7 . From this cell, count 3 cells in the positive j-axis direction to arrive at cell 4 . The following algorithm outline how the cell number 
can be determined with the $(i, j)$ cellular coordinate using modulo $\mathrm{K}$ math $(\%)$ and the ihop_cell and jhop_cell vectors:

1. Determine the $i$ axis intercept cell number: i_cell $=$ ihop_cell $(i \% \mathrm{~K})$

2. Starting at the jhop_cell index value corresponding to i_cell, rotate right through the jhop_cell vector $j$ steps to find the actual cell number

The UTM coordinate position of the calculated cell and its first ring cells are then compared to the UTM coordinate position of the node in question. Any cell within a distance less than or equal to a cell radius of the node will be mapped to that node.

The host node executing the Cell_Membership algorithm will iterate through all of the nodes within its NODEPOSIT table to generate an incidence matrix of node to cell membership mappings called the nodecell_map. The matrix will be used by the distributed clustering algorithm to optimize and finalize the cell membership of each node within the NODEPOSIT table. The rows of the nodecell_map matrix correspond to the node number while the columns correspond to the 1 through $\mathrm{K}$ possible cell numbers in the given cell reuse pattern. The distance between the centers of the host node cell and the neighbor node cells will be compared to prevent nodes located within a more distant cell cluster from being aliased into the

\begin{tabular}{|c|c|c|c|c|c|c|c|c|}
\hline & \multicolumn{7}{|c|}{ Cells } \\
\hline & & 1 & 2 & 3 & 4 & 5 & 6 & 7 \\
\hline \multirow{6}{*}{ Nodes } & 2 & 0 & 0 & 1 & 0 & 0 & 0 & $\overline{0}$ \\
\hline & 3 & 0 & 0 & 0 & 1 & 0 & 0 & 0 \\
\hline & 5 & 0 & 0 & 1 & 0 & 1 & 0 & 0 \\
\hline & 6 & 0 & 0 & 0 & 1 & 0 & 0 & 0 \\
\hline & 8 & 0 & 0 & 1 & 0 & 0 & 0 & 0 \\
\hline & 9 & 0 & 0 & 1 & 0 & 0 & 0 & 0 \\
\hline
\end{tabular}

Figure 3-7 Node cell membership matrix for 6 node in a $K=7$ cell reuse pattern 
nodecell_map. Those node with center cell distances that exceed the radius of the first ring cells in the given cell reuse pattern are excluded from the mapping. Figure 3-7 shows an example nodecell_map for 6 nodes in a $K=7$ cell reuse pattern. A matrix entry of " 1 " indicates that the node (row) is mapped to the corresponding cell (column). The pseudo-code for the Cell_Membership algorithm can be found in Appendix A.2.

\subsubsection{Node Cell Clustering}

While the Cell_Hop and Cell_Membership algorithms deterministically map the coordinate positions of nodes into time-dependent, geographically separated cells it is the node cell clustering algorithm (NodeCell_Cluster) that actually optimizes and finalizes the network structure in a distributive manner to meet the following criteria at each node:

1. Maximize the SIR at the receiver

2. Maximize network connectivity

3. Maximize the use of available communication resources

It is at this point the spatially dynamic cellular clustering algorithm can begin to organize the positional (NODEPOSIT) and cell membership (nodecell_map) information of the host node into an efficient distributed network structure.

\section{Interrelationships between Cluster Performance Criteria}

Before the process of optimizing the network structure can begin it is important to understand the significance of each of the above listed criteria to prevent potential conflicts during optimization. As previously discussed, nodes that are within close proximity of one another in a CDMA network must overcome the limiting effects of the near-far problem. One method presented was the use of power control techniques at the transmitter. However, this method may not be available in modems 


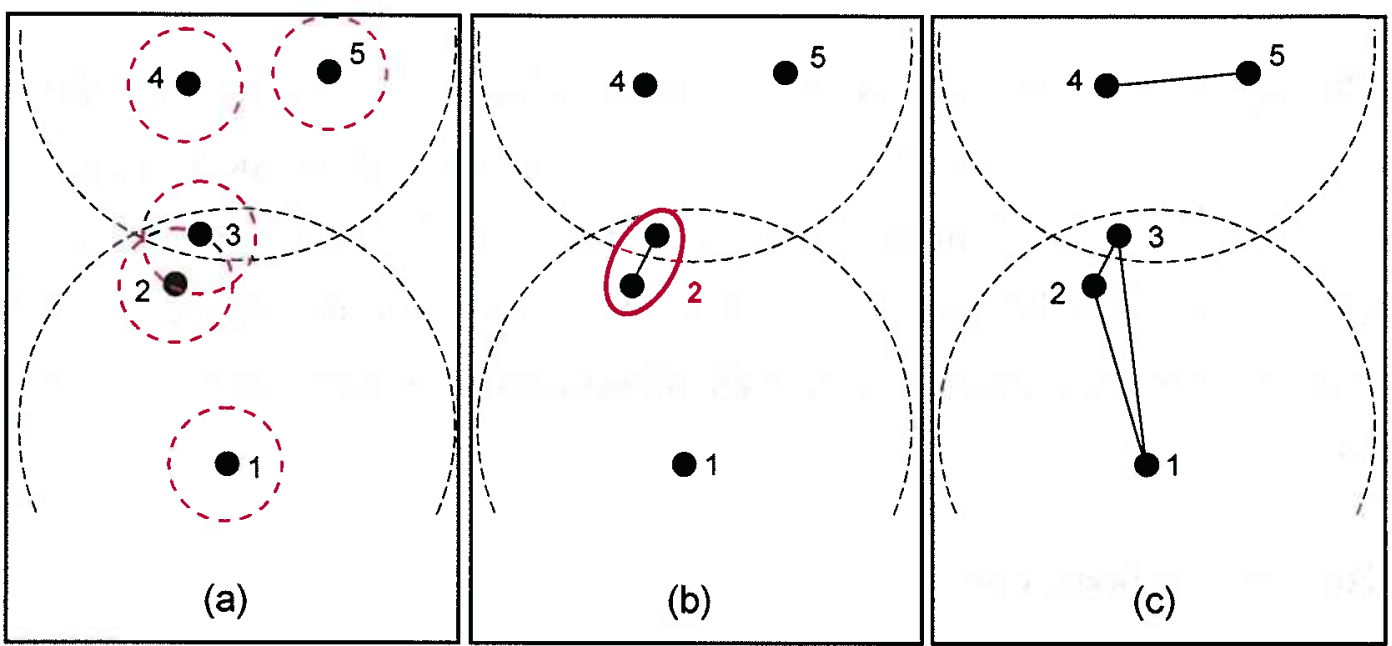

Figure 3-8 Minimizing the effects of the near-far problem with pre-clustering. (a) The distributed clustering algorithm compares the range proximity between each pair of nodes in the network to determine if a potential near-far problem exists. (b) Those nodes that are within too close of a proximity to one another are pre-clustered together as a supernode (red ring). (c) The clustering algorithm will uses these supernodes to then cluster nodes accoringly.

with a fixed transmitter power. Another method that requires no additional equipment functionality is to pre-cluster nodes within close proximity of one another into the same cell, creating a super-node as shown in Figure 3-8. This would prevent those nodes most vulnerable to the near-far problem from interfering with one another by forcing them to share the same communication resources. Although this effectively mitigates the near-far problem, it introduces another problem: communication resource allocation. Using the pre-clustering technique now forces more nodes to share the already limited communication resources between a larger set of nodes in a cell. Consequently, as the cluster grows larger the network's performance in terms of throughput and delay will begin to degrade. Thus, the clustering algorithm must strike a balance between the contradicting criteria of maximizing the SIR at the receiver and maximizing available communication resources.

The criterion of maximizing network connectivity is slightly less limiting in relation to SIR and communication resources. In fact, pre-clustering nodes enhances 
Distributed Clustering Algorithm

network connectivity while communication resource allocation has little effect. The only limiting case for communication resource allocation that tends to degrade network connectivity is in the access-limited case where too many nodes attempt to share the channel in a time period much less than can be accommodated by the MAC protocol [5]. In this case, concessions can be made to improve performance by adjusting the communications time to meet the requirements of MAC protocol or vice-versa.

\section{Addressing the Near-Far Problem}

After reflecting on the interrelationships between the criteria above it seems more appropriate to address the near-far problem than communication resource allocation since the spread spectrum communication modems employed in UANs are more limited by receiver SIR [1, 16]. Communication resource allocation is more application specific (e.g., telemetry versus video) and highly dependent on traffic routing protocols, which is an aspect of UAN design that needs further development. As such, the distributed clustering algorithm developed here will strive to maximize receiver SIR.

Using traditional cellular system theory, a natural starting point in defining the minimum range limit, or proximity limit (prox_limit), between adjacent nodes is in the region of cell overlap called the handoff region. It is in this region that CDMA cellular systems force users to transition from using the resources in one cell to those allocated in the adjoining cell before the call is dropped. In the distributed cellular system, it is the nodes within the handoff region that are more prone to the near-far problem due to the geometrical boundaries of the cell structure. A pair of nodes located in the cell overlap region that are afflicted by the near-far problem can be easily clustered into one of the overlapping cell. If the nodes are in strictly different cells, then the actual proximity limit must now take into consideration the ability of a distant node at the farthest reaches of the cell to communicate with the node beyond 
the PDR90 of the modem. Thus, a reasonable compromise is to set the prox_limit to equal the maximum overlap distance between 2 adjacent cells. Using geometry and the PRD90 of the modem the prox_limit is given by

$$
\text { prox_limit }=2\left(1-\cos \frac{\pi}{3}\right) P R D 90
$$

Simply stated, nodes with relative proximities that are within approximately $27 \%$ of the PRD90 of the acoustic modem will be pre-clustered into the same cell to mitigate the effects of the near-far problem. During the cluster optimization phase, the protocol will treat these nodes as a super-node using the node cell mapping data of the lower numbered node to perform the optimization.

\section{Optimizing Network Structure}

After pre-clustering, the nodecell_map now contains all of the information needed by the distributed clustering algorithm in optimizing the network structure. The goal in this final phase of the algorithm is to cluster nodes into cells such that the distance between clustered nodes is minimized. At the same time, the algorithm will insure that no nodes are left unclustered or belonging to more than one cell. The pseudocode for the NodeCell_Cluster algorithm can be found in appendix A.3

The optimization algorithm is an iterative process that ends when the cell membership of each node in the nodecell_map corresponds to exactly one cell. Starting with the nodes that have the lowest cell membership, the algorithm will systematically search for and cluster each of these nodes, called object nodes, with any other nearest neighbor node, called the target node, provided the following two requirements are met:

1. The cell membership of the object node and the target node overlap in one or more cells. 
2. The arc to the target node is a minimum distance arc that is fully contained within the boundaries of a cell.

Once the object node has been clustered to an appropriate target node, the mutually inclusive cell membership data the two will be substituted back into the nodecell_map under the index of the lower numbered node. The process will then be repeated for those object nodes that have the next highest cell membership, and so on. Finally, the optimized nodecell_map will contain all the communication resource allocation information required by the host node executing the distributed algorithm. Nodes clustered under one particular cell in the nodecell_map will communicate using the PN code specified for that cell. This information is crucial for sharing the cell channel among the similarly clustered node using the prescribed MAC protocol and in determining the available routing path for traffic between nodes.

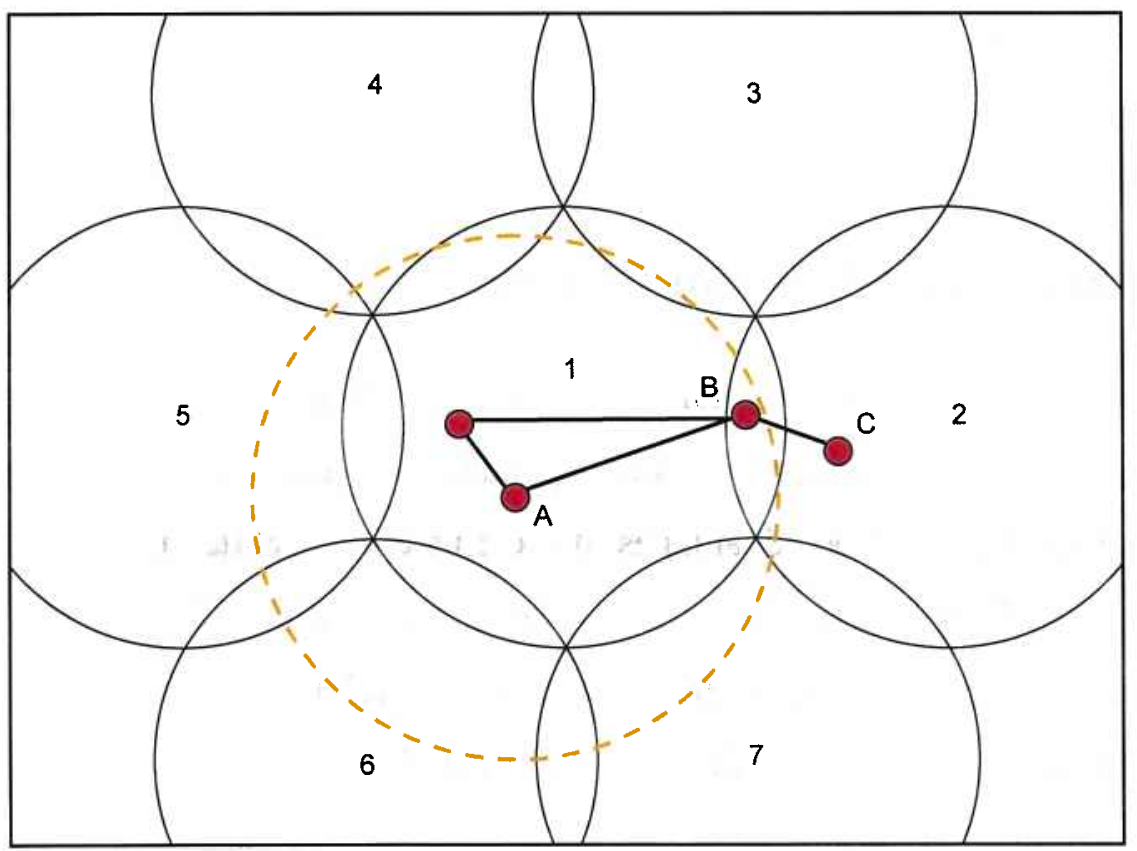

Figure 3-9 Example of a node clustering conflict. The position of th nodes within the orange circle are know to node $A$ 
Assuming each node within the network has a priori knowledge of their nearest neighbor node positions, each node should then be able to generate a nodecell_map using the NodeCell_Cluster algorithm that is nearly identical to one another. The minor differences that can occur between these mappings stems from the fact that each node does not have complete knowledge of every node within the network. Figure 3-9 illustrates an example of this potential clustering conflict. In the example, node A may preferentially cluster the more distant node B in an effort to reduce the likelihood of it being alienated. At the same time, node B may have chosen not to cluster node A, but instead cluster the much closer node $C$. Under these circumstances, the nodes clustered in cell 1 with node A would attempt to communicate with node B without success in this hop. The process of relocating the cell boundaries and re-clustering the nodes increases the probability of node $\mathrm{B}$ being clustered with node $\mathrm{A}$ at some point in the hop cycle. The MAC protocol must address the issue of false cluster membership, as shown above, using either reservations or a node discovery process.

\subsection{Medium Access Control Protocol}

The discussion in this section seeks to offer a generalized approach to implementing a MAC protocol in the spatially dynamic cellular clustering architecture. One major of the advantages of the clustering architecture is its ability to partition a large field of nodes into smaller, more manageable cells of nodes. This approach can allow UAN designers the flexibility to implement a significantly less complex MAC protocol in each individual cell of nodes vice coordinating channel access over the entire network.

The overall performance of a MAC protocol is typically gauged by its throughput efficiency and delay characteristics. Throughput efficiency is the measure 
of how much of the communications time is spent transmitting user data over a given period of time, while delay is the measure of how long it takes before the data can be transmitted. More directly, the characteristics of an efficient MAC protocol are its ability to quickly free up communication resources and minimize the time wasted between transmissions. Along this same line, the quicker a user can send its packet to the destination, the soon someone else can reuse the channel. The ratio between the time it takes a signal to propagate though the medium $(\tau)$ to the transmission time of a packet $\left(D_{T P}\right)$ is called the normalized propagation delay $(a)$.

$$
a=\frac{\tau}{D_{T P}}
$$

In general, the normalized propagation delay found in modern cellular phone systems and LANs is somewhere between $10^{-7}$ and $10^{-3}$. Comparatively, the relatively low data rates of acoustic modems coupled with the inordinately large propagation delay of sound in the UACC produces a normalized propagation delay between $10^{-2}$ and $10^{2}$ ! One way to enhance the efficiency of a MAC protocol in an UAN is to minimize the amount of network management overhead in packet transmissions and minimize the time wasted between data transmissions from changeover. Network management overhead is defined as any transmission, or part of a transmission that is responsible for coordinating resource allocation, network configuration, or message routing. The less complex the network architecture, the less network management overhead required the more time spent exchanging user data and the higher the network efficiency. Changeover is defined as the time it takes to free up the communications channel between two users. Possible sources of this delay are the time it takes for the communications hardware to reverse from "receive" to "transmit", polling nodes for traffic, or the inclusion of a specified guard time between users to prevent potential transmission overlaps. While these delays 
may be small, the more users that are added the more changeover delay introduced and the longer a user must wait between transmissions.

The spatially dynamic cellular clustering algorithm strives to reduce the complexity of network management by geographical allocating communication resources into cells of co-located nodes. This method limits transmission overhead and enhances network efficiency by reducing number of transmissions required to coordinate resource allocation. Since the network structure and communication resources are known, implementing the MAC protocol is now a matter of providing fair, efficient access among an exclusive group of nodes. A straight-forward MAC protocol that can be easily integrated into the distributed cellular architecture is TDMA.

Implementing the TDMA protocol is a rather straight-forward process. The communications time, or frame time, at each hop point in the cycle can be divided into smaller, equally spaced time slots based on the number of nodes clustered in the cell. Nodes are assigned a dedicated time slot within the frame to transmit there traffic according to their assigned node number. The transmission priority of each node is assigned using the node cell membership information from the nodecell_map. The node with the highest priority (lowest node number) will transmit first, followed by the next lower priority node, and so on. While this approach is straight forward, it can become quickly inefficient in the case where clustering conflicts occur. TDMA slots would stay idle for those nodes that were allocated slots, but were falsely clustered into the cell. A method of validating the actual cell membership at the beginning of each frame is required to further increase efficiency. On method is to allocate a slot for each node to explicitly report its membership in the cell, much like a reservation request. A node wishing to guarantee a time slot for passing its traffic in the cell cluster will transmit a reservation request message during the first time slot that it is allocated in the frame. 


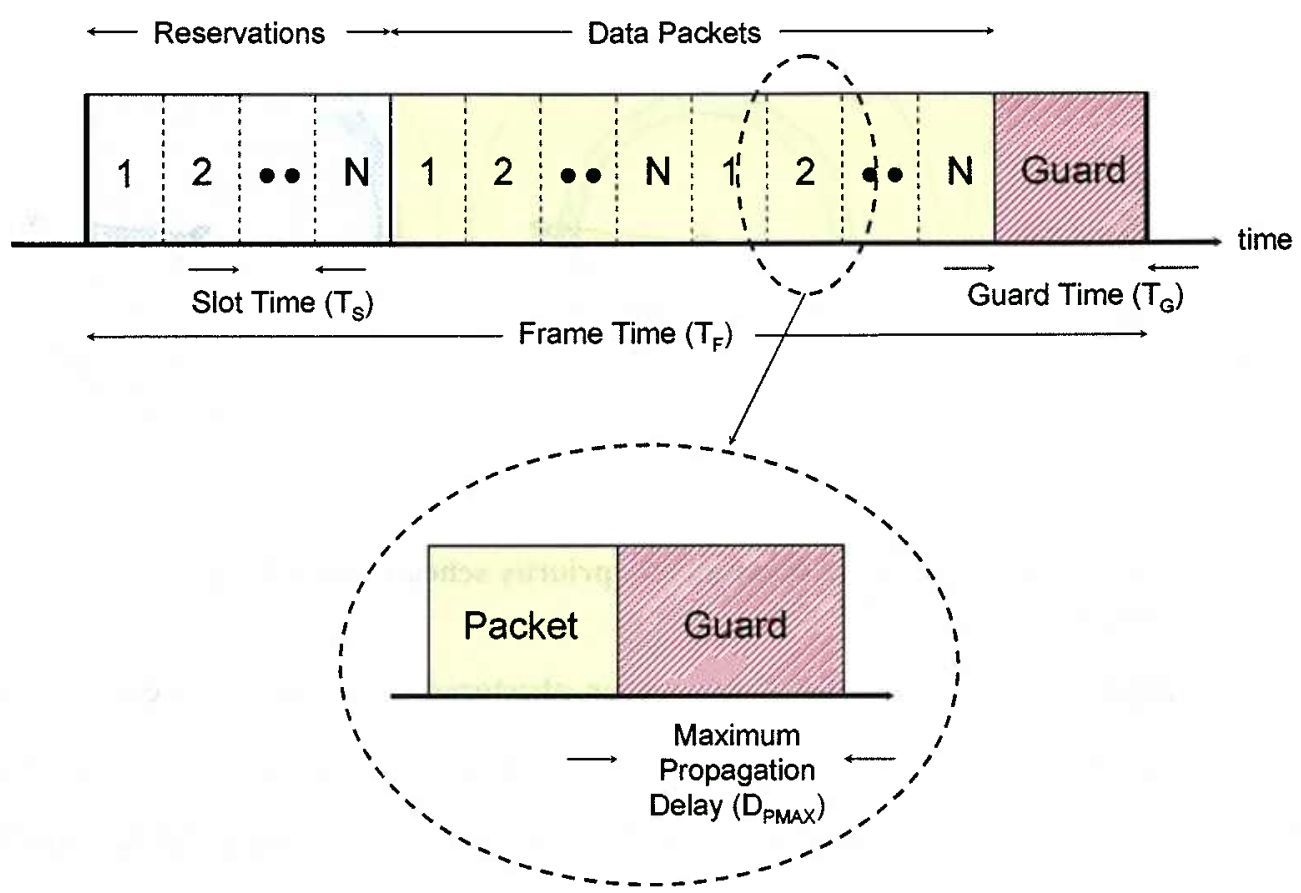

Figure 3-10 TDMA with reservations timing diagram. The maximum propagation delay guard time used in UANs consumes a significant portion of the total slot time

The length of the reservation time slot can be either the same size as a full length data slot or smaller depending on the packet sizes made available by the modem. A timing diagram showing the order of reservations and data packets slots in a TDMA reservation protocol can be seen in Figure 3-10. Using these requests nodes can either allocate future slots accordingly or absorb and redistribute those slots that would otherwise remain idle. In addition, the reservation request information can be exploited by a routing algorithm for the validation of traffic routing paths.

The total slot time $\left(\mathrm{T}_{\mathrm{S}}\right)$ allocated to each node is the sum of both the packet transmission time $\left(\mathrm{D}_{\mathrm{TP}}\right)$ and the maximum propagation delay guard time (DPMAX.) Depending on the number of nodes per cell a significant portion of the already limited communication resources can be wasted while waiting for packets to propagate through the channel. Using a transmission priority scheme, a more desirable approach would be for the next higher priority node to transmit once it receives the packet of the highest priority node. Figure 3-11 shows how this 


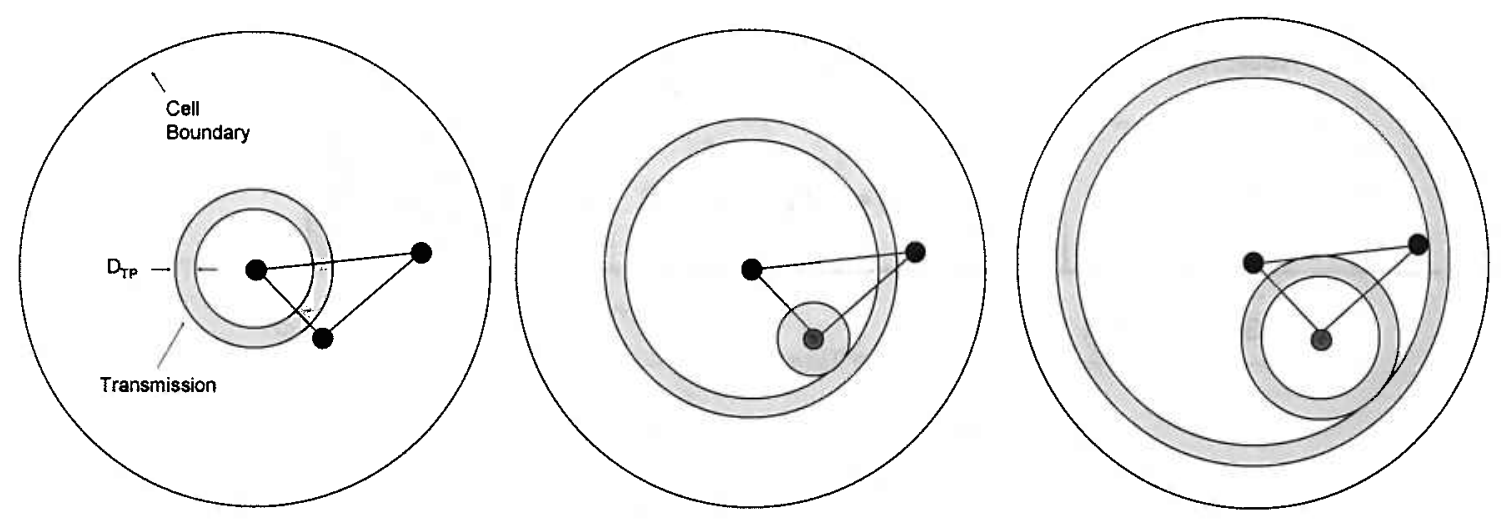

Figure 3-11 Diagram of how successive transmission priority scheme can reduce the time wasted from waiting for the channel to clear.

transmission scheme would work for three nodes clustered together in a cell. While this method does not completely eliminate the propagation delay, it does shorten it by a length proportional to the distance between the two nodes. Depending on the positions of the nodes in the cell a significant portion of the frame time can be reclaimed and reused by the nodes instead of being wasted while waiting for the channel to clear.

Since the boundaries of the cellular clustering scheme reposition in time there is the potential that the last transmission from a frame will still exist at the next hop point. The decaying transmission would interfere with the new clustering of nodes resulting in a potential breakdown of the priority transmission scheme. In order to prevent this situation from occurring a guard time $\left(T_{G}\right)$ is inserted at the end of the frame to ensure that the last packet transmitted in the frame will have sufficient time to clear the channel at the next hop point. The total guard time required is the sum of the propagation delay in traversing the diameter of a cell $\left(D_{C E L L}\right)$, the propagation delay incurred in clearing the additional hopping distance of the cell pattern $\left(D_{H O P}\right)$, and the maximum delay spread of the channel $\left(D_{S P R E A D}\right)$.

$$
T_{G}=D_{C E L L}+D_{H O P}+D_{S P R E A D}
$$


While the transmission scheme reduces the transmission delay between successive priority nodes, it also introduces problems with clustering conflicts and slot synchronization. If a falsely clustered node never transmits, then when is the next node supposed to transmit? One approach is to set a safety period between successive transmissions such that if a node never transmits, then the next higher priority node can transmit after the safety period has expired. In this way slots are reserved implicitly by either a transmission (reservation) or silence (no reservation.) Minislotted Alternating Priorities (MSAP) is a demand assignment multiple access protocol that uses the implicit reservation scheme described above to provide efficient channel access to a prioritized grouping of nodes [7]. In MSAP, the frame time is subdivided into much smaller minislots that are equal to the maximum propagation delay between any two nodes. In the case of the distributed cellular system, the length of the minislot is proportional to the diameter of a cell. The spacing ensures that any node within the channel will be able to hear the start of a transmission prior to the beginning of the next minislot. Nodes transmit packets at

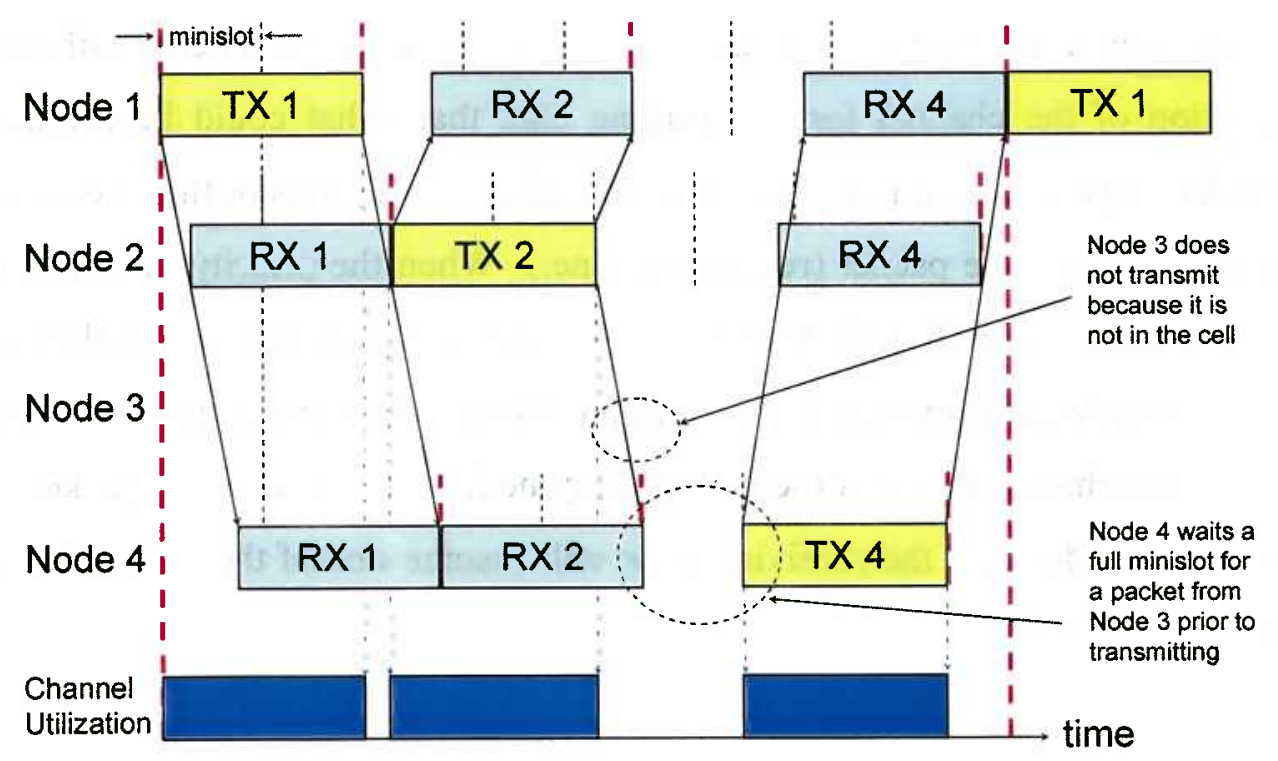

Figure 3-12 Transmission timing diagram for a distributed cellular UAN of 4 nodes using a hybrid MSAP MAC protocol. The red dashed lines indicate the reset point of the minislot timing at each node for a significant transmission event 
the beginning of a minislot based on their transmission priority. Simultaneously, all other nodes within the cell will listen to the channel to determine whether the priority node has either transmitted a packet or deferred. If the start of the transmission from the priority node has not been received by the end of the assigned minislot, then the next higher priority node will begin its transmission at the beginning of the next minislot. In this way, the reservation request (cell membership) of the priority node is implied to all by either receiving the start of a packet (member) or the passing of an empty minislot (non-member.) The MSAP protocol can be made even more efficient by forcing the next higher priority node to transmit immediately after receiving the packet from the highest priority node. Figure 3-12 is a transmission timing diagram detailing the operation of this hybrid MSAP protocol. The modem processing time between receiving and transmitting a packet is considered negligible when compared to the packet transmission and propagation delays. In order for the modified transmission scheme to work each packet must contain the source node number and a flag bit indicating whether or not more packets are to follow. This way a node may send multiple successive packets vice waiting for transmission priority. As seen at the bottom of Figure 3-12, the transmission scheme utilizes a greater portion of the channel for transmitting data than what could be achieved using TDMA. Upon receiving a packet a node will reset its minislot time boundary to the trailing edge of the packet (red dashed line.) When the priority node has no more packets to send it will defer transmission in the next minislot so that the next higher priority node can commence transmission. During the next minislot the node will listen to the channel to see if the next priority node has transmitted a packet. If no transmission is detected the receiving node will assume one of the following has occurred: 
1. If this is the first possible minislot the priority node can transmit, then the priority node does not exist in the cell (false clustering)

2. If this is any other minislot than the first, then the priority node does not have any packets to transmit (deferred transmission)

As previously discussed, a guard time will be inserted into the end of a frame to prevent the last transmission from interfering with adjacent nodes in the next cell hop. This means that a node cannot transmit a packet if the start time of the transmission is within $\mathrm{T}_{\mathrm{G}}+\mathrm{D}_{\mathrm{TP}}$ of the end of the hop frame time. 


\section{Chapter 4}

\section{Simulation}

This chapter details the steps taken in simulating the performance of the spatially dynamic cellular clustering algorithm for an ad-hoc network of nodes in the UACC. The simulations were performed using Matlab ${ }^{\circledR}$ scripts to define the distribution and placement of nodes, their mobility, the transmission losses in a shallow water environment, and the cell reuse pattern of the network. The overall performance of the distributed clustering algorithm is then characterized through simulation using the component algorithms presented in Chapter 3 and detailed in Appendix A.

\subsection{Performance Metrics}

\subsubsection{Receiver SIR Performance}

The metric used to characterize the ability of the acoustic modem to successfully receive and decode a transmission in the presence of interference is the receiver SIR. The receiver SIR in a distributed cellular network is strictly a function of the cell reuse pattern and the relative distance between nodes. SIR is characterized through simulation by summing the signal strength of each interfering 
node at the receiver and comparing it to the received signal strength of the communicating nodes in question. The simulated SIR assumes the MAC protocol allows only one node per cell to transmit at any given time.

\subsubsection{Networking Performance}

The two metrics used in measuring the networking performance of the distributed clustering algorithm are network connectivity and network stability. Network connectivity is defined as the percentage of network nodes that are fully connected during a complete hopping cycle. It is used to gauge the ability of the clustering algorithm to organize and maintain sufficient communication paths between nodes over time. The parameters in the spatially dynamic cellular clustering architecture that directly affect network connectivity are the number of hopping points, hopping distance, and node density.

Network stability is a measure of the distributed clustering algorithm's ability to preserve a stable network structure in the presence of node position errors. Node position errors are primarily generated from mobility and the age of the positional data maintained in the NODEPOSIT table. The network stability metric is used to determine how frequent a node must update its position to other nodes in the network.

\subsection{Node Placement and Mobility Model}

A key component in the simulation of the spatially dynamic cellular clustering algorithm is a representative node model that emulates the relative placement and mobility of sensor platforms within an UAN. The model assumes the only forces acting on a node are due from thrusters and not from any mean fluid flow. 

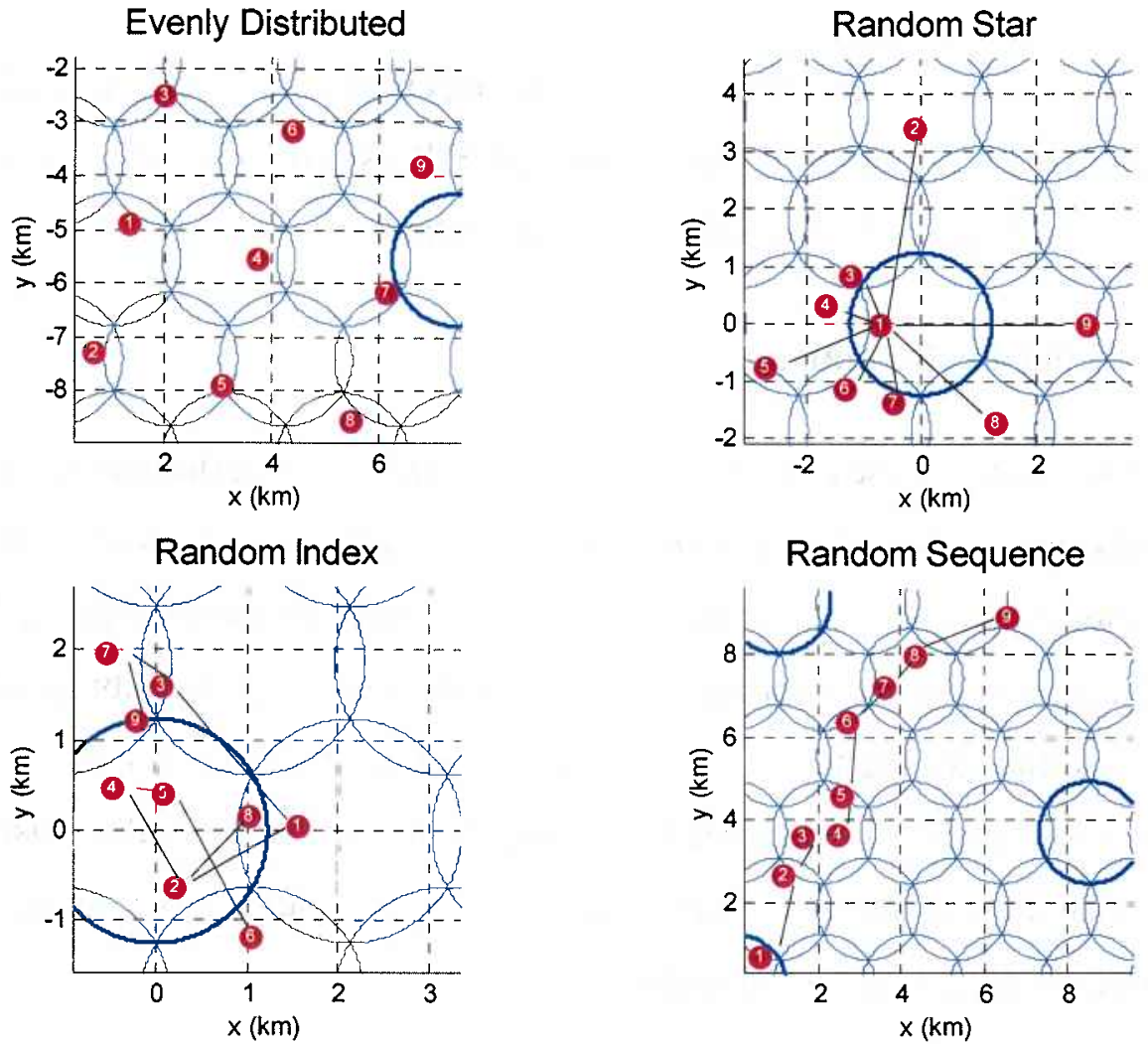

Figure 4-1 Node distributions used in the simulation of the spatially dynamic cellular clustering algorithm. The evenly distributed placement of nodes is useful for simulating a specific node density. The node placement in the random star distribution closely mimics the dispersion of several mobile nodes deployed from a specific point. The random index distribution is a general pupose distribution that is used to define the starting node placements for a random walk simulation. The nearly straight-line appearance of the random sequence distribution is used to simulate the placement of air dropped nodes.

The node model used in simulation was developed with two UAN application scenarios in mind:

1. Coordinated operations between multiple AUVs tasked to conduct a moving search of a littoral region

2. Ad-hoc deployment of stationary sensor platforms and information gateway(s) interspersed with AUVs

The four different node distributions used in the simulations can be seen in Figure 4-1. The evenly distributed distribution arranges nodes into an evenly spaced grid. 
Node Placement and Mobility Model

The angle of the node grid relative to the coordinate axes is randomly chosen from a uniform distribution of $(0,2 \pi)$. This node distribution is particularly useful in simulating the performance of the distributed clustering algorithm for a given node density. The random star distribution places nodes in a radial pattern around a randomly chosen node. This distribution is particularly well suited to simulate the dispersion of mobile nodes that have been deployed from a single point. The angular separation between each radial node position is set to $2 \pi /(\mathrm{N}-1)$ and the radial displacement is randomly chosen from a uniform distribution of $(0, L)$, where $L$ is the maximum displacement allowed between nodes. In the random index distribution, the relative position of node $n$ is based on the position of a randomly chosen node from the set $\{0 \ldots n-1\}$. This is a general purpose node distribution that is used to define the starting position of nodes in a random walk simulation. Using Figure 4-1 as an example, the relative angle and displacement of node 8 is based on the position of node 2. The random star and random index distributions are primarily used to simulate an ad-hoc deployment of nodes in an UAN. The random sequence distribution is similar to the random index distribution, except the relative position of node $n$ is based on the position of node $n-1$. This distribution is used to simulate the sequential deployment of nodes along a coastline, similar to that found in airdropped or rapidly-deployed units.

The movement of nodes within the UAN is modeled as a random walk. The uniform random variables $\Theta$ and $\Gamma$ are used to define the relative direction and distance of each step in the walk as shown in Figure 4-2. Applying these random parameters at each node, the relative motion between a set of both stationary and mobile nodes in a static field flow can be generated and used in characterizing the performance of the spatially dynamic cellular clustering scheme in the presence of node mobility. The maximum distance between steps in the random walk is based on the maximum speed of the node $\left(s_{\max }\right)$ and each time step $\left(t_{\text {step }}\right)$ in the simulation. 


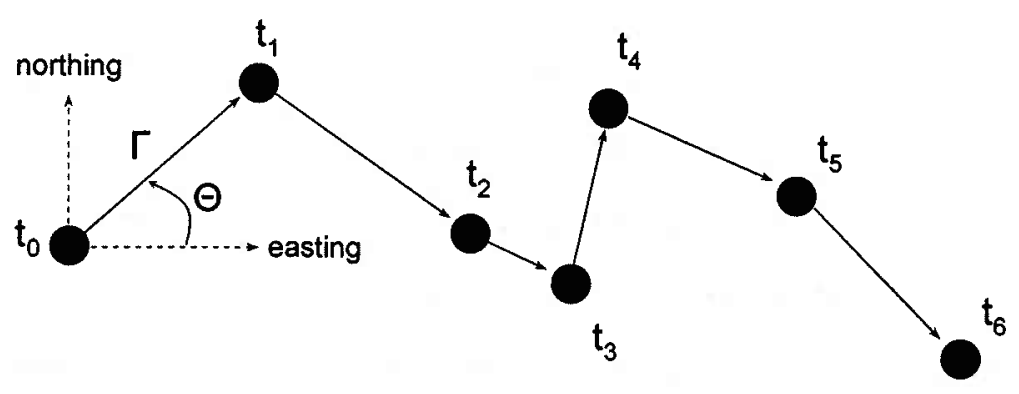

Figure 4-2 Example of a random walk using the parameters $\Theta$ and $\Gamma$ to define the next northing and easting position of a node.

The northing and easting position of the node at each point in the walk is calculated by

$$
\begin{gathered}
\Gamma=\text { uniform }\left(0, s_{\max } t_{\text {step }}\right) \\
\Theta=\text { uniform }(0,2 \pi) \\
\text { easting }(t)=\text { easting }\left(t-t_{\text {step }}\right)+\Gamma \cos (\Theta) \\
\text { northing }(t)=\text { northing }\left(t-t_{\text {step }}\right)+\Gamma \cos (\Theta)
\end{gathered}
$$

\subsection{Receiver SIR Simulations}

\subsubsection{Simulation Setup}

The simulation of receiver SIR examines $K=7,12$, and 19 cell reuse patterns generated using Dikstra's minimum distance algorithm. These cell patterns are then used to segment the geographic area covered by the UAN under simulation as show in Figure 2-11. The radius of the cells, corresponding to the PRD90 of the modem, is set to the $10 \mathrm{~dB}$ empirical absorption limit for seawater using a $25 \mathrm{kHz}$ mean center frequency for the acoustic modem.

$$
\text { cell_radius }=\frac{10}{\alpha}(\mathrm{km})
$$


The water depth of the UACC is set to 100 meters with the incident angle of the empirically dominant eigenray between the source and the receiver set to 45 degrees. The transmit power of the acoustic modem is assumed to be fixed at 1 watt. The actual power level chosen for simulation is not important as the receiver SIR is strictly a function of range.

\subsubsection{Worst Case Receiver SIR}

The simulation of worst case receiver SIR assumes the worst case positioning of transmitter, receiver, co-channel interferer, and adjacent channel interferer nodes within the cells and cell clusters. Figure 4-3 illustrates the worst-case positions of these nodes ( 48 nodes in all) in a $\mathrm{K}=7$ cell reuse pattern. All adjacent channel interferer cells within the ring formed by the outer edge of the co-channel cells are included in the simulation. The worst-case position of each interfering node is
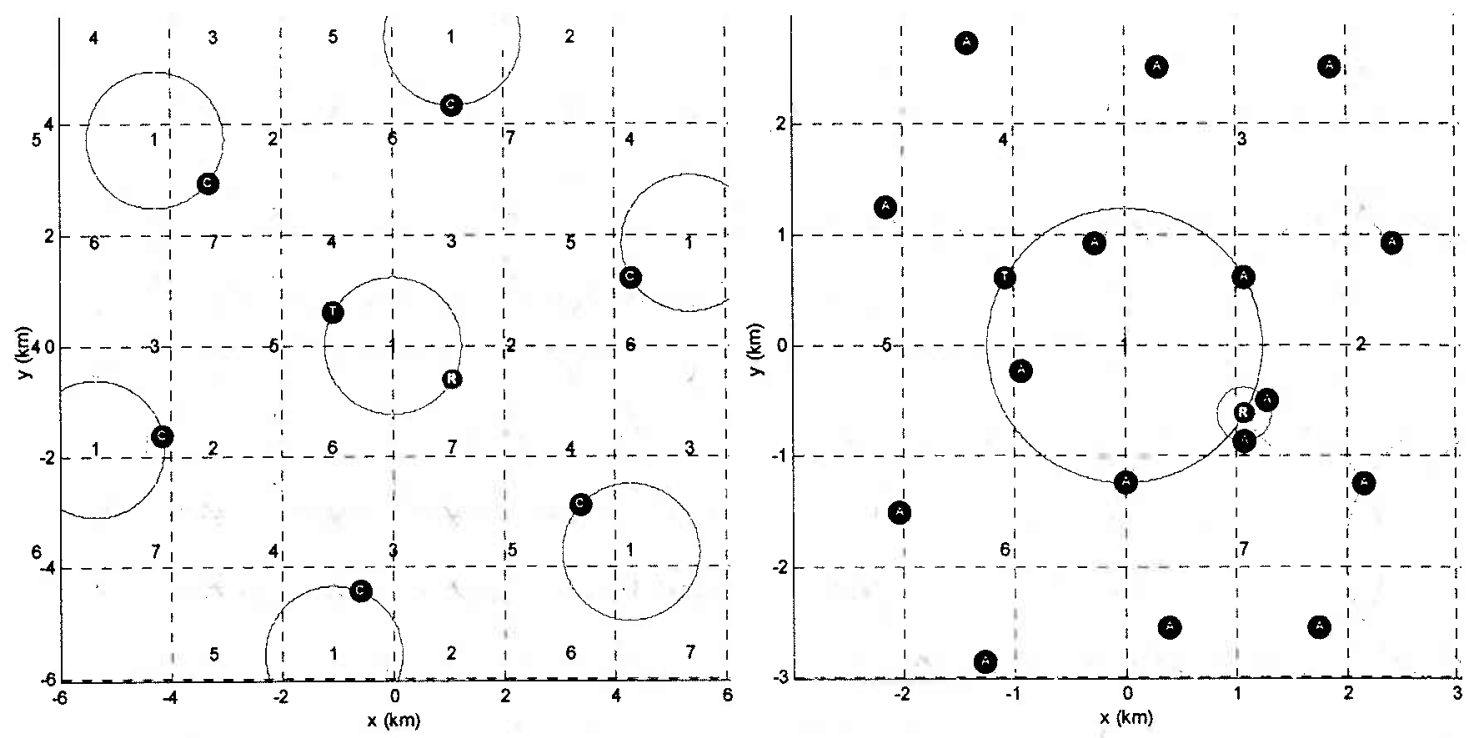

Figure 4-3 Worst-case positioning of transmitter (red), receiver (green), co-channel interferer (blue), and adjacent channel interferer (black) in $\mathrm{a} K=7$ cell reuse pattern. The figure on the left shows the relative position of the co-channel interferers while the one on the right shows the adjacent channel interfererd. The red ring represents the proximity limit between the receiver and nearby adjacent channel nodes 


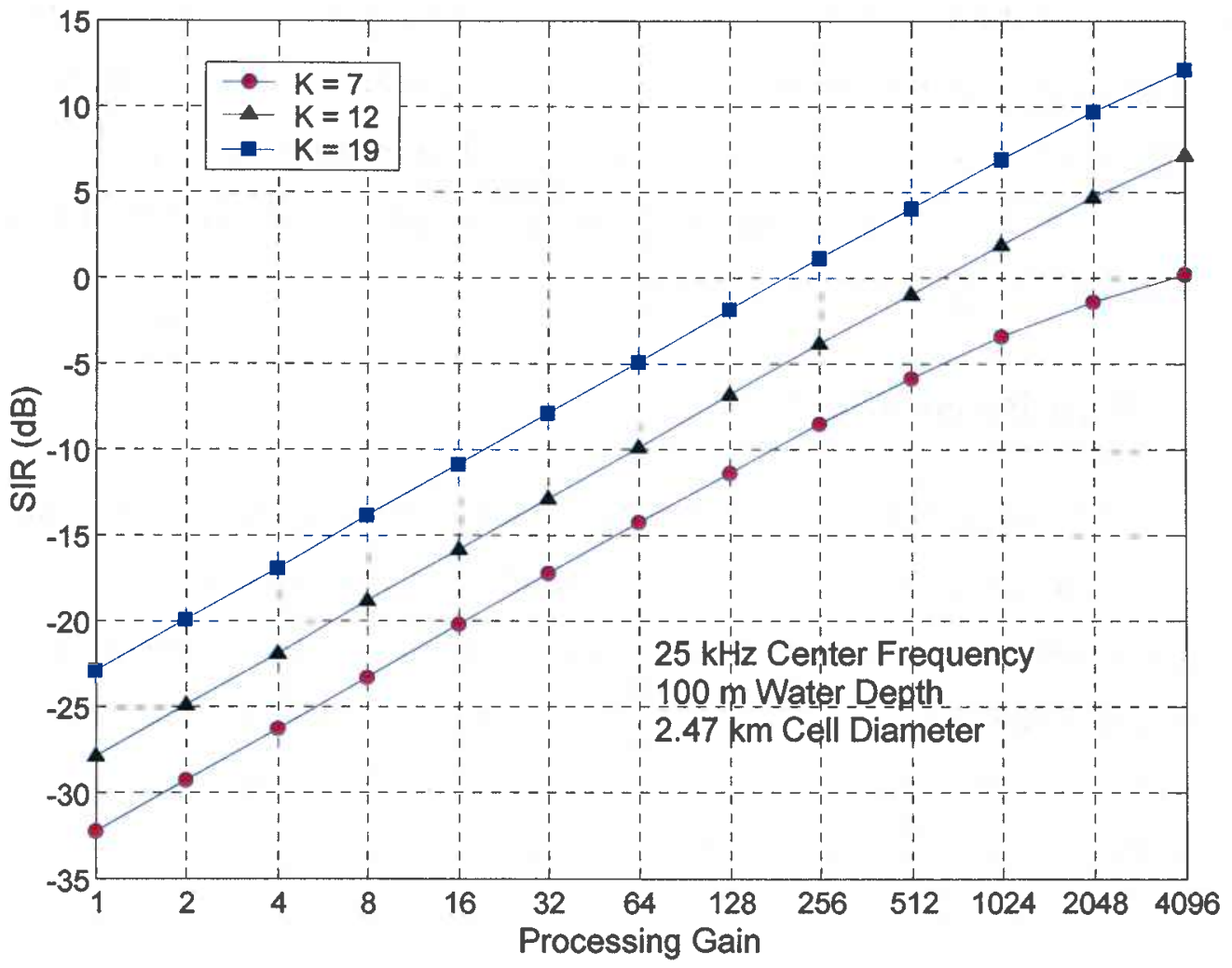

Figure 4-4 Worst case SIR versus processing gain for $K=7,12$, and 19 cell reuse patterns

calculated by offsetting the center point of each interferer cell by a distance equal to a cell radius in the direction of the receiver under inspection. In addition, the offset of those adjacent channel nodes that could be potentially pre-clustered by the distributed clustering algorithm were reduced by the proximity limit (red ring in Figure 4-3) to ensure their contribution to the worst-case interference. Through examination of Figure 4-3 the largest component of interference is expected to come from those nodes within close proximity to the receiver. The transmitter and receiver were placed at opposite ends of the center cell and positioned such that the average distance to any node in the cell reuse pattern is minimized producing the worst-case signal strength from each interfering node. The signal strength received from each interferer node was then calculated using equation (3.3). Finally equation (3.4) was used to calculate the SIR at the receiver. A plot of the worst-case SIR 
versus processing gain for $K=7,12$ and 19 cell reuse patterns can be seen in Figure 4-4. Examination of the results shows that a modest gain in SIR can be achieved for a successively larger cell reuse pattern. A larger cell reuse pattern increases the distance between co-channel cells and increases the receiver SIR.

\subsubsection{Average Receiver SIR}

In order to simulate the average receiver SIR in an ad-hoc UAN the transmitter, receiver, and interferer nodes in Figure 4-3 were randomly placed within their assigned cells. Nodes that are within the proximity limit of the receiver were dropped from the SIR calculation to reflect having been clustered into the same cell as the receiver. Using these randomly distributed node positions the simulation setup above was reiterated 1000 times for $\mathrm{K}=7,12$, and 19 to statistically characterize the average receiver SIR. The processing gain for this particular simulation is set to 1 ( 0

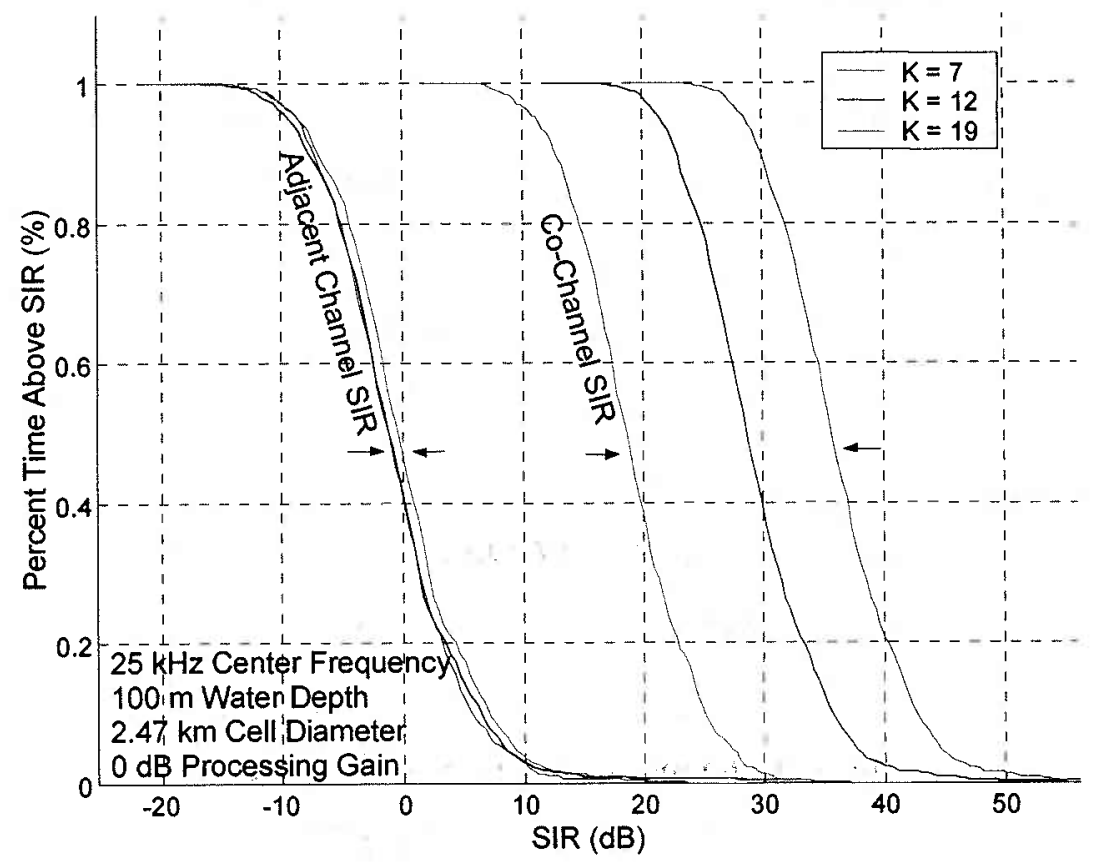

Figure 4-5 A comparison between the average time above SIR for co-channel and adjacent channel interferers. Adjacent channel users are the predominant source of interference at the receiver 


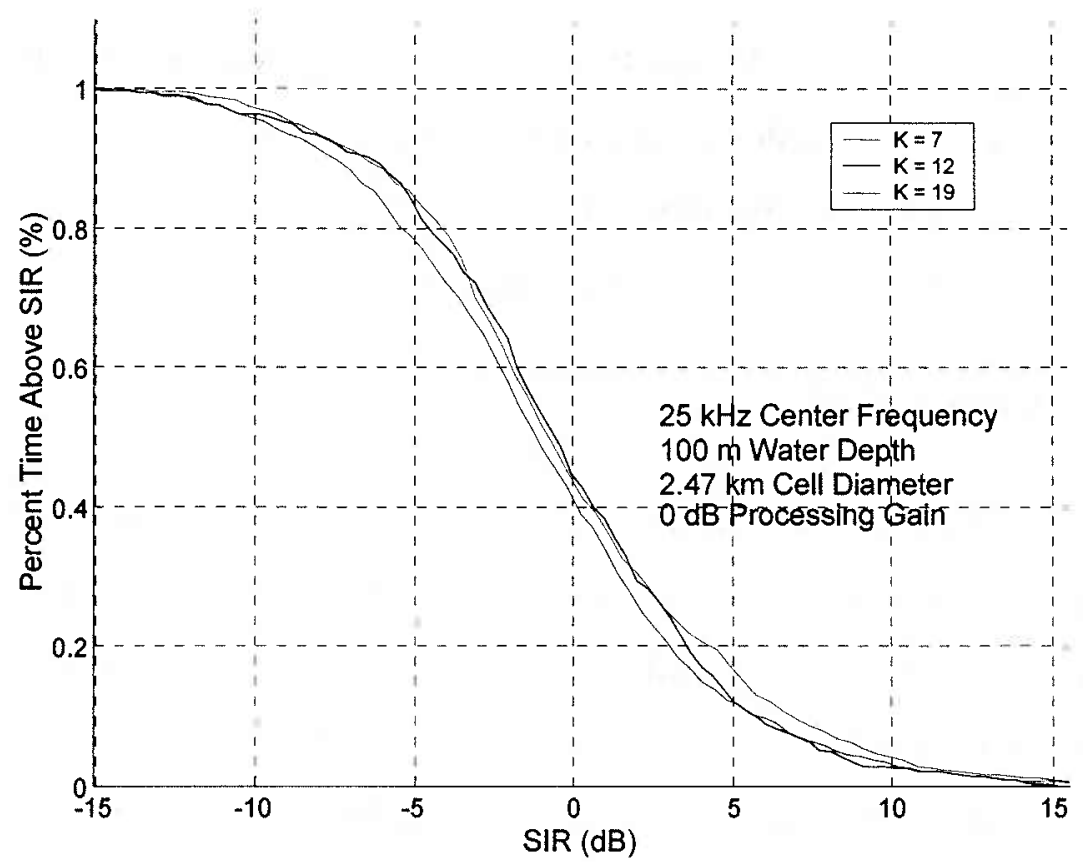

Figure 4-6 Average time above SIR for $K=7,12$, and 19 cell reuse patterns. The close spcaing of the SIR curves stems from the fact that the dominant component of the interference at the receiver is generated by adjacent channel users and is independent of the cell reuse pattern

$\mathrm{dB})$ to emphasize the relative magnitudes of co-channel and adjacent channel interference. A comparison between the average time above SIR for co-channel and adjacent channel interferers can be seen in Figure 4-4. The results show that the dominant component of the total interference at the receiver is generated by adjacent channel interferers. Consequently, the receiver SIR more closely follows the adjacent channel SIR which is independent of the cell reuse pattern. This implies that in order to substantially increase the available SIR at the receiver a higher processing gain is required. The average time above a given $\mathrm{SIR}$ for $\mathrm{K}=7,12$, and 19 cell reuse patterns can be seen in Figure 4-6. The close spacing of the SIR curves steams from the fact that adjacent channel user generate the predominant component of the interference at the receiver. As such, the receiver SIR more closely follows the adjacent channel SIR. The effect of various processing gains on the average receiver SIR can be estimated by shifting the entire curve to the right by the amount 
Receiver SIR Simulations
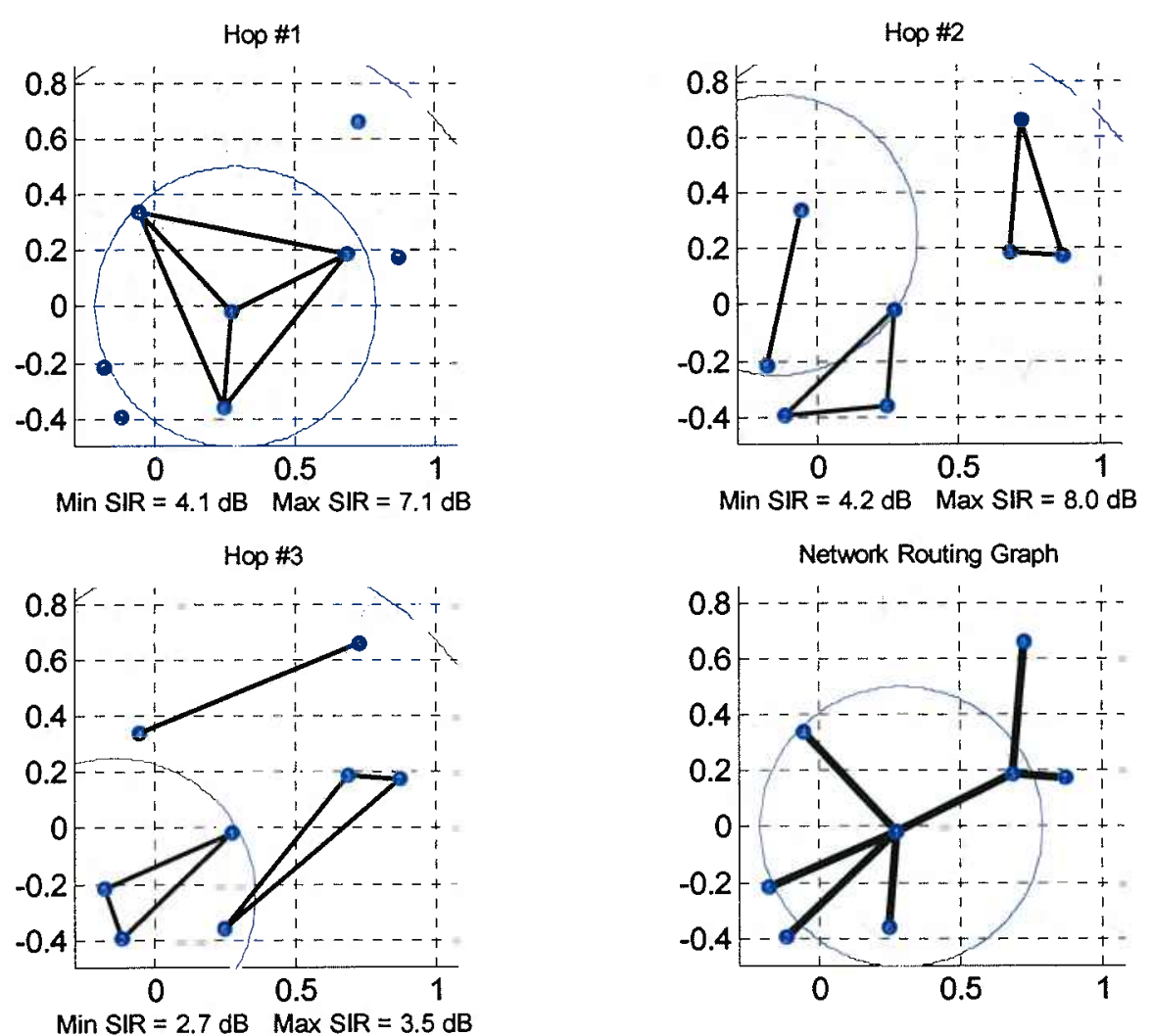

Figure 4-7 Receiver SIR example for an UAN cosisting of 8 nodes.

of the processing gain. For example, if the processing gain is $8(9 \mathrm{~dB})$ then the SIR at the receiver will be above $9 \mathrm{~dB}$ for approximately $50 \%$ of the time.

The simulation results presented thus far seek to characterize statistically the worst case and average SIR performance of a receiver employed in a distributed cellular network. While the above examples considers all nodes within the area bounded by the first tier co-channel cells for SIR calculations, the example shown in Figure 4-7 is more representative of a typical UAN deployment scenario using 8 stationary nodes. The nodes are first clustered into cells by the spatially dynamic cellular clustering algorithm, and then analyzed for the minimum and maximum SIR at each hop point. The spread spectrum modem simulated in the example has a processing gain of $7(8.4 \mathrm{~dB})$ with a $25 \mathrm{kHz}$ mean center frequency and a PRD90 of 
$1 \mathrm{~km}$. The minimum and maximum SIR for any pair of nodes at each hop point is determined by systematically comparing the signal strength between a pair of nodes co-located in a cell to the interference generated by nodes simultaneously transmitting in the adjacent cells. The calculated dynamic range of SIR for any node in the example network is between 2.7 and $8.0 \mathrm{~dB}$ at any given time.

\subsection{Networking Performance Simulations}

The simulation of network performance differs slightly from receiver SIR in that the spatially dynamic cellular clustering algorithm is only concerned with the relative positioning of nodes within cells and not the range and frequency dependent transmission loss between node positions. In this way, the relative position of nodes can be normalized by the cell diameter to produce a non-dimensional representation of the network structure. Using this notion, the networking performance of the clustering algorithm for a given set of network parameters (e.g., hopping points, hopping distance, etc.) can be extended to any other set of network parameters without a loss in generality.

The network simulations presented in this section are separated into two series. The first series of simulations intend to characterize the networking performance of the spatially dynamic cellular clustering algorithm for variations in cellular geometry parameters, node density, and node mobility. These results can then be used by the UAN designer to determine the network parameters that optimize network connectivity for a given application. The second series of simulations will demonstrate the ability of the distributed clustering algorithm to maintain a stable network structure in the presence of positional errors. This information can then be used to determine the maximum time between node position updates that can ensure a stable network structure. 
Networking Performance Simulations

\subsubsection{Simulation Setup}

The simulation setup in this section uses the same node placement and mobility model described in Section 4.2. First, an ad-hoc set of 36 nodes is generated using one of the node distributions in section 4.2.1. The NODEPOSIT table for each node is completed using only the nearest neighbor positional information of those nodes within PRD90 of each other. The network parameter under examination (e.g., hopping points, mobility, etc.) is then defined and simulated for each node in the network using the spatially dynamic cellular clustering algorithm. At the completion of each simulation the nodecell_map from each node is compared to verify they convergence to the same network structure. Only those nodes that are within the PRD90 of one another are considered in the comparison since it is assumed that any pair of nodes separated by a distance greater than the PRD90 cannot communicate. The nodecell_map and position information of each node in the network is then used to construct a minimum spanning tree between nodes. The network connectivity is calculated by comparing the number of nodes included within the largest minimum spanning tree to the total number of nodes in the network. Finally, the above process is repeated 1000 times for each of the 4 node distributions and statistically averaged to characterize the effects of the network parameter on network connectivity. 


\subsubsection{Cell Hopping Parameters and Network Connectivity}

The spatially dynamic cellular clustering architecture requires three key parameters to control the behavior of the hopping scheme: the number of hopping points (hop_points), the distance between hop points (hop_distance), and the time spent at each hop point (hop_period). The hop_points and hop_distance parameters directly affect the clustering performance of the distributed clustering algorithm by defining the physical geometry of the hopping pattern. It is the dynamics of the cell geometry that is instrumental in the clustering nodes and allocation of communication resources. On the other hand, the hop_period is set by
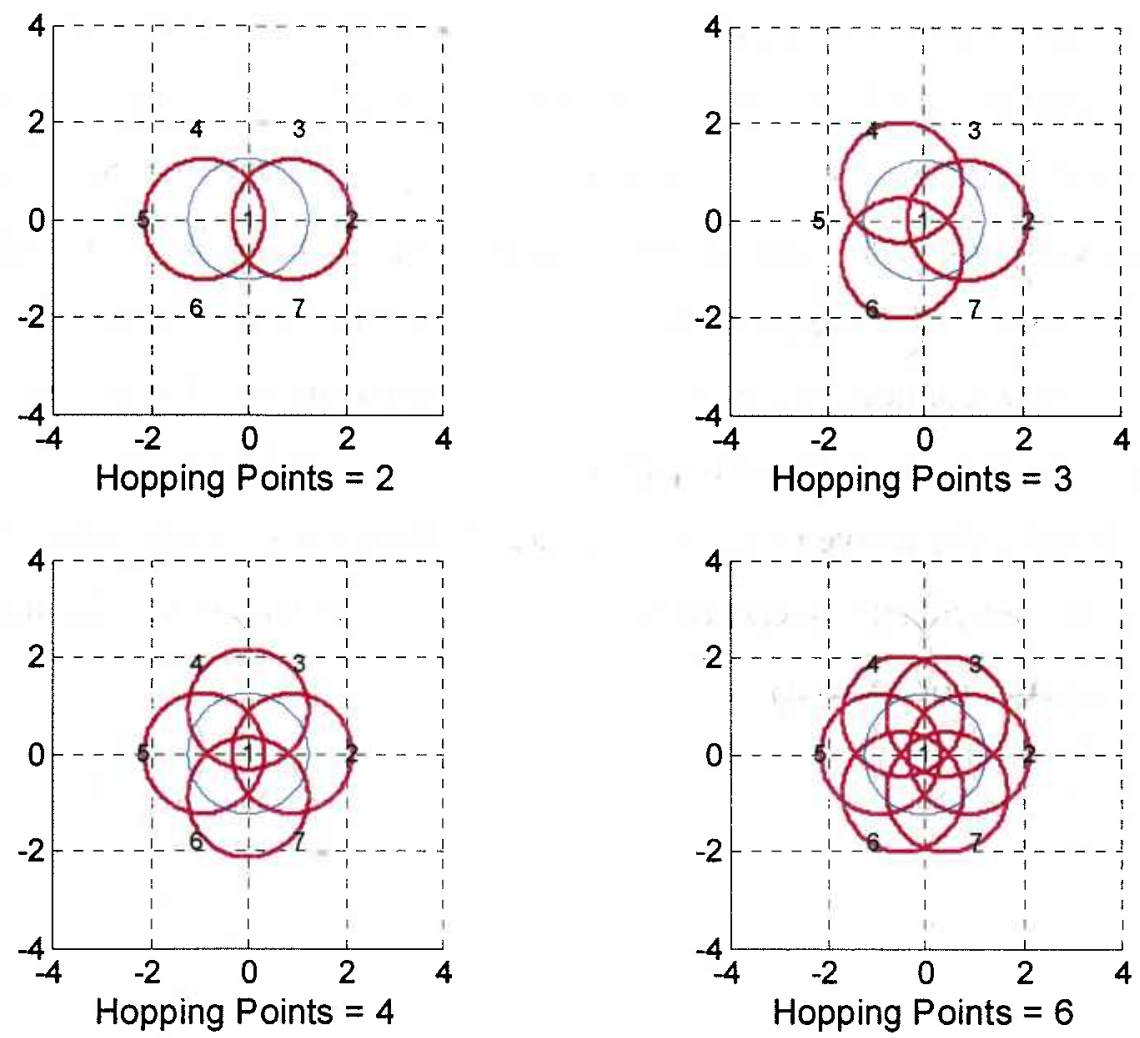

Figure 4-8 Diagram of hopping points (hop_points) used in simulation. The hopping distance (hop_distance) using the diagram was set to $75 \%$ of the cell radius (cell_radius). The blue cell is centered on the network reference coordinate with each red circle representing the relative shift, or "hop", in the layout of the cell pattern. 
Networking Performance Simulations
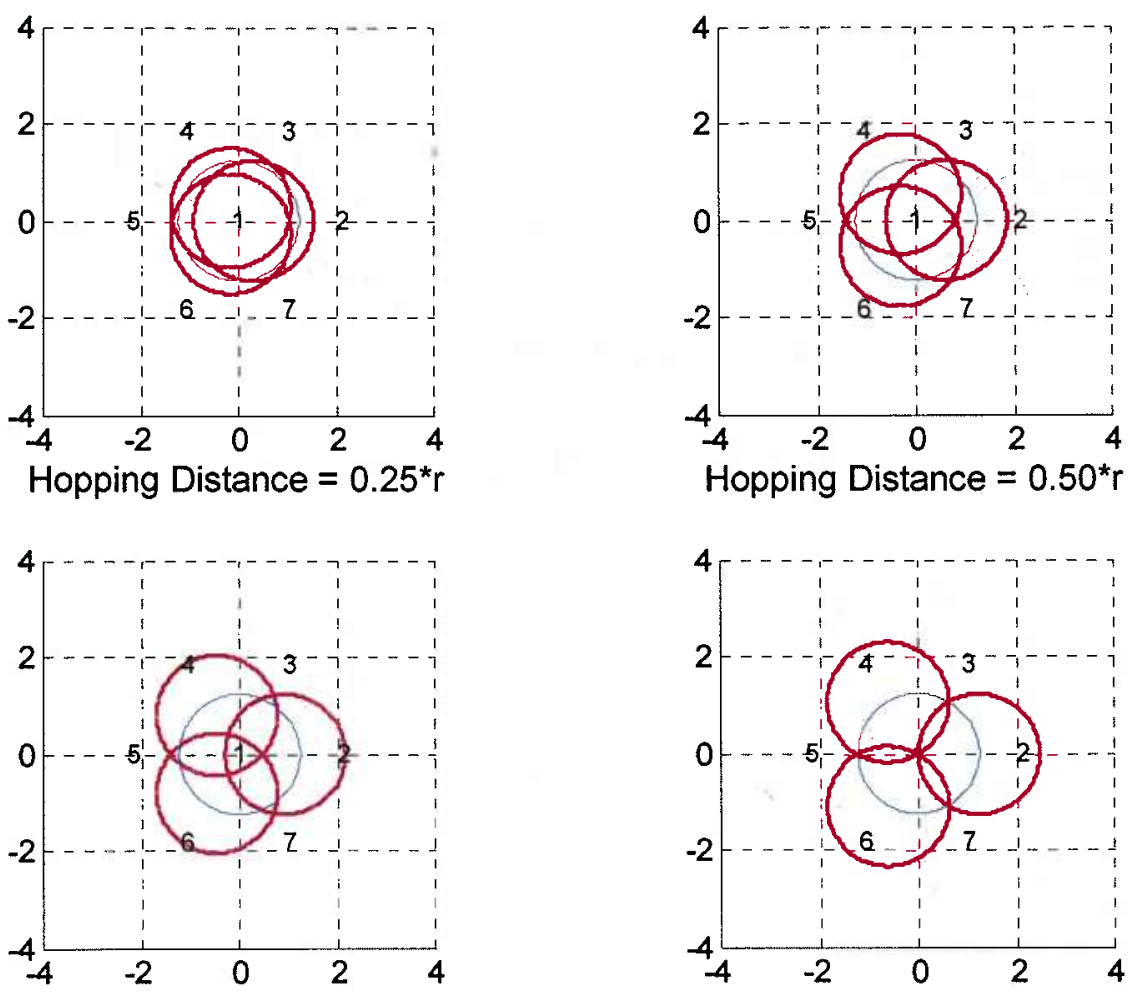

Hopping Distance $=0.75^{\star} \mathrm{r}$

Hopping Distance $=1.00^{*} r$

Figure 4-9 Diagram of hopping distance (hop_distance) for a 3 hopping point pattern. The blue cell is centered on the network reference coordinate with each red circle representing the relative shift, or "hop", in the layout of the cell pattern.

the MAC protocol to provide a sufficient period of time for clustered nodes to communicate at each hop point and does not directly affect clustering performance.

The clustering performance of the distributed clustering algorithm is simulated using hopping points of $2,3,4$, and 6 in conjunction with hopping distances that are $25 \%, 50 \%, 75 \%$ and $100 \%$ of the cell radius (cell_radius). The diagram in Figure 4-8 shows the relative geometry and position of the network reference cell at each hop point with the hop_distance set to $75 \%$ of cell_radius. The diagram in Figure 4-9 illustrates the relative offset between each hop point in a 3 hop pattern. The density of nodes in the UAN is simulated by varying the relative distance between nodes situated in an equally spaced grid of 36 nodes. Without a 


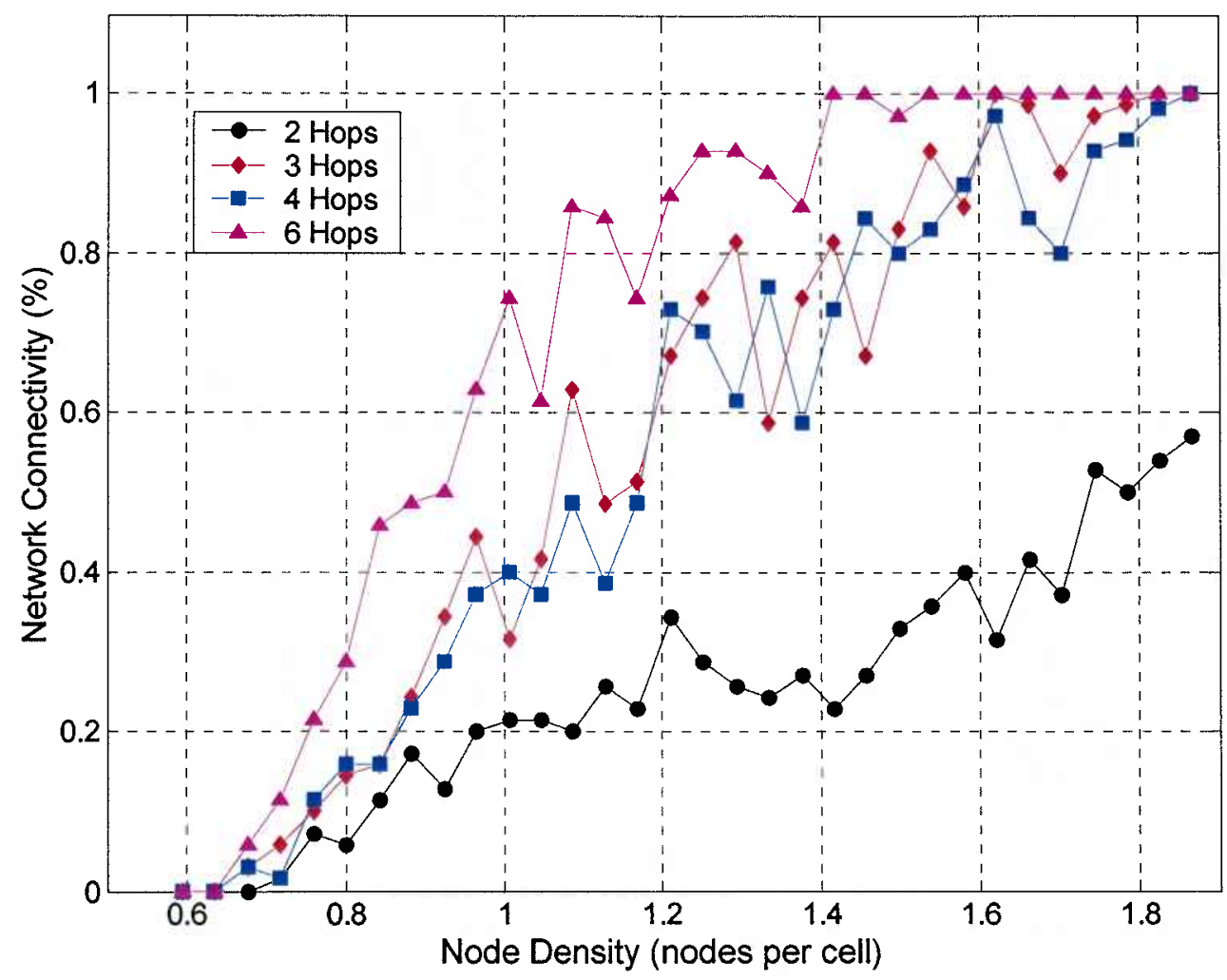

Figure 4-10 Network connectivity versus node density for hopping points of 2, 3, 4, and 6 . The hopping distance is set to $75 \%$ of the cell radius.

loss in generality, the simulated node density is then normalized by the cell coverage area to produce a node density that is measured in nodes per cell.

In the first simulation, the clustering performance for hopping points of 2, 3, 4 , and 6 and a hopping distance of $75 \%$ of cell_radius are examined against varying degrees of node density. The simulated results of network connectivity versus node density can be seen in Figure 4-10. The results show that as the number of hopping points increases the percentage of fully connected network nodes in a hopping cycle also increases. This outcome is expected since the available clustering area of each cell is expanded by the combination of multiple hopping points and the hopping distance. The overall effect produces a higher diversity of node clusters within a given set of nodes and a subsequently higher level of network connectivity. 


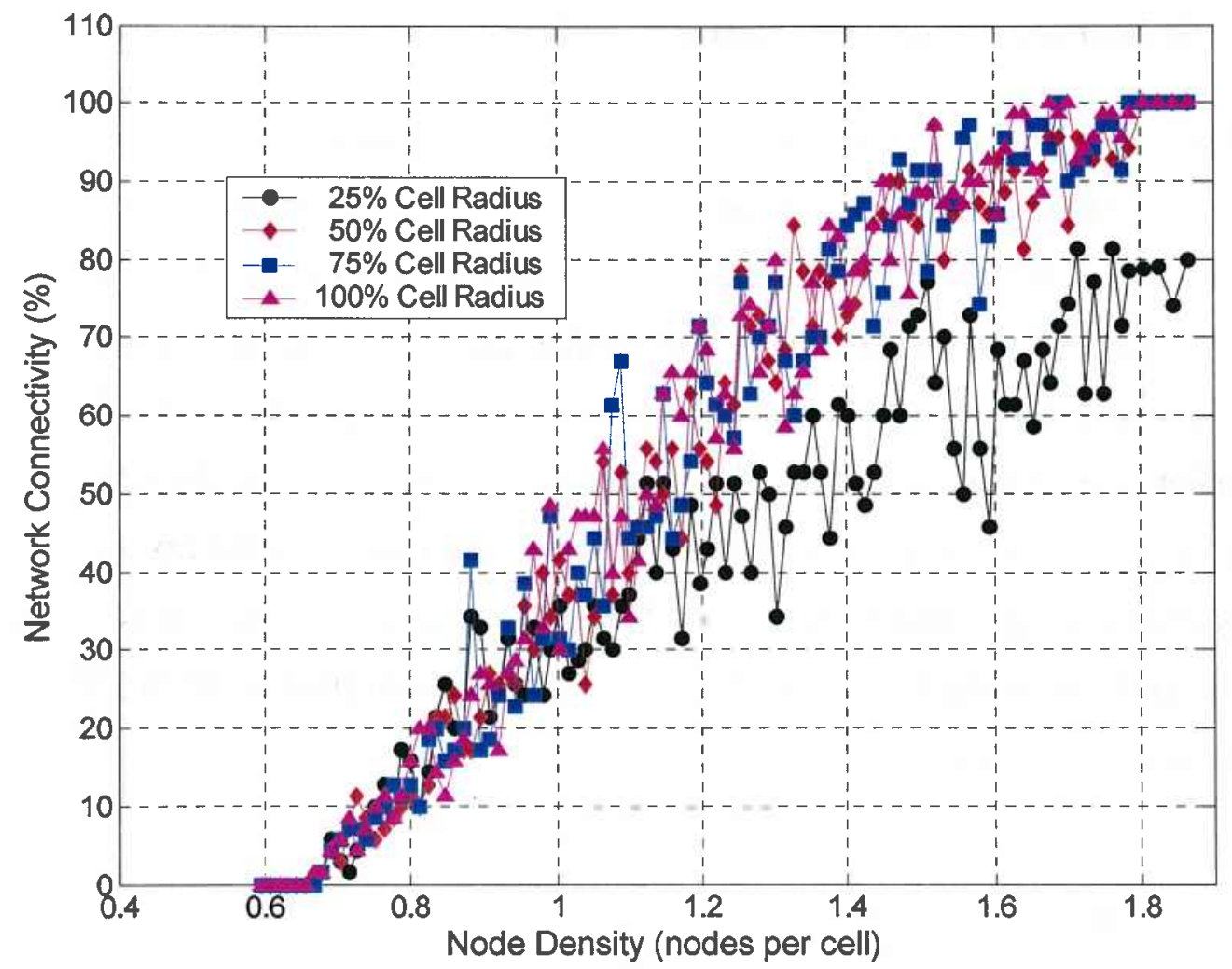

Figure 4-11 Network connectivity versus node density for hopping distances equal to $25 \%$, $50 \%, 75 \%$, and $100 \%$ of the cell radius and a hopping point pattern of 3 .

In the second simulation, the networking performance for hopping distances equal to $25 \%, 50 \%, 75 \%$ and $100 \%$ of cell_radius are examined for a hopping point pattern of 3. A plot of the simulated results for network connectivity versus node density can be seen in Figure 4-11. The results show that as the hopping distance increases the percentage of fully connected network nodes in a hopping cycle also increases. Interestingly, there is no additional gain in network connectivity for hopping distances beyond $50 \%$ of cell_radius. This stems from the fact that the effective coverage area of each cell from hopping completely overlap at a hopping distance equal to $50 \%$ of cell_radius. Any additional lengthening in the hopping distance merely shifts the position of the overlapping regions producing the same network connectivity for the given number of hopping points. 


\subsubsection{Node Position Errors and Network Stability}

One of the more desirable characteristics of the spatially dynamic cellular clustering algorithm is the ability of each node executing the algorithm to converge to the same network structure using only the positional information of its nearest neighbor nodes. This supposition assumes that the time-dependent positional information generated by each node is an accurate fix of its position in the UAN. The combination of node mobility, the accuracy of navigational equipment, and communication delays in reporting a fix to other nodes introduce uncertainty into the accuracy of a node's position in the UAN. Over time these inaccuracies can result in clustering conflicts by the distributed clustering algorithm that produce instability in the network structure derived at each node.

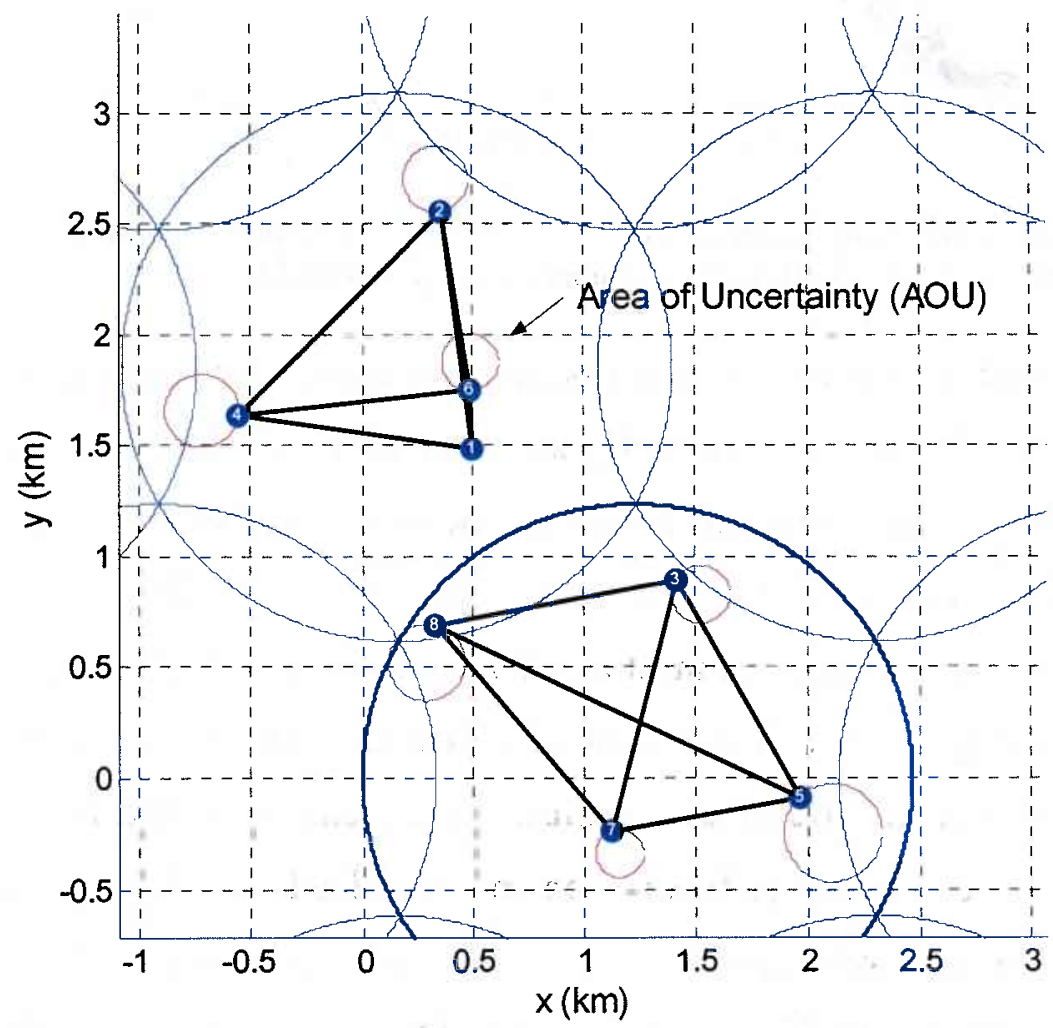

Figure 4-12 Uncertainty in the position of a node. The red ring represents the area of uncertainty (AOU) generated by node 1 for each of its nearest neigbor nodes. The size of the AOU is based on the maximum speed of the node, the age of the last fix, and the accuracy of the navigation equipment. 
Networking Performance Simulations

The node positional data in the NODEPOSIT table represents where a nearest neighbor node was located at a particular time. Using the last known fix and maximum speed of the node, the age of the fix, and the accuracy of the navigation equipment the spatially dynamic cellular clustering algorithm generates an area of uncertainty (AOU) that bounds the current position of the node in the UAN. Since the exact speed and direction of a node since its last reported fix is not known the worst-case position of the node is now assumed to be somewhere within this AOU. The example in Figure 4-12 illustrates the relative size and position of the AOUs generated by node 1 for its nearest neighbor nodes. The distributed clustering algorithm must now use these AOUs to generate a stable network structure. If the AOU grows too large, then the network structures generated at each node will no longer converge to a single, stable network structure. The goal of this simulation is to determine at what point does the size of the AOU (amount of positional error) impact the stability of the clustering algorithm.

The metric used to determine the stability of the network structure is the average number of link changes for a given amount of positional error. The positional error is used to define the maximum diameter of the AOU. Without a loss in generality, it is simulated as a non-dimensional quantity that is a fractional amount of the cell radius. The four node distributions presented in section 4.2 were used in the simulation for comparative analysis. First, an ad-hoc set of 36 nodes was generated using one of the four node distributions. The distributed clustering algorithm was then executed at each node to produce a nodecell_map using the error-free node positions. Next, the position of each node was offset by a given amount of positional error to represent the maximum diameter of each AOU. The distributed clustering algorithm was again executed at each node to produce a nodecell_map using the simulated AOUs. Next, the nodecell_map from both executions were compared to determine how many nodes were clustered into different cells for a given amount of positional error. Finally, the number of link 


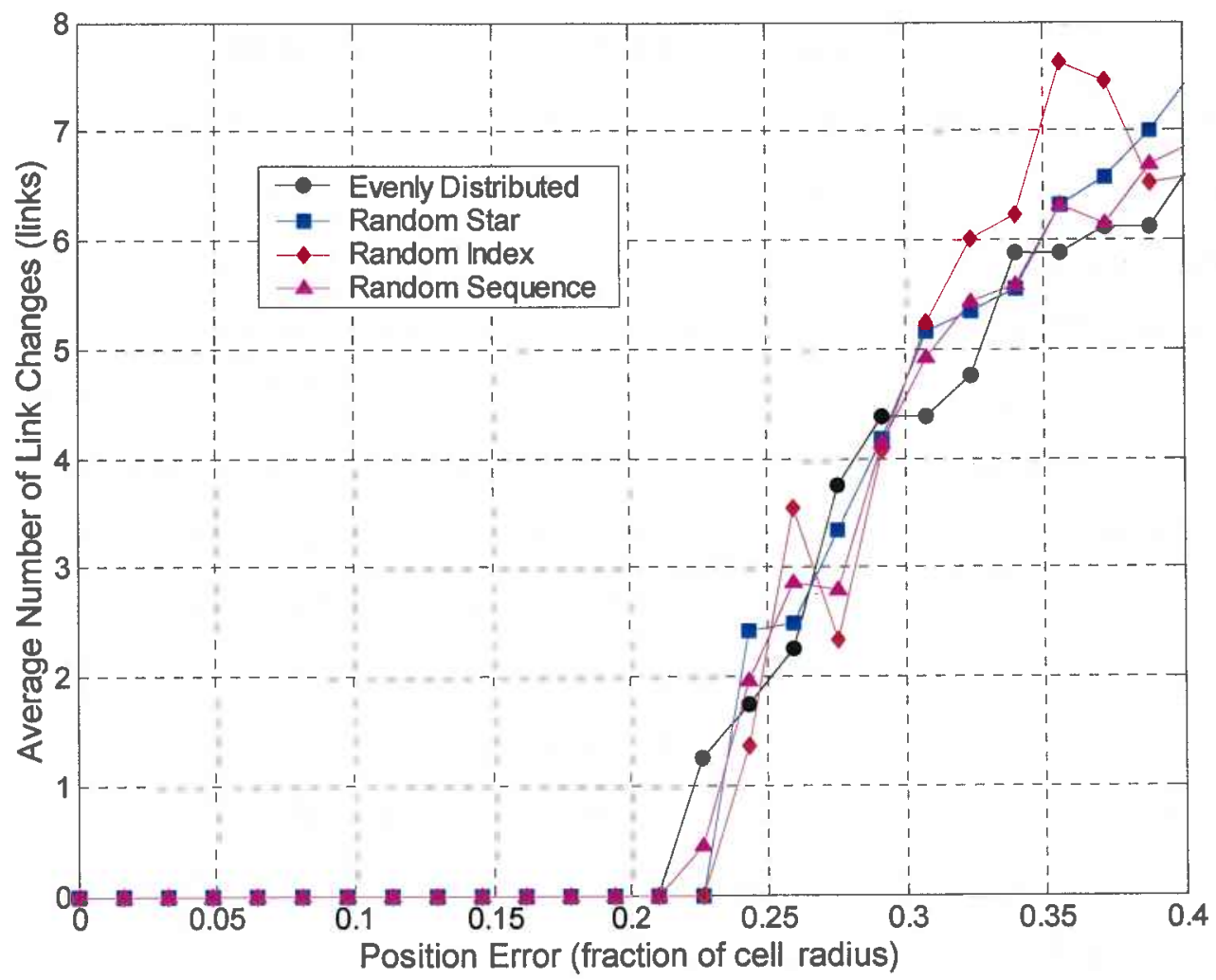

Figure 4-13 Position error versus average number of link changes for the 4 different node distributions using 36 nodes. The results show that if the maximum error in a node's position does not exceed $21 \%$ of the cell radius then a stable network structure can be maintained by the distributed clustering architecture.

changes for the network was determined using those nodes that had changed their cell membership due to the introduction of positional errors. The above process was repeated 1000 times for each of the four node distributions to provide a statistical average of the results. A plot of the average number of link changes versus position error for each of the four simulated node distributions can be seen in Figure 4-13. The results show that for position errors less than 0.21 of the cell radius the spatially dynamic cellular clustering algorithm will converge to a stable network structure at each node executing the algorithm. As an example, if the diameter of each cell in the network was set to the $10 \mathrm{~dB}$ empirical absorption limit for an acoustic modem with a center frequency of $25 \mathrm{kHz}$, then the maximum amount of position error $\left(\varepsilon_{\text {posit }}\right)$ that 
will not introduce instability in the network structure is given by the empirical relation

$$
\varepsilon_{\text {posit }}=0.21\left(\frac{1}{2}\right)\left(\frac{10 d B}{\alpha(25 k H z)}\right) \cong 260 \text { meters }
$$

This information can then be used to determine how frequent a node must report its updated position to its nearest neighbors. Assuming a maximum node speed $\left(s_{\max }\right)$ of 3 meters per second, and a 10 meter error in the accuracy of the navigation equipment $\left(\varepsilon_{n a v}\right)$, the maximum period of time between position updates $\left(T_{\text {update }}\right)$ is given by

$$
T_{\text {update }}=\frac{\varepsilon_{\text {posit }}-\varepsilon_{\text {nav }}}{s_{\max }}=\frac{260 \mathrm{~m}-10 \mathrm{~m}}{3 \mathrm{~m} / \mathrm{s}} \cong 83 \text { seconds }
$$

In this example, each node must report its updated position to its nearest neighbor nodes every 83 seconds to preclude the introduction of instability in the network structure. Using this information, the maximum length of the hopping period and the position update mechanism imbedded in the MAC protocol must respect this time limit to ensure network stability. 


\section{Chapter 5}

\section{Conclusions}

The primary objective of this thesis was the development and validation of a distributed networking protocol suitable for ad-hoc deployments of both stationary and mobile nodes operating in a shallow water environment. The spatially dynamic cellular clustering scheme was based upon proven networking concepts extrapolated from classical cellular communications and wireless network theory and then conformed to the modeled UACC. Modern spread spectrum acoustic modem technology was incorporated into the network design to provide enhanced interference immunity, multi-user capability, and lower probability of intercept for UANs. The overall network design addressed the problem of communication resource allocation, communication channel reuse, and the integration of a MAC protocol for use by exclusive subsets of nodes in an undersea environment. The various environmental, operational, and design parameters that directly affected the networking performance, acoustic link stability, and modem receiver signal integrity of the spatially dynamic cellular clustering scheme were analyzed through simulation to provide the network designer with the necessary information to implement the design in an UAN. 
Contributions of the Thesis

\subsection{Contributions of the Thesis}

In an effort to make the spatially dynamic cellular clustering scheme applicable, the thesis work focused on the cellular implementation, design choices, and analysis of the key parameters deemed relevant to its operation in an UAN. Some of the major contributions of the work include:

- A distributed networking architecture that reduce the amount of network management overhead by geographically allocating communication resources based on the relative position of nodes in a cellular framework.

- Characterization of receiver SIR performance in a distributed cellular network for various cell reuse patterns, cell sizes, and processing gains. This information is useful in determining the minimum requirements of an acoustic modem for an UAN.

- A distributed clustering scheme that dynamically repositioned the cellular framework in time to provide diversity in the available communication links between adjacent nodes in the network. Through simulation, it has been shown that the hopping scheme can provide a relatively high level of connectivity between nodes in the network.

- A distributed clustering algorithm that dynamically reconfigures the structure of the UAN using only the position information of nearest neighbor nodes and not the position of every node in the network. The algorithm demonstrated its ability to converge on a stable network structure independent of the node executing the algorithm and in the presence of moderate uncertainty in node positions. A link stability metric was derived from simulation that provided UAN designers with a key parameter needed in determining the position update rate of nodes in the network. 


\subsection{Future Work}

There are three aspects of the spatially dynamic cellular clustering scheme that are amiable to future development. First, the application of the cellular framework is specific to a relatively shallow water environment since the cell geometry is only represented in two dimensions (2D). The cellular framework can be extended to deep water applications by generating three dimensional (3D) cell reuse patterns. While the receiver SIR performance should be similar in 3D, the cellular hopping pattern will have to assume a 3D pattern as well to achieve a similar level of network connectivity from the clustering algorithm. Second, the MAC protocol presented in the design seeks to highlight the performance advantages afforded by sharing communication resources with smaller clusters of nodes. Two MAC protocol, TDMA and MSAP, were presented to show how a prior knowledge of communication resources and node positions can reduce the overhead in network management and increase communications efficiency. The further development and testing of a formal MAC protocol and data link control protocol is required. Finally, the process of message routing in the spatially dynamic cellular clustering must be addressed. The application scenario that motivated this work is case where multiple AUVs are cooperating in the completion of a common task by sharing locally generated sensor information. The communications required in this scenario require that the immediately adjacent AUVs be able to communicate and share information relevant to the task at hand. As an extension to this scenario, the AUVs are required to report critical information back to a fixed information gateway linking the UAN to on-shore operators. This requires the AUVs to potentially route information across the UAN using multiple hops along the way. Since the geographic position of the information gateway is known, the message can be easily routed using a minimum distance approach of transmitting the message to the nearest neighbor node located in the direct path between the source and the gateway. 
Future Work 


\section{Appendix A}

\section{Spatially Dynamic Cellular Clustering Pseudocode}

\section{A.1 Cell Hopping Algorithm}

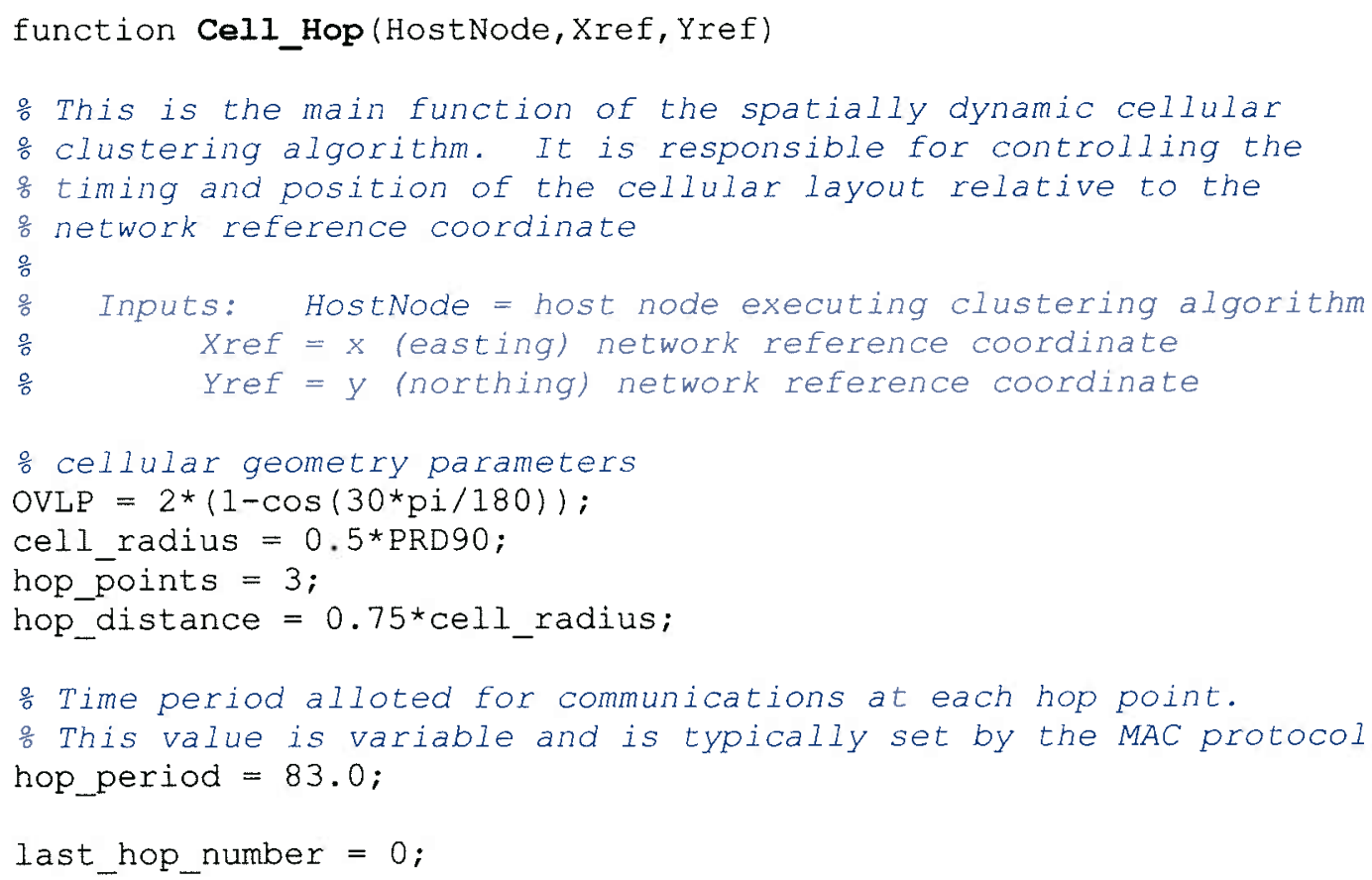




\section{Cell Hopping Algorithm}

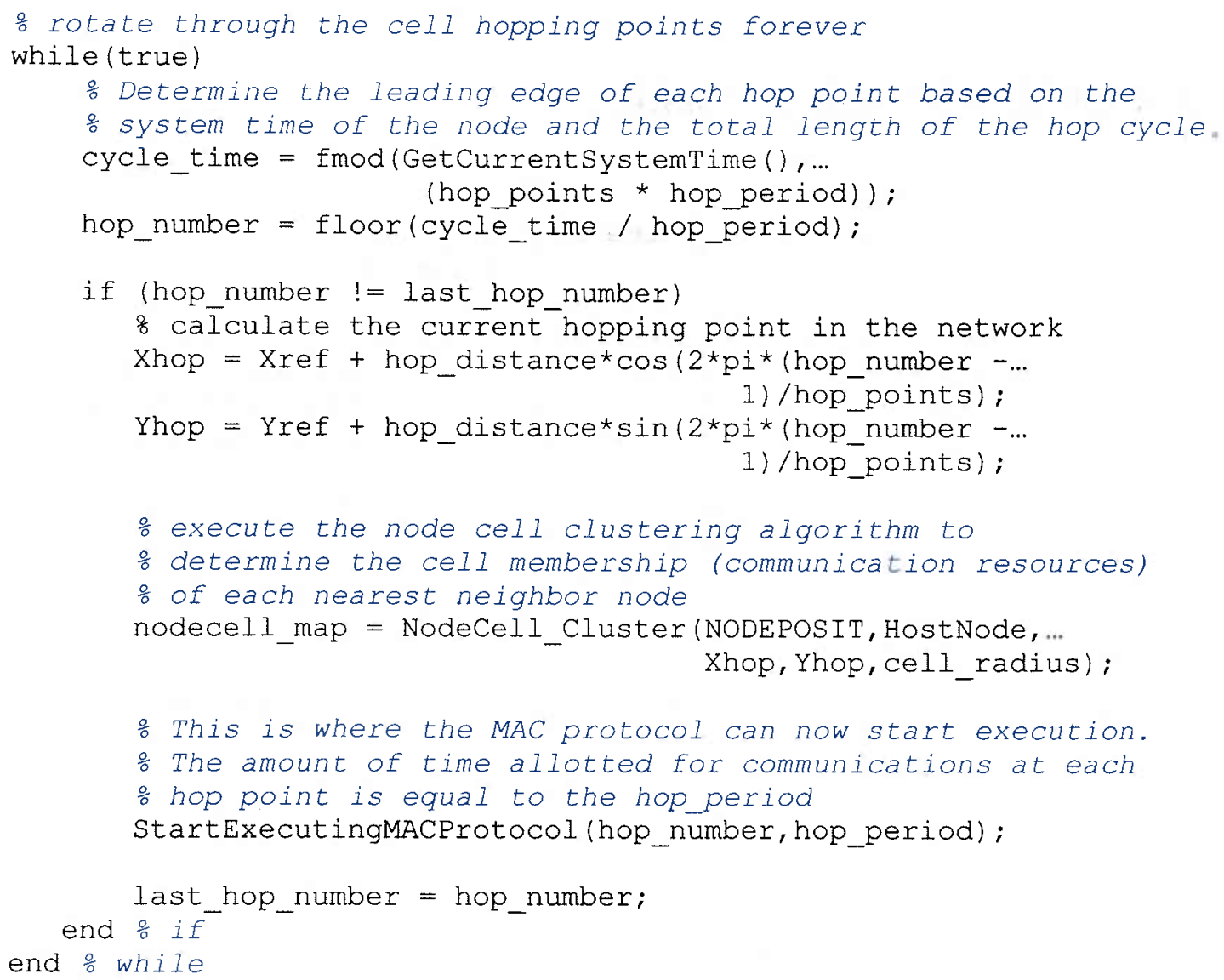




\section{A.2 Cell Membership Algorithm}

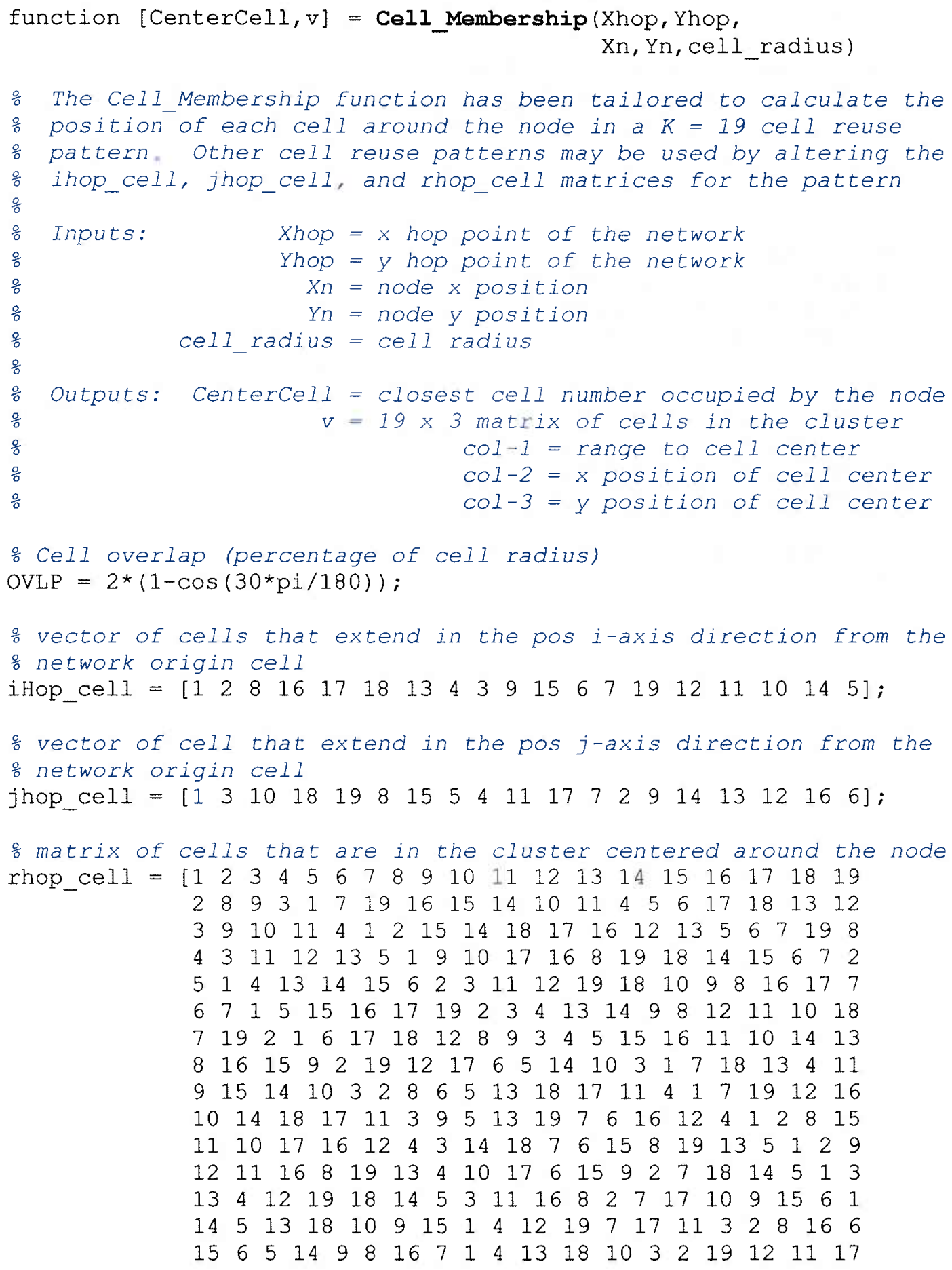


Cell Membership Algorithm

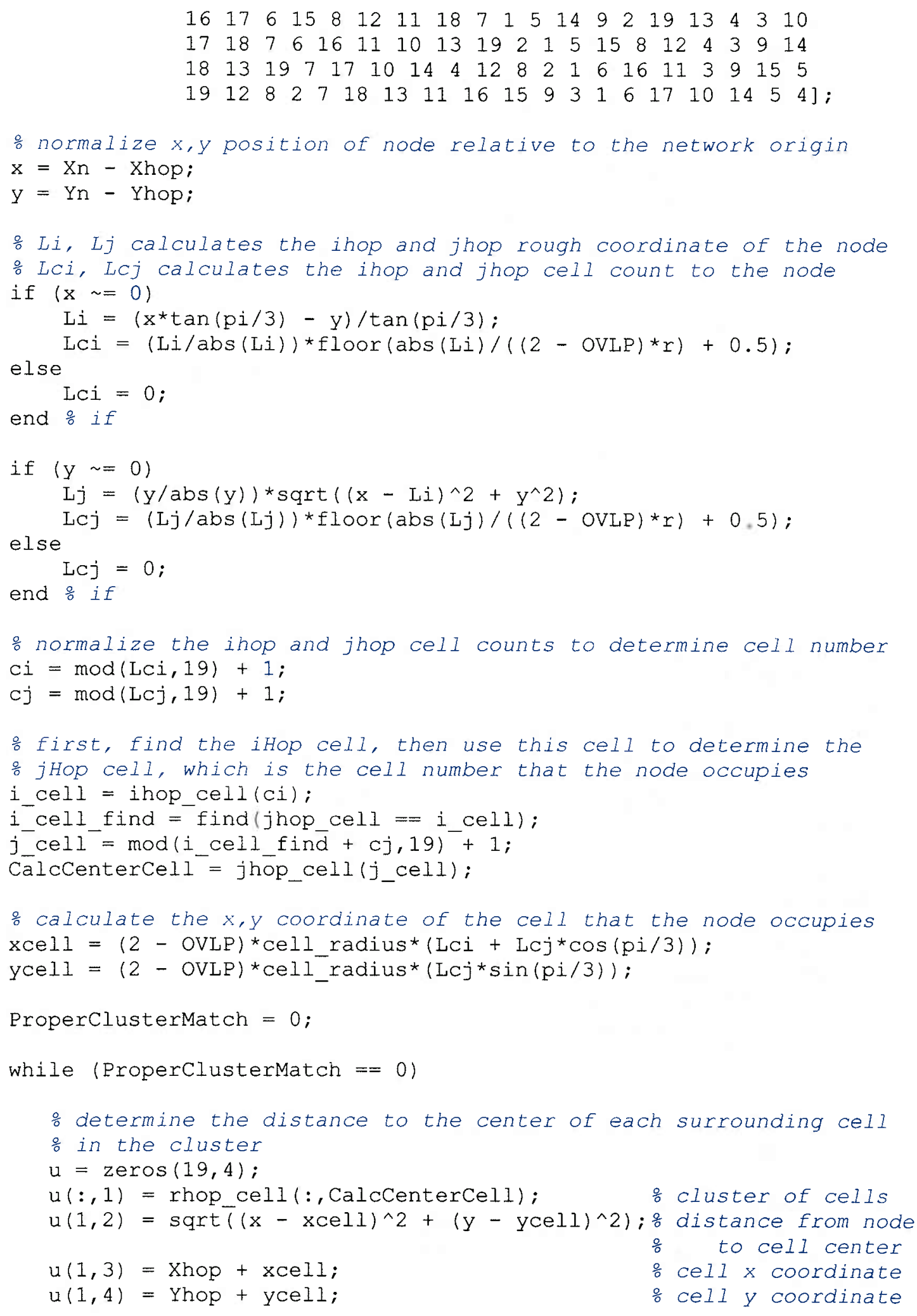




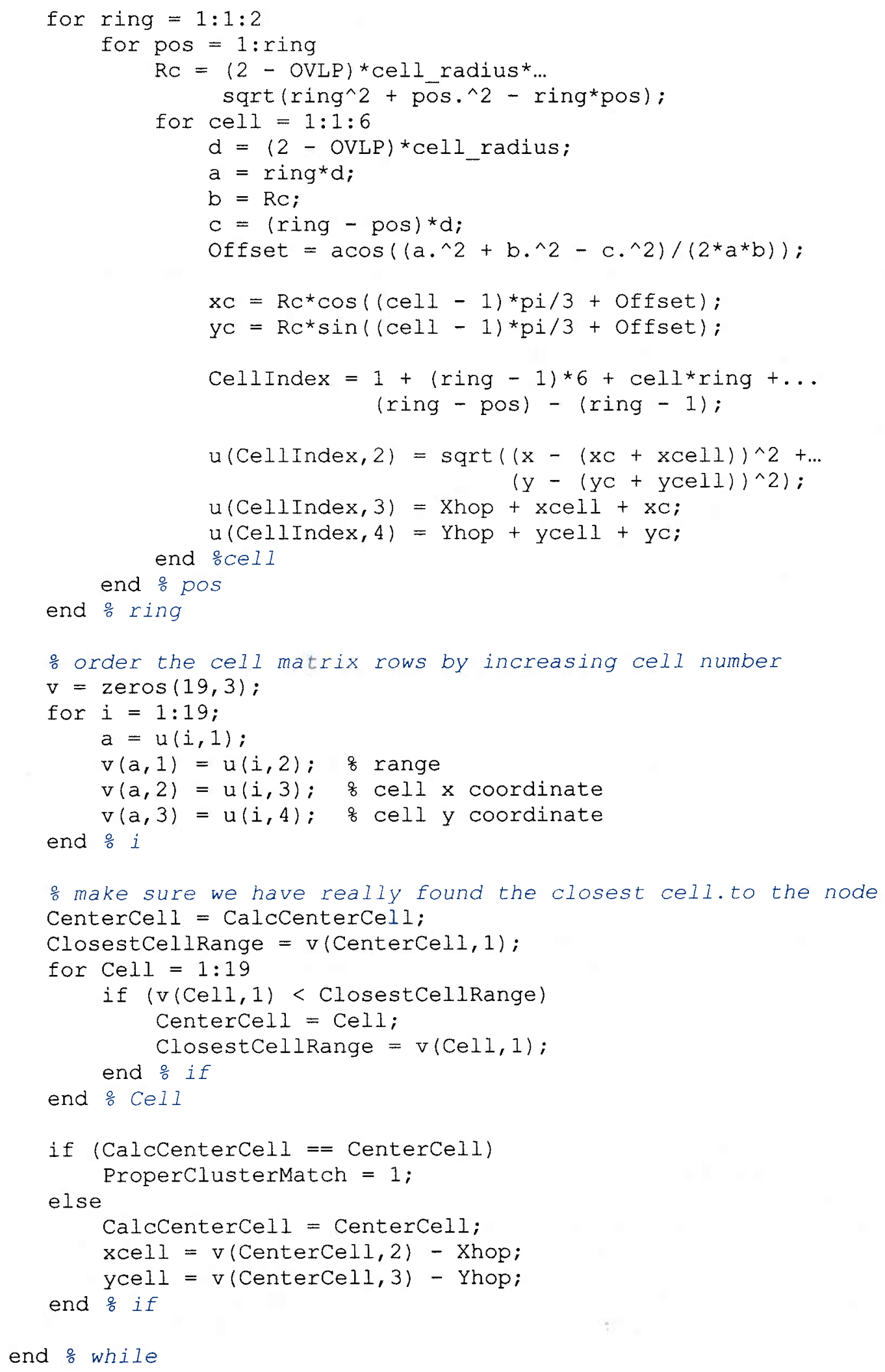


Node Cell Clustering Algorithm

\section{A.3 Node Cell Clustering Algorithm}

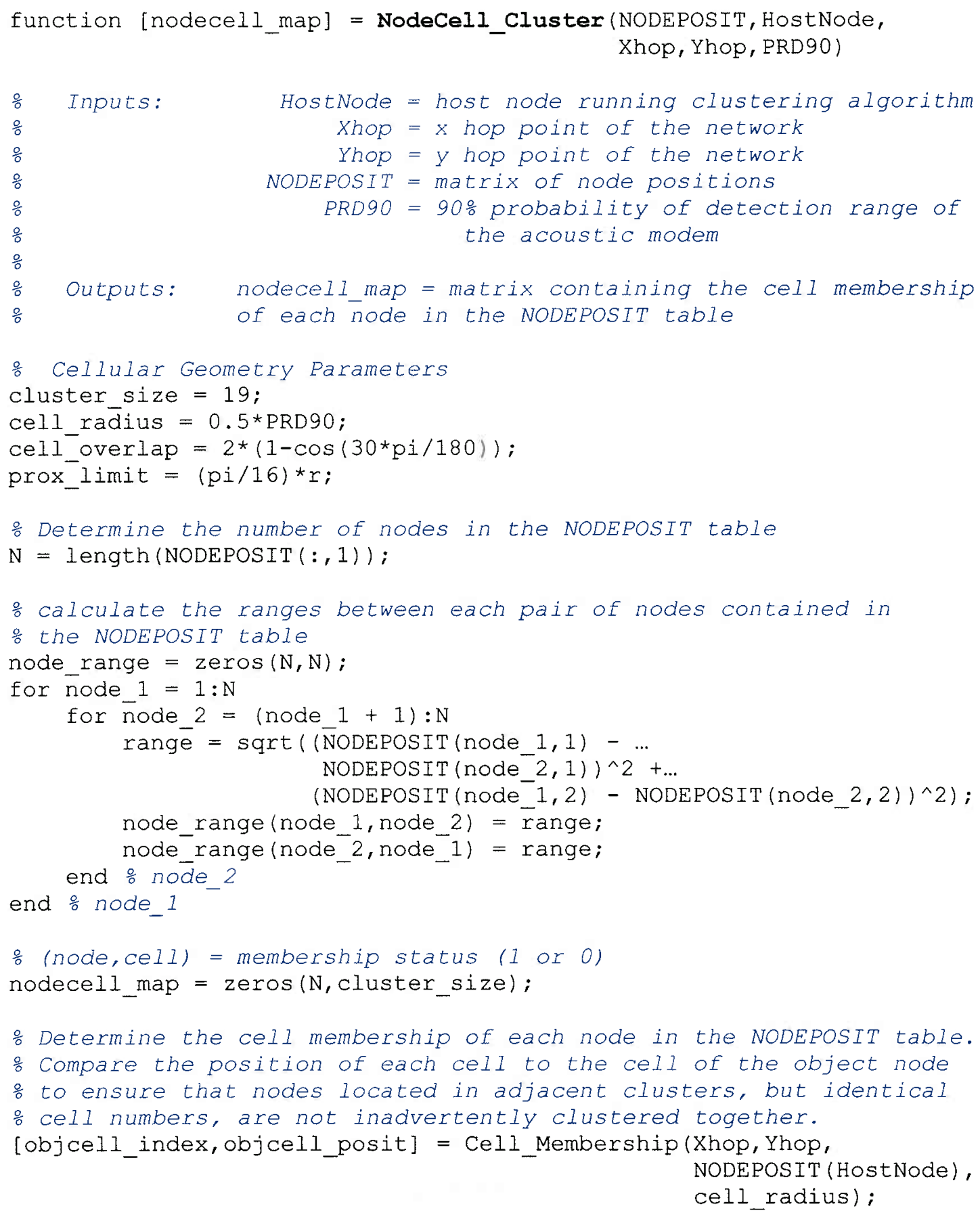




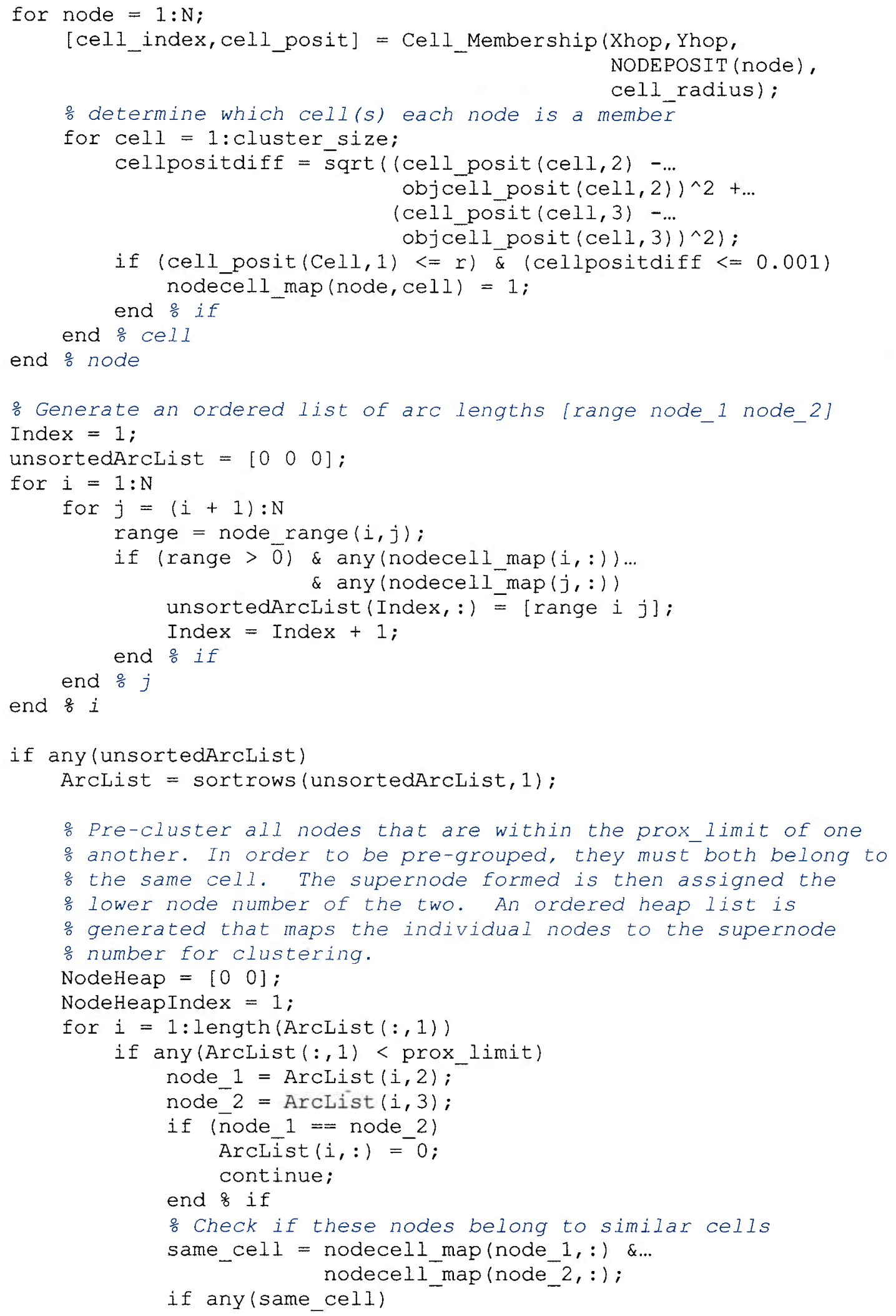




\section{Node Cell Clustering Algorithm}

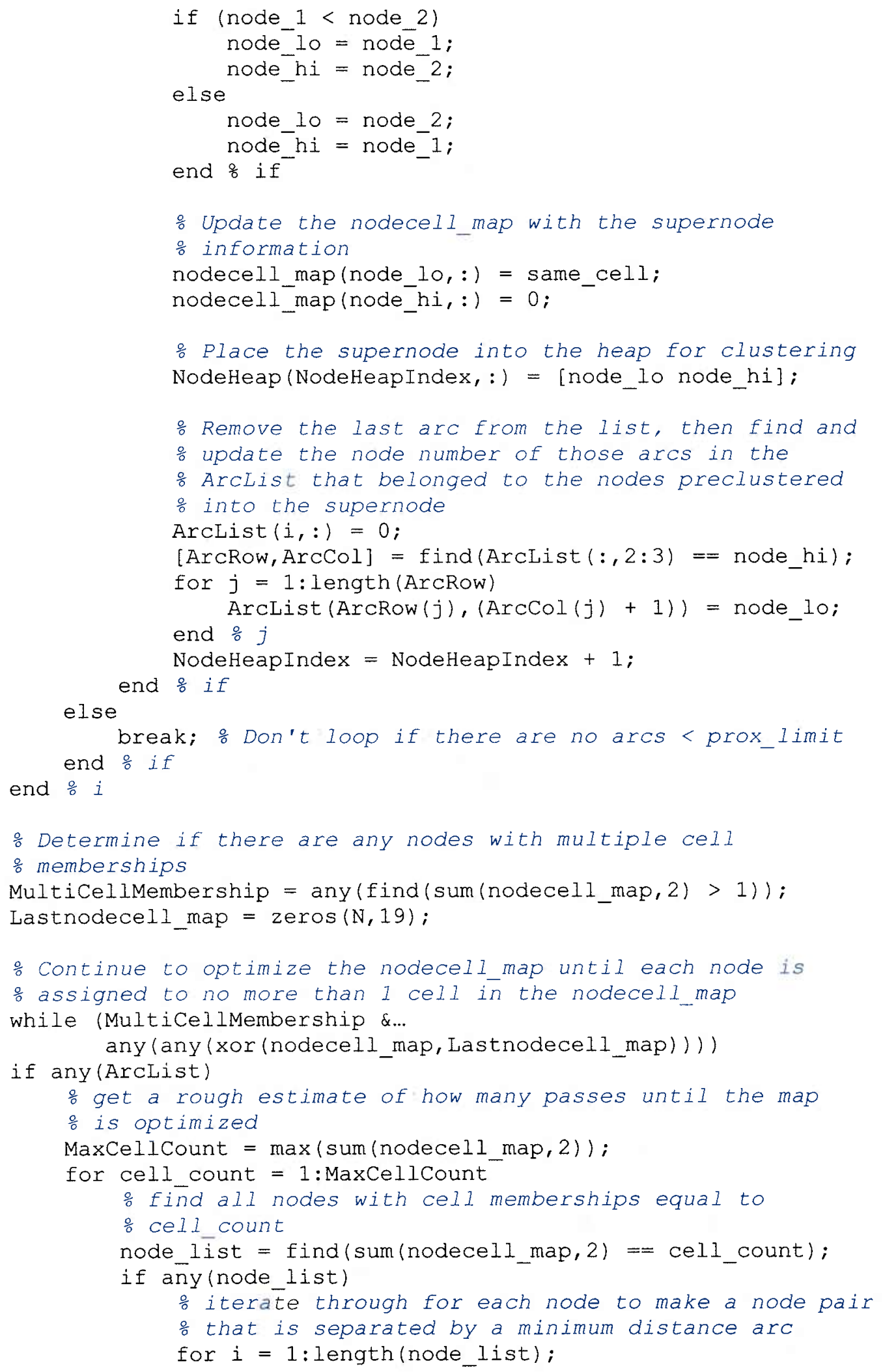




\section{Spatially Dynamic Cellular Clustering Pseudocode}

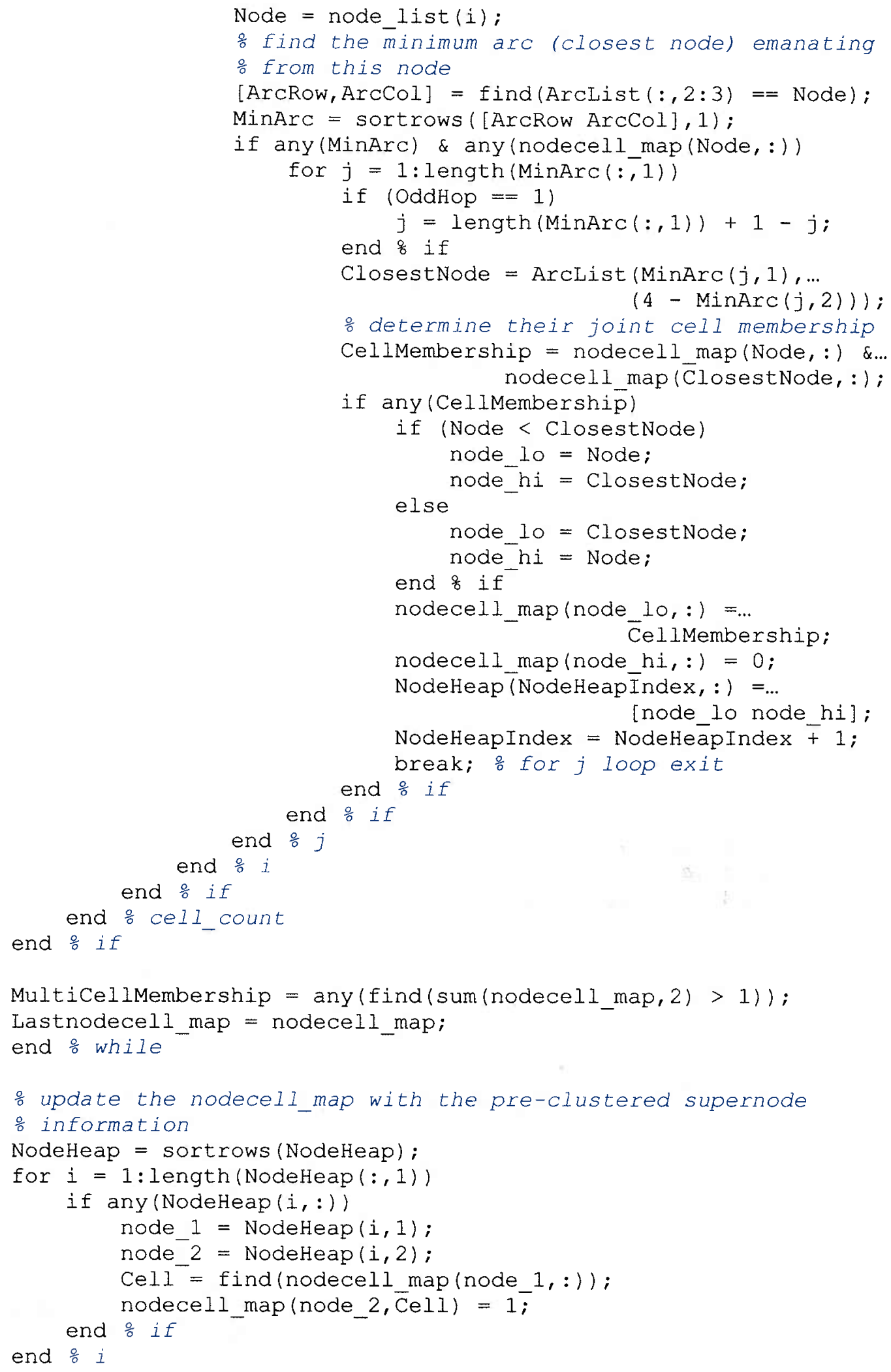


Node Cell Clustering Algorithm

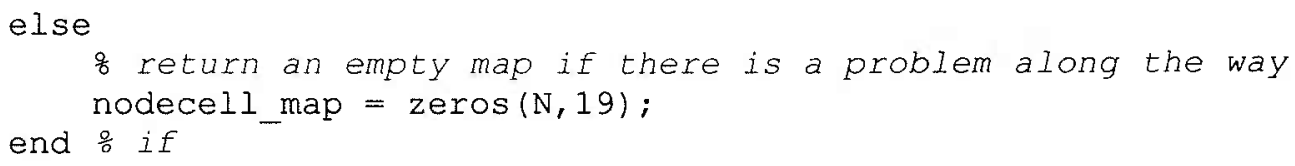




\section{BIBLIOGRAPHY}

[1] F. Blackmon, E. Sozer, M. Stojanovic, and J. Proakis, "Performance Comparison of RAKE and Hypothesis Feedback Direct Sequence Spread Spectrum Techniques for Underwater Communication Applications," presented at MTS/IEEE Oceans 2002 Conference, 2002.

[2] A. B. Baggeroer and D. B. Kilfoyle, "The State of the Art in Underwater Acoustic Telemetry," IEEE Journal of Oceanic Engineering, vol. 25, pp. 4-

[3] E. Sozer, M. Stojanovic, and J. Proakis, "Underwater Acoustic Networks," IEEE Journal of Oceanic Engineering, vol. 25, pp. 72-82, 2000.

[4] A. Alwan, R. Bagrodia, N. Bambos, M. Gerla, L. Kleinrock, J. Short, and J. Villasenor, "Adaptive Mobile Multimedia Networks," IEEE Personal Communications, pp. 34-51, 1996.

[5] D. P. Bertsekas and R. G. Gallager, Data networks, 2nd ed. Englewood Cliffs, N.J.: Prentice-Hall, 1992.

[6] J. H. Gibson and G. G. Xie, "A Network Layer Protocol for UANs to Address Propagation Delay Induced Performance Limitations," presented at MTS/IEEE Oceans 2001 Conference, Honolulu, HI, 2001.

[7] L. Kleinrock and M. Scholl, "Packet Switching in Radio Channels: New Conflict-Free Multiple Access Schemes," IEEE Transactions on Communications, vol. 28, pp. 1015-1029, 1980.

[8] M. Gerla and C. Lin, "Adaptive Clustering for Mobile Wireless Networks," IEEE Journal on Selected Areas in Communications, vol. 15, pp. 1265-1275,

[9] A. McDonald and T. Znati, "A Mobility-Based Framework for Adaptive Clustering in Wireless Ad-Hoc Networks," IEEE Journal on Selected Areas in Communications, vol. 17, pp. 1466-1487, 1999.

[10] J. Rice, "Telesonar Signaling and Seaweb Underwater Wireless Networks," presented at NATO Research \& Technology Agency Symposium on New Information Processing Techniques for Military Systems, 2000.

[11] D. L. Codiga, J. A. Rice, and P. S. Bogden, "Real-time Delivery of Subsurface Coastal Circulation Measurements from Distributed Instruments using Networked Acoustic Modems," presented at MTS/IEEE Oceans 2000 Conference, 2000. 
Node Cell Clustering Algorithm

[12] J. A. Rice, "Seaweb Network for FRONT Oceanogrpahic Sensors," SPAWARS System Center, San Diego, CA December 20012001.

[13] P. S. Bogden and J. A. Rice, "Front-Resolving Observational Network with Telemetry," presented at AGU Ocean Sciences Mtg, San Antonio, TX, 2000.

[14] E. Sozer, M. Stojanovic, and J. Proakis, "Initialization and Routing Optimization for Ad-Hoc Underwater Acoustic Networks," Northeastern University, Boston, MA November 20002000.

[15] M. Stojanovic, L. Freitag, J. Leonard, and P. Newman, "A Network Protocol for Multiple AUV Localization," presented at MTS/IEEE Oceans 2002 Conference, 2002.

[16] M. Stojanovic, J. Proakis, J. A. Rice, and M. Green, "Spread Spectrum Underwater Acoustic Telemetry," presented at MTS/IEEE Oceans 1998 Conference, 1998.

[17] S. Azou, G. Burel, and C. Pistre, "A Chaotic Direct-Sequence SpreadSpectrum System for Underwater Communication," presented at MTS/IEEE Oceans 2002 Conference, 2002.

[18] B. Kim and I. Lu, "Parameter Study of OFDM Underwater Communication System," presented at MTS/IEEE Oceans 2000 Conference and Exhibition, 2000.

[19] M. Stojanovic and L. Freitag, "Multiuser Undersea Acoustic Communications in the Presence of Multipath Propagation," presented at MTS/IEEE Oceans 2001 Conference and Exhibition, 2001.

[20] M. Grund, "WHOI Micro Modem Users Manual." Woods Hole, MA, 2003.

[21] I. Dyer, Fundamentals and Application of Underwater Sound. Cambridge, MA.

[22] W. H. Thorp, "Analytic Description of the Low Frequency Attentuation Coefficient," Journal of the Acoustical Society of America, vol. 42, 1967.

[23] G. V. Frisk, Ocean and seabed acoustics : a theory of wave propagation. Englewood Cliffs, N.J.: Prentice Hall, 1994.

[24] T. S. Rappaport, Wireless communications : principles and practice, 2nd ed. Upper Saddle River, N.J.: Prentice Hall PTR, 2002.

[25] J. G. Proakis, Digital communications. New York: McGraw-Hill, 1983.

[26] H. L. Van Trees, Detection, estimation, and modulation theory. New York: Wiley, 2001. 
[27] M. K. Simon, Spread spectrum communications handbook, Electronic ed. New York: McGraw-Hill, 2002.

[28] J. S. Lee and L. E. Miller, CDMA systems engineering handbook. Boston, Mass.: Artech House, 1998. 